Linköping Studies in Science and Technology

Dissertation No. 2105

\title{
Wireless \\ Bioelectronic \\ Devices Driven by \\ Deep Red Light
}

Marie Jakešová

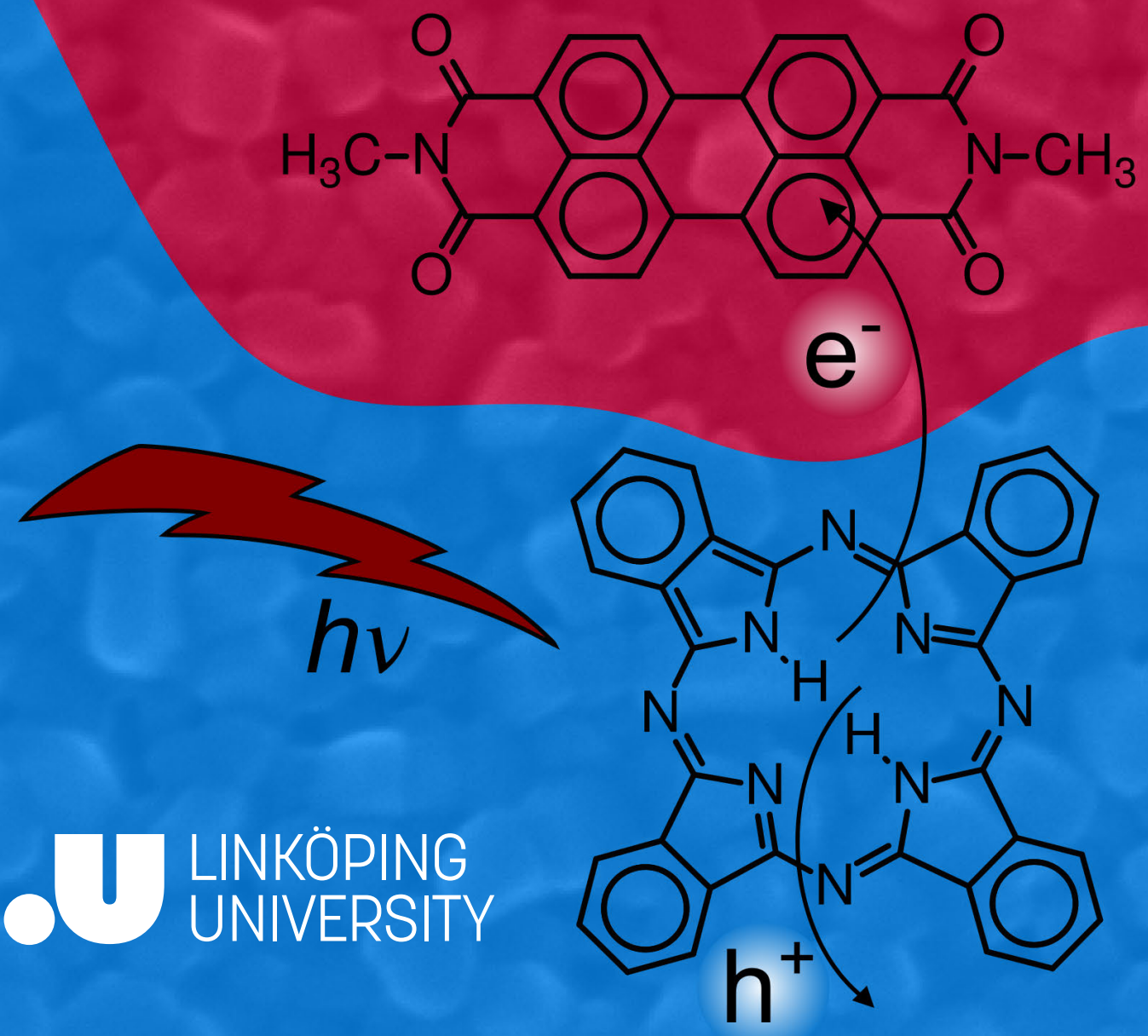



Linköping Studies in Science and Technology, Dissertation No. 2105

\section{Wireless Bioelectronic Devices Driven by Deep Red Light}

Marie Jakešová

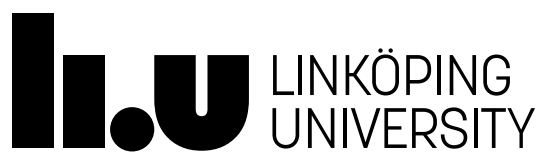

Laboratory of Organic Electronics

Department of Science and Technology

Linköping University, Sweden

Norrköping, 2021 


\section{Description of the cover image:}

The cover image depicts the donor-acceptor bilayer heterojunction based on nanocrystalline small molecule semiconductors used in every paper included in this thesis.

(cc) EY-NC This work is licensed under a Creative Commons AttributionNonCommercial 4.0 International License.

https://creativecommons.org/licenses/by-nc/4.0/

\section{Wireless Bioelectronic Devices Driven by Deep Red Light}

Linköping Studies in Science and Technology, Dissertation No. 2105

(C) Marie Jakešová, 2020 (unless stated otherwise)

During the course of research underlying this thesis, Marie Jakešová was enrolled in Forum Scientium, a multidisciplinary graduate school at Linköping University, Sweden.

Printed in Sweden by LiU-Tryck, Linköping, 2021

Electronic publication: www.ep.liu.se

ISSN 0345-7524

ISBN 978-91-7929-754-1 
Hledej pravdu, slyš pravdu, uč se pravdě, miluj pravdu, mluv pravdu, drž se pravdy, bran̆ pravdu až do smrti

Seek the truth, hear the truth, learn the truth, love the truth, speak the truth, hold the truth and defend the truth until death

- Jan Hus 



\section{Abstract}

The use of electronic devices in medical care is one of the main targets of precision medicine. The field of bioelectronic medicine uses electronic devices to diagnose or treat diseases and disorders in a complementary or alternative way to chemical drugs. It has been more than sixty years since the world's first implantable battery-driven cardiac pacemaker was implanted here in Sweden. Since then, electronic therapies have been implemented for neurological disorders such as Parkinson's disease, epilepsy, sensory and motor function restoration, and many more. However, electronics can also be used for delivery of conventional drugs in a more controlled, localized, and specific fashion.

Therapeutic utility and patient comfort are maximized when the devices are as minimally invasive as possible. The most important milestone in the development of the cardiac stimulator was making it wireless. The early versions of the device required bulky parts to be placed outside of the body with transcutaneous electrical leads to the target site which led to high infection risk and frequent failures. To date, batteries remain the most common way to power implantable electronics. However, their large size and the necessity for replacement surgeries makes the technology relatively invasive. Alternative approaches to wireless power transfer are thus sought after. The most promising technologies are based on electromagnetic, ultrasound, or light-coupling methods.

The aim of this thesis is to utilize tissue-penetrating deep red light for powering implantable devices. The overarching concept is an organic photovoltaic based on small molecule donor-acceptor bilayer junctions, which allows for ultrathin, flexible, minimally-invasive devices. Within this thesis, the photovoltaic device was utilized in two ways. Firstly, the photovoltaics are fabricated to act as an integrated driver for other implantable electronic components: 1) an organic electronic ion pump for acetylcholine delivery; 2) a depth-probe microelectrode stimulation device for epilepsy applications. Secondly, an alternative device, the organic electrolytic photocapacitor, is formed by replacing one of the solid electrodes by an electrolytic contact, thus yielding a minimalistic device acting as a direct photoelectrical stimulator. Within the thesis, the photocapacitive stimulation mechanism is validated by studying voltage-gated ion channels in a frog oocyte model. Next, two lithography- 
based patterning techniques are developed for fabricating these devices with better resolution and on flexible substrates suitable for in vivo operation. Finally, a chronic implant is demonstrated for in vivo sciatic nerve stimulation in rodents. The end result of this thesis is a series of novel device concepts and methods for stimulation of the nervous system using deep red light. 


\section{Populärvetenskaplig sammanfattning}

En av de mest lovande och blomstrande grenarna inom precisionsmedicin är utvecklingen av olika elektroniska implantat som möjliggör medicinsk behandling med hög spatiotemporal upplösning. En del inom detta område är så kallad bioelektronisk medicin vilket inkluderar elektroniska komponenter för att diagnostisera och/eller behandla sjukdomar och störningar på ett kompletterande sätt till kemiska läkemedel. Det har gått mer än sextio år sedan världens första implanterbara batteridrivna hjärtstimulator implanterades här i Sverige. Sedan dess har elektroniska terapier implementerats för neurologiska störningar så som Parkinsons sjukdom, epilepsi, sensorisk och för motorisk funktionsåterställning. Emellertid kan elektronik också användas för att leverera konventionella läkemedel på ett mer kontrollerat, lokalt och specifikt sätt. Användningen underlättas genom att tekniken optimeras för att vara så minimalt invasiv som möjligt. Den viktigaste milstolpen i utvecklingen av hjärtstimulatorn var att göra den helt implanterbar. Detta uppnåddes till dels genom att minska storleken av själva pacemakern, samt att ansluta den till ett miniatyriserat batteri. Hittills är batterier det allra vanligaste sättet att driva implanterbar elektronik, men deras storlek och behovet av ersättningsoperationer gör tekniken relativt invasiv och begränsande. Därför är alternativa metoder att energiförsörja bioelektroniska komponenter eftertraktade och sedfan länge eftersökta. De mest lovande teknikerna är baserade på trådlös kraftöverföring till de implanterade komponenterna, oftast utnyttjas elektromagnetisk överföring, ultraljudeller ljus.

Viss typ av ljus kan tränga igenom hud och ben och på så sätt överföra energi säkert till elektroniska implantat placerade inne i kroppen. Denna avhandling utnyttjar det så kallade vävnadstransparenta fönstret för optisk energiöverföring till bioelektroniska implantat, som då kan användas på ett mer minimalistiskt och icke-invasivt sätt. Med den senaste utvecklingen av lysdioder och laserteknik har användningen av ljus för att driva elektroniska komponenter blivit enklare och mer mångsidig än någonsin. Det är till och med möjligt att använda ljusvåglängder som ligger utanför det synliga spektrat. Sådan teknik möjliggör användning av trådlösa implanterbara apparater utan att orsaka några större obehag för patienter. Neurologisk modulering och elektroniskt kontrollerad läkemedelsleverans är de två bioelektroniska 
begreppen som behandlas i denna avhandling. Genom att göra de elektroniska komponenterna trådlösa gör vi dem inte bara mindre invasiva, och därmed attraktiva för translationell medicin, utan vi öppnar också upp för nya och mer avancerade djurstudier som i framtiden kan främja forskningen inom neurovetenskap. 


\section{Acknowledgements}

Being a $\mathrm{PhD}$ student has been the most exciting and challenging time of my life and I would like to sincerely thank all those, who taught, helped, or influenced me on this path:

My supervisor Magnus Berggren and co-supervisor Daniel T. Simon for giving me the opportunity and the freedom to pursue my scientific interests. Magnus, thank you for always being optimistic and for always seeing the bigger picture. Daniel, thank you for your support, your editing skills and your tips on presentation technique.

My co-supervisor Fredrik Elinder for giving me the opportunity to do electrophysiology in his laboratory, for deep, challenging scientific discussions, and for always being ready to help. I only wish I had the chance to spend more time at your institute.

The former and current members of my two home bases - the Organic Bioelectronics group, and the Organic Nanocrystals group - for providing a stimulating environment to learn in. I would especially like to thank the "bioelectricians" Theresia Arbring Sjöström, David Poxson, Erik Gabrielsson and Iwona Bernacka-Wójcik for introducing me to fabrication and characterization of organic electronic ion pumps. My sincerest gratitude goes to the "nanocrystals" Eric D. Glowacki and Vedran Đerek, my mentors and lab superheroes, who taught me almost everything else in the cleanroom, always challenged me, and never let me drink coffee without a proper scientific discussion. Ludovico Migliaccio for being my partner in crime in most of the photocapacitor fabrications, my tool co-responsible, and the best guitar player I know. Maciej Gryszel for knowing everything about chemistry, being the best Forum Scientium buddy, and for letting me play with the cutest puppy in Norrköping. Malin Silverå Ejneby for teaching me all about voltageclamp, for being the best at data analysis, and for believing in the photocapacitor idea enough to join the group in Norrköping. Igor Sagalianov "the theoretician" for talking to us even though we babble about cleanroom all the time, and for being a great gym coach. Mary J. Donahue for being a true photolithography queen always keen to help, the best co-traveler both for experiments and for camping trips, and for always being ready um Vier. Mary's husband Adam Williamson for 
moving Mary to Sweden, being the most enthusiastic applied neuroscientist, and liking Marge as much as I do.

All my external colleagues and co-authors for amazing collaborations. Special thanks go to David Rand, Ieva Vèbraité, and Yael Hanein at Tel Aviv University; Jennifer Gelinas, Dion Khodagholy and Jose Ferrero Lopez at Columbia University; April Caravaca and Peder Olofsson at Karolinska Institute; Florian Hartmann and Martin Kaltenbrunner at Johannes Kepler University; and Tony Schmidt, Linda Waldherr, and Rainer Schindl at Medical University of Graz.

Lasse Gustavsson, Thomas Karlsson and Meysam Karami Rad for being the best lab crew, for helping me solve many problems at the speed of light, and always being ready for a joke. Big thank you goes also to Anna Malmström for managing the lab space and us, the unruly users. Gustav Knutsson for access to the laser photoplotter and help with mask production.

Other colleagues and friends at the Laboratory of Organic Electronics for creating a welcoming environment. My special thanks go to Sämi for commenting on the thesis draft and being the social activities guru. Nadia for always being so cheerful and for organizing fun events. Chiara for being a great conference buddy and gym enthusiast. Mehmet for bringing a lion-like Turkish spirit. Josefin and David for great trips and good tips in the beginning. Dennis, Eva, Donghyun, Jee Woong, Xeno, Ulrika, Jennifer and Yiannis, for many nice evenings.

Forum Scientium and Stefan Klintström for organizing great summer schools and interesting seminars.

Finally, I would like to thank my dear family and friends for their love, support and for always being there for me.

Děkuji 


\section{List of included publications}

Paper I

Optoelectronic control of single cells using organic photocapacitors

Marie Jakešová, Malin Silverå Ejneby, Vedran Đerek, Tony Schmidt, Maciej Gryszel, Johan Brask, Rainer Schindl, Daniel T. Simon, Magnus Berggren, Fredrik Elinder, Eric D. Głowacki

Science Advances, 2019, 5(4), eaav5265.

Contributions: all device design, fabrication and characterization, most of the cell experiments and data analysis, the first draft

Paper II

Micropatterning of organic electronic materials using a facile aqueous photolithographic process

Vedran Đerek, Marie Jakešová, Magnus Berggren, Daniel T. Simon, Eric D. Głowacki

AIP Advances, 2018, 8(10), 105116.

Contributions: contributions to device fabrication and characterization, contribution to the first draft

Paper III

A chronic photocapacitor implant for noninvasive neurostimulation with deep red light

Malin Silverå Ejneby ${ }^{\dagger}$, Marie Jakešová ${ }^{\prime}$, Jose J. Ferrero ${ }^{\dagger}$, Ludovico Migliaccio $^{\dagger}$, Zifang Zhao, Magnus Berggren, Dion Khodagholy, Vedran Đerek, Jennifer Gelinas, Eric D. Głowacki

Manuscript in revision

Contributions: chronic device design, fabrication and characterization, contribution to the chronic experiments and data analysis, contribution to the first draft

$\dagger$ Shared first authorship 
Paper IV

\section{Ultrathin organic photovoltaic microstimulators}

Marie Jakešová, Magnus Berggren, Daniel T. Simon, Jennifer Gelinas, Dion Khodagholy, Eric D. Głowacki

Manuscript in preparation

Contributions: device design, all fabrication and characterization, the first draft

Paper V

Wireless organic electronic ion pumps driven by photovoltaics

Marie Jakešová, Theresia Arbring Sjöström, Vedran Đerek, David J. Poxson, Magnus Berggren, Eric D. Głowacki, Daniel T. Simon

Npj Flexible Electronics, 2019, 3(14), 1-6.

Contributions: the project idea, device design, fabrication and characterization, the first draft and final manuscript editing, corresponding author on the manuscript 


\section{Related publications not included in the thesis}

Extracellular photovoltage clamp using conducting polymermodified organic photocapacitors

Malin Silverå Ejneby, Ludovico Migliaccio, Mindaugas Gicevičius, Vedran Đerek, Marie Jakešová, Fredrik Elinder, Eric D. Głowacki

Advanced Materials Technologies, 2020, 5(3), 1900860.

Targeted chemotherapy of glioblastoma spheroids with capillary fiber organic electronic ion pumps

Linda Waldherr, Maria Seitanidou, Marie Jakešová, Verena Handl, Sophie. Honeder, Marta Nowakowska, Tamara Tomin, Tony Schmidt, Joachim Distl, Romana Schober, Ruth Birner-Grünberger, Gord von Campe, Beate Rinner, Martin Asslaber, Nassim Ghaffari Tabrizi-Wizsy, Silke Patz, Daniel T. Simon, Rainer Schindl

Submitted

Parygami: Scalable microfabrication of folded parylene for stretchable electronics

Florian Hartmann, Marie Jakešová, Guoyong Mao, Marta Nikić, Martin Kaltenbrunner, Vedran Đerek, Eric D. Głowacki

Submitted 



\section{Table of Contents}

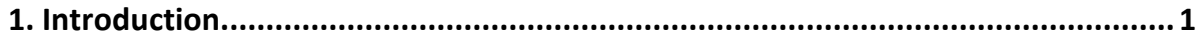

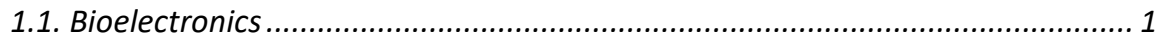

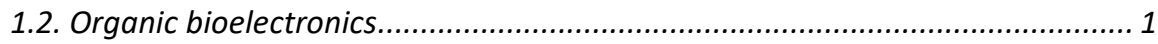

1.3. Implantable electronics............................................................................ 2

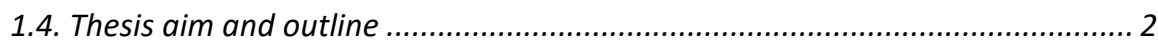

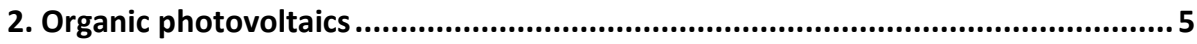

3. Electrical properties of biological systems ..................................................9

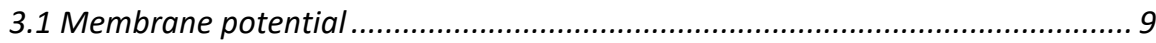

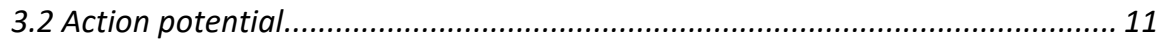

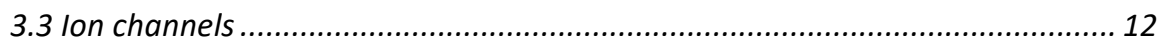

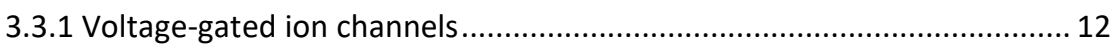

3.4 The in vitro model - Xenopus laevis oocyte .................................................... 13

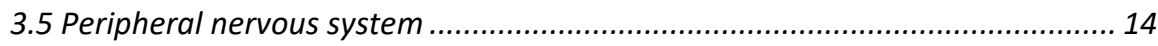

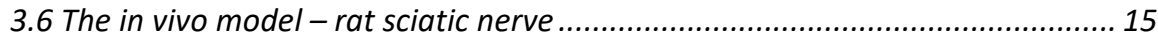

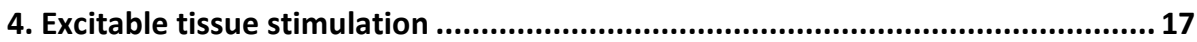

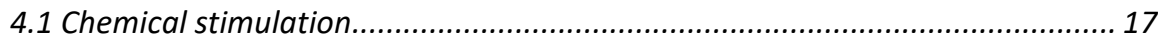

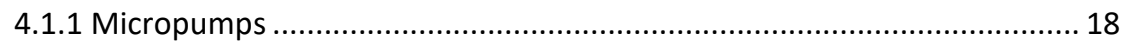

4.1.2 Conducting polymer electrostatic actuators ........................................ 18

4.1.3 Electrophoretic delivery .................................................................. 19

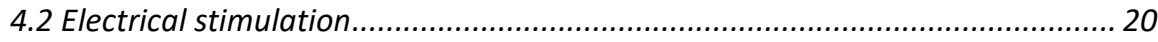

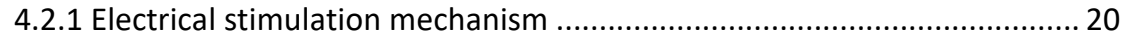

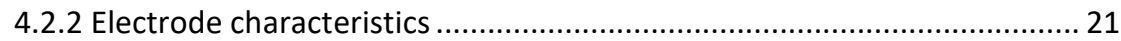

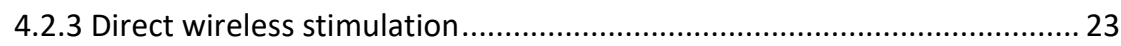

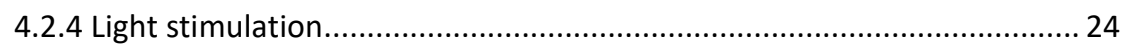

4.2.5 Organic electrolytic photocapacitors .................................................. 27

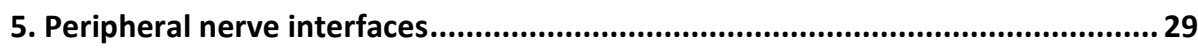

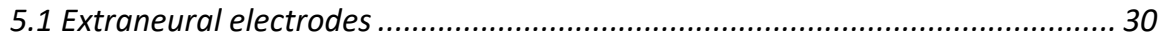

5.2 Wireless powering of biomedical devices ...................................................... 32

5.2.1 External power transfer systems........................................................... 32

5.2.2 Internal power transfer systems ..................................................... 35 


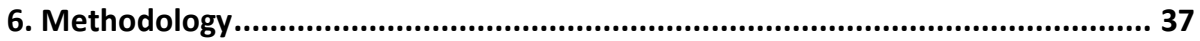

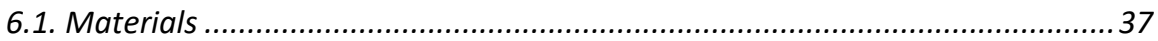

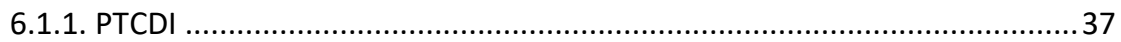

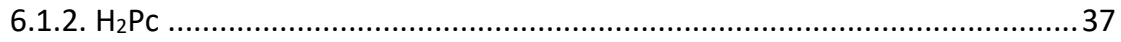

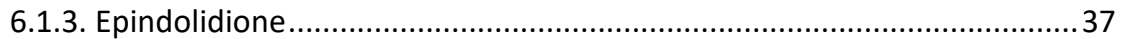

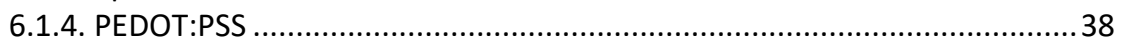

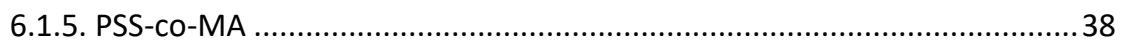

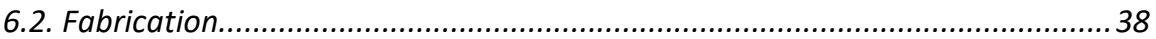

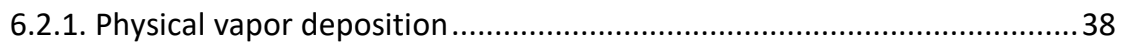

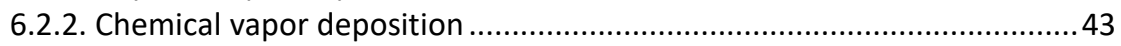

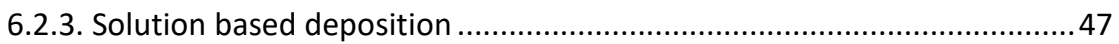

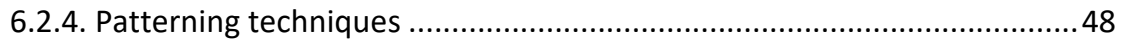

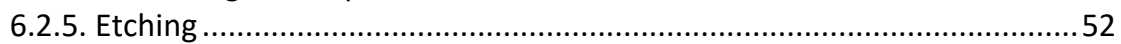

6.2.6. Fabrication schemes for Papers III, IV, and V ......................................5 54

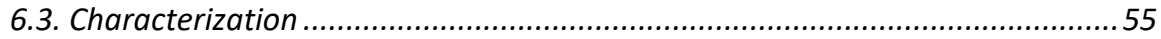

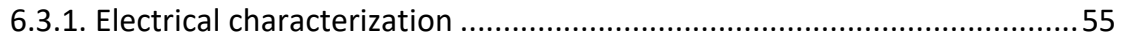

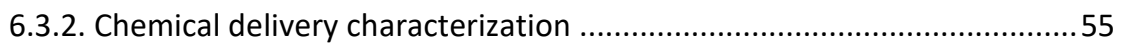

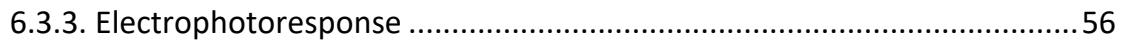

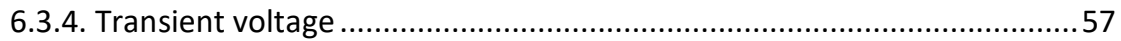

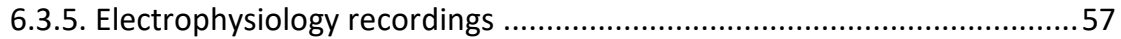

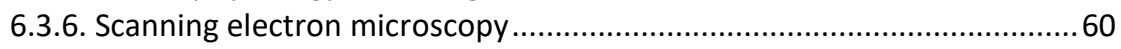

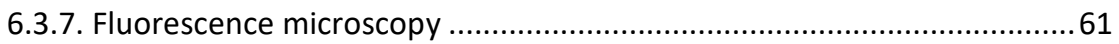

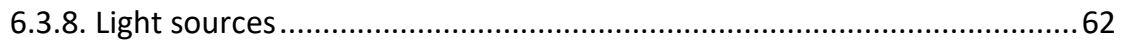

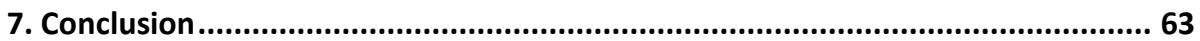

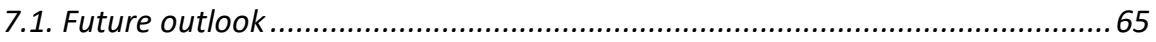

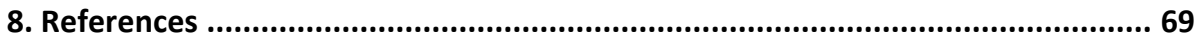




\section{Introduction}

\subsection{Bioelectronics}

Bioelectronics is a scientific and engineering discipline utilizing electronic devices and systems in biomedical applications. The objective of the field is the design and development of devices and interfaces for basic biological research, biotechnology, and medicine. The range of applications spans from electronic biosensors for agent sensing and monitoring, to controllable drug delivery, to electronic implants for neurological, neuropsychiatric, and cardiac diseases.

\subsection{Organic bioelectronics}

Organic electronics leverages the ability of certain carbon-based materials to act as conductors or semiconductors. The field accelerated with the discovery of electrical conductivity in halogen-vapour doped trans-polyacetylene. ${ }^{1}$ Organic materials possessing extended conjugated $\pi$-bond systems show several crucial electronic properties in combination with chemical features making them interesting to explore at the interface between technology and biology. The two main categories of organic electronic materials are small molecule semiconductors and conducting polymers. Organic electronic materials gained vast interest due to the ease of altering the energetics by changing the chemical structure, the possibility of low-cost and low-temperature processing on cheap and flexible substrates, and superior mechanical properties. By far, the most developed and wide-spread device of organic electronics is the organic light emitting diode (OLED) ${ }^{2}$, which has been deployed in displays and lighting applications. Major interest is also invested into organic photovoltaics $(\mathrm{OPV})^{3}$ and various types of organic transistors, which are especially well-suited for biological sensing. ${ }^{4}$ Organic electronic materials are also excellent candidates for use in bioelectronics, because their coupled physical and chemical properties are easily tuned by molecular design. They present options to make electronic devices softer, thinner, and more biocompatible, but also open completely new device concept possibilities. ${ }^{4}$ 


\subsection{Implantable electronics}

One of the most influential steps in the development of implantable electronics was detaching them from external components to make them fully wireless. The first remote operation approach was based on batteries and this remains the most widespread solution to-date. ${ }^{5}$ Depending on the device power consumption, batteries can power implants for 2-10 years. After that, the patient has to undergo a replacement surgery. The battery size sometimes requires implantation in an established body cavity far from the stimulation target, thereby requiring lead cables that are prone to failure. Furthermore, the electrolytes used in batteries are toxic and can cause poisoning in case of leakage. All these potential disadvantages drive interest in alternative power transfer technology. The most explored systems are based on electromagnetic, ultrasonic or optical power transfer. ${ }^{5}$ Light offers high spatial and temporal resolution for studies in vitro. In addition, the partial transparency of biological tissue to red and near-infrared light makes applications in vivo plausible. Due to the high level of scattering in the tissue, the possible implantation depth is relatively shallow $(\leq 2 \mathrm{~cm})$ and light coupling is thus suited especially for subdermal applications. Typically, inorganic photovoltaics (PV) have been explored for in vivo powering of devices due to their high power conversion efficiencies. ${ }^{6}$ However, inorganic PVs are usually at least a few $\mu \mathrm{m}$ thick, because of their low absorption coefficient. On the contrary, organic semiconductors offer high absorption coefficients, making devices on the order of 100-200 nm possible. Therefore, organic electronic materials are ideal candidates for ultra-flexible and minimallyinvasive implant applications to improve patient comfort and minimize tissue foreign body reaction.

\subsection{Thesis aim and outline}

The studies presented in this thesis were focused on exploring the possibilities of a simple donor-acceptor bilayer heterojunction based on nanocrystalline organic small molecule semiconductors: metal-free phthalocyanine $\left(\mathrm{H}_{2} \mathrm{Pc}, \quad \mathrm{p}\right.$-type $)$ and $N, N^{\prime}$-dimethyl-3,4,9,10perylenetetracarboxylic diimide (PTCDI, n-type) in terms of the in vitro and in vivo bioelectronics paradigm. 
Papers I, II and III describe the heterojunction in the form of an organic electrolytic photocapacitor (OEPC). ${ }^{7}$ OEPCs are simple photostimulation devices based on a semitransparent bottom conductor with a photopixel bilayer on top. These devices give rise to voltage transients in electrolytes when irradiated with light. Paper I elucidates the OEPC stimulation mechanism using a single cell electrophysiology model, providing evidence for the photocapacitive mode of action on voltage-gated ion channels. Within Paper II we develop a new photolithographic method for patterning of organic electronic materials based on dual exposure of a positive-tone photoresist. With that method, the OEPC device resolution and precision are improved compared to devices made using a conventional fabrication method based on shadow masking. In Paper III, the first in vivo demonstration of the OEPC stimulation is presented. The OEPC is here used to chronically stimulate the sciatic nerve in a rat model. To achieve that, the fabrication is transferred to an ultrathin substrate, another patterning method for the organic pixels is developed, and an anchoring solution for the implant is designed.

Papers IV and V demonstrate the heterojunction in the form of organic photovoltaic cells to power and operate other implantable devices. In Paper IV a research project is reported in which the photovoltaic driver is connected to a pair of stimulation microelectrodes to create a microstimulator. The aim is to increase the stimulation specificity compared to the OEPC by concentrating the photogenerated charge on the microelectrodes. In Paper V, the use of the photovoltaic driver in combination with an electrophoretic drug delivery device, the organic electronic ion pump (OEIP $)^{8}$ is reported. It shows actuation of proton and neurotransmitter (acetylcholine) delivery based on the projected light intensity.

The thesis aims to provide the reader with the theoretical and practical background to put the included papers into perspective. Chapter 2 introduces the concept of organic photovoltaics, the main building-block of all the devices developed within the thesis. Chapter 3 describes the electrical properties of biological systems, with emphasis on the in vitro model of Xenopus laevis oocytes utilized in Paper I and the in vivo model of the rat sciatic nerve studied in Paper III. The approaches to tissue stimulation are laid out in Chapter 4. Implantable devices for peripheral nerve stimulation are discussed in Chapter 5, along with methods used to wirelessly power them. Chapter 6 covers the materials, fabrication 
processes, and characterization methods employed. Finally, Chapter 7 discusses the projects in a chronological order in attempt to provide the thought processes that eventually shaped my $\mathrm{PhD}$ path. Further, it describes the challenges and opportunities left for the years to come. 


\section{Organic photovoltaics}

Photovoltaics are semiconductor devices that convert light into electrical power, traditionally used for solar energy conversion. Within this thesis, organic photovoltaic cells, or devices directly derived from them, are used as wireless power sources for various biomedical applications. This chapter provides an introduction into the operation of organic photovoltaics to serve as background for the subsequent chapters.

Conventional materials for photovoltaic devices are based on inorganic semiconductors, such as silicon and gallium arsenide. The development of polycrystalline silicon solar cells has recently dominated the market due to lowered production costs and reasonable efficiency. However, since the rise of organic electronics, organic materials have been studied deeply for the application with hope for large-scale / low-cost production. The advantage of organic materials is their high absorption coefficient $\left(\sim 10^{5} \mathrm{~cm}^{-1}\right)$ thus allowing absorber thickness in the order of 10-100 nm. Another advantage of organics is the possibility to tune the energetic and chemical properties by synthesis. One of the largest drawbacks of organics as photovoltaics lies in their low dielectric constant which results in high exciton binding energy. ${ }^{9,10}$ To overcome this problem, Tang developed evaporated organic donor-acceptor heterojunction photovoltaic cells. This important achievement was reported in 1986. In this work, the heterojunction provides the necessary built-in field to overcome the exciton binding energy, which results in efficient charge generation and separation. The first cell based on a $\mathrm{p}-\mathrm{n}$ junction included the combination of copper phthalocyanine and a perylene diimide derivative, and showed $0.95 \%$ power conversion efficiency. ${ }^{3}$ The two main classes of organic PVs are based on small molecule vacuum evaporated planar heterojunctions and polymer bulk heterojunction (BHJ) blends processed from solution. The concept of BHJ (bicontinuous and interpenetrating donor-acceptor phases) was introduced to overcome the small exciton diffusion lengths present in organics due to their inherent disorder and presence of defects. Depending on the material, the exciton diffusion length is on the order of tens of nm. Bulk heterojunctions with extensive percolating paths allow the excitons to be separated and the charge carriers to be collected by the electrodes at high yields. In solution-processed solar cells, the BHJ is produced by the phase 
separation of the donor and acceptor molecules following solvent evaporation. Using this method, power conversion efficiency of $17.4 \%$ has recently been certified. ${ }^{11}$ Creating bulk heterojunctions in physical vapor deposited OPVs is more challenging, but can lead to a $100 \%$ increase in the power conversion efficiency relative to the planar design. ${ }^{12}$ However, the most common approach to enhance the power conversion efficiency in thermally evaporated OPVs is vertical stacking of several bilayer heterojunctions in series. ${ }^{10,13}$ Using this technique, Heliatek GmbH reports $13.2 \%$ power conversion efficiency. ${ }^{14}$

The architectures of planar heterojunction and bulk heterojunction OPVs are shown in Figure 2.1a. The performance of an OPV is typically represented by three parameters of the cell $\mathrm{J}(V)$ curve (Figure $2.1 \mathrm{~b}$ ), short circuit current density $\left(J_{S C}\right.$, the photocurrent density under zero bias), open circuit potential $\left(V_{O C}\right)$ and the fill factor $(F F)$ i.e. the ratio of the power generated at the maximum power point $\left(M_{P P}\right)$ and the theoretical power at $\mathrm{V}_{\mathrm{OC}} \cdot \mathrm{I}_{\mathrm{SC}}$. The product of the three parameters divided by the incoming power $\left(P_{I}\right.$, in solar cell literature typically $\left.100 \mathrm{~mW} \cdot \mathrm{cm}^{-2}\right)$ is defined as the power conversion efficiency $\left(\eta_{P}\right)$ and can be calculated according to the equation below.

$$
\eta_{P}=\frac{J_{S C} \cdot V_{O C} \cdot F F}{P_{I}}
$$

a

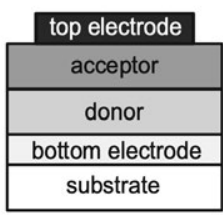

top electrode

electron trans. layer
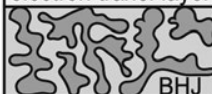

hole trans. layer

bottom electrode

substrate

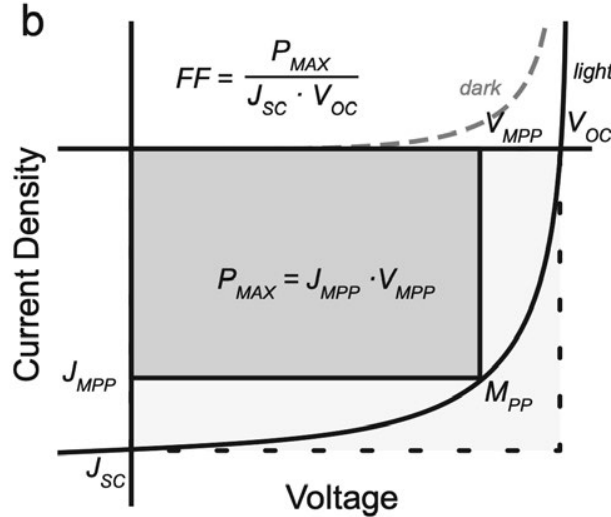

Voltage

Figure 2.1: a) Typical OPV structures: bilayer (top) and bulk heterojunction (bottom). b) A representative OPV $\mathrm{J}(V)$ curve in dark and light conditions, all the important parameters for power conversion efficiency calculation are marked. 
These three parameters have to be optimized to yield high efficiency devices. The photovoltage of an OPV is intrinsically dictated by the energy levels of the donor and acceptor molecules. The maximum voltage the cell can deliver is given by the difference between the donor ionization potential and the acceptor electron affinity and it is very sensitive to material impurities. The mechanism of photocurrent generation in organic photovoltaics is illustrated in Figure 2.2. In the first step, the active molecule absorbs a photon thereby creating a bound electron-hole pair i.e. an exciton. The exciton diffuses to the donor/ acceptor heterojunction where it separates into an electron and a hole due to the energetic offset between the two materials. Finally, the charges are extracted via both drift and diffusion by the collecting electrodes. The value of photocurrent depends on the product of efficiency of each of the four steps. ${ }^{10}$ The fill factor is a figure of merit that quantifies the loss mechanisms in the solar cell and it can be maximized by ensuring small series resistance and high shunt (vertical) resistance of the device.

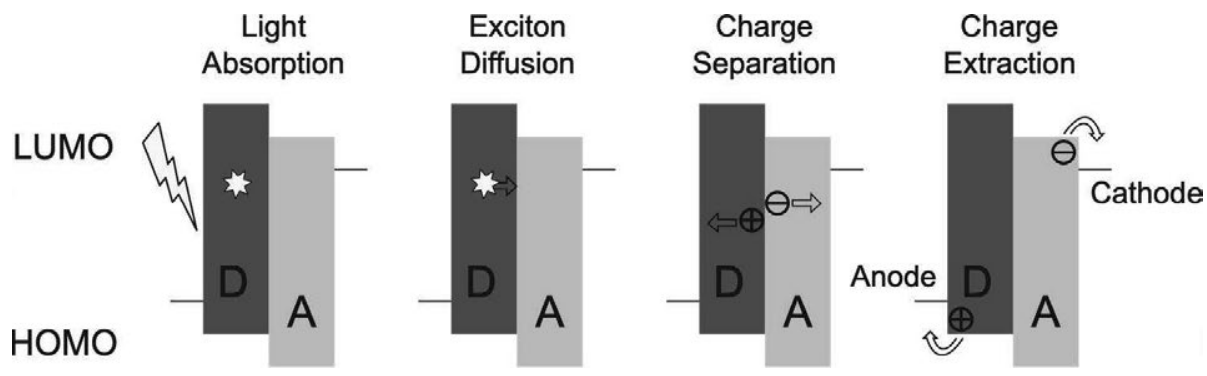

Figure 2.2: Schematic of the OPV charge generation mechanism under open circuit condition. $\mathrm{D}=$ donor molecule, $\mathrm{A}=$ acceptor molecule.

The use of organic photovoltaics in medicine is attractive due to the material's mechanical properties, enabling ultra-thin, flexible devices with the absorption spectrum tuned for the specific application. The devices used in this thesis are solely based on planar $p-n$ heterojunction using materials reported by Hiramoto ${ }^{13}$, because the evaporated small molecule heterojunctions have shown promising operation and stability in biologically relevant environments. ${ }^{7}$ 



\section{Electrical properties of biological systems}

A cell is the main building block of all living organisms. The cell cytoplasm is separated from the outside world by a membrane built of phospholipid bilayer impermeable to most substances. To facilitate communication and homeostasis, there are proteins imbedded in the plasma membrane such as ion channels, receptors, and transporters. Thus, the cell membrane can be seen as a capacitor in parallel with a resistor due to its dielectric nature and selective permeabilities. The electrical properties of cells are determined by the different concentrations of ions inside and outside of the cell, which give rise to the membrane potential, defined as the difference in electrical potential between the cell interior and exterior. This potential difference is maintained by ion pumps, which move ions against their concentration gradient at the expense of metabolic energy. Some cells use the presence of a membrane potential and its rapid changes to exert their function in the form of action potentials. These cells are generally called excitable and include neural, muscle, and some endocrine cells. The excitability is provided by the membrane's ability to undergo changes in specific ion's permeability, which leads to a rapid change in the membrane potential thus triggering an action potential. The permeability is caused by the opening of ion channels, transmembrane pore-forming proteins which allow passive diffusion of specific ions through the membrane down their concentration gradient. This chapter covers the basis of cellular excitability and describes the in vitro and in vivo validation models used within this thesis.

\subsection{Membrane potential}

The most important players in the membrane excitability are $\mathrm{K}^{+}, \mathrm{Na}^{+}$, $\mathrm{Ca}^{2+}$, and $\mathrm{Cl}^{-}$, to which the membrane is selectively permeable. The relative distribution of these ions is similar in most excitable cells. Potassium is concentrated inside of the cell while the opposite is the case for the others. Typical ionic concentrations for mammalian neurons are listed in Table 3.1. The difference in a specific ion concentration on each side of the membrane causes the permeant ion to move down its chemical gradient. Since ions are charged entities, this movement is accompanied by a charge build-up at the membrane, because the counterions are not able to pass through the selectively permeable membrane. This leads to the formation of a potential difference acting against the diffusion 
gradient. At some point, the opposing forces reach equilibrium and an electrochemical gradient is formed. The potential, at which the system is at equilibrium, is given by the Nernst equation.

$$
E_{e q}=\frac{R T}{z F} \ln \frac{[\text { ion }]_{o}}{[\text { ion }]_{i}}
$$

$E_{\text {eq }}:$ equilibrium potential of the specific ion

$R$ : gas constant $8.315 \mathrm{~K} \cdot \mathrm{mol}^{-1}$

$T:$ absolute temperature

$z$ : valence of the ion

$F$ : Faraday's constant $93485 \mathrm{C} \cdot \mathrm{mol}^{-1}$

[ion]: the specific ion concentration outside and inside of the cell

The equilibrium potentials calculated for each of the above-mentioned ions are listed in Table 3.1. In a system with a single permeant ion, the membrane potential would be equal to the equilibrium potential of that ion. However, excitable cells at rest are permeable to $\mathrm{K}^{+}, \mathrm{Na}^{+}$, and $\mathrm{Cl}^{-}$. Thus, the membrane potential lies between the individual ion equilibrium potentials, and the contribution of each ion is weighted by its permeability as demonstrated by the Goldman-Hodgkin-Katz equation.

$$
V_{m}=\frac{R T}{F} \ln \frac{p_{K}\left[K^{+}\right]_{o}+p_{N a}\left[N a^{+}\right]_{o}+p_{C l}\left[C l^{-}\right]_{i}}{p_{K}\left[K^{+}\right]_{i}+p_{N a}\left[N a^{+}\right]_{i}+p_{C l}\left[C l^{-}\right]_{o}}
$$

$V_{m}:$ membrane potential

$p$ : relative permeability

Since the resting membrane is mostly permeable to potassium ions, the membrane potential of most cells is negative, in neurons typically between -75 and $-60 \mathrm{mV}$.

Table 3.1: Ion concentrations and calculated equilibrium potentials for mammalian neuronal cells. Table adapted from [15].

\begin{tabular}{llll}
\hline Ion & $c_{\mathrm{i}}(\mathrm{mM})$ & $\mathrm{c}_{\mathrm{o}}(\mathrm{mM})$ & $\mathrm{E}_{\text {eq }}(\mathrm{mV})$ \\
\hline $\mathrm{K}^{+}$ & 140 & 4 & -95 \\
$\mathrm{Na}^{+}$ & 18 & 145 & +56 \\
$\mathrm{Ca}^{2+}$ & 0.0001 & 1.2 & +125 \\
$\mathrm{Cl}^{-}$ & 7 & 120 & -76 \\
\hline
\end{tabular}




\subsection{Action potential}

The action potential is a propagating electrical activity caused by a fast, temporary, and localized shift in the membrane potential. In 1939, Cole and Curtis showed that the action potential is accompanied by a large decrease in the membrane impedance, giving evidence for the membrane permeability change during the activity. ${ }^{16}$ Building upon the knowledge of the decades before, Hodgkin, Huxley, and Katz managed to give a qualitative and quantitative description of the mechanism of the action potential formation and propagation using the giant axon of Loligo. ${ }^{17-21}$ The action potential is an all-or-nothing event. ${ }^{17}$ It is triggered by a membrane potential depolarization reaching the threshold for action potential firing, typically $10-15 \mathrm{mV}$. The initial trigger causes further depolarization with the membrane potential shifting to positive values, followed by repolarization and slight hyperpolarization. By application of ion substitution and the voltage clamp technique, Hodgkin and Huxley were able to identify that the rapid, depolarizing, inward current was caused by the increased conductance of $\mathrm{Na}^{+}$, while the delayed, slower, repolarization, outward current was carried by $\mathrm{K}^{+}$(Figure 3.1). ${ }^{21}$ The unidirectional spread of the action potential is ensured by a rapid $\mathrm{Na}^{+}$ current inactivation. The concentration gradients of the cell are not altered during the action potential, however the amount of charge transferred across the membrane capacitor is sufficient to cause the large deviations from the resting membrane potential. The ionic misbalance is brought back to the original equilibrium by the active transport of the $\mathrm{Na}^{+} / \mathrm{K}^{+}$ pump.

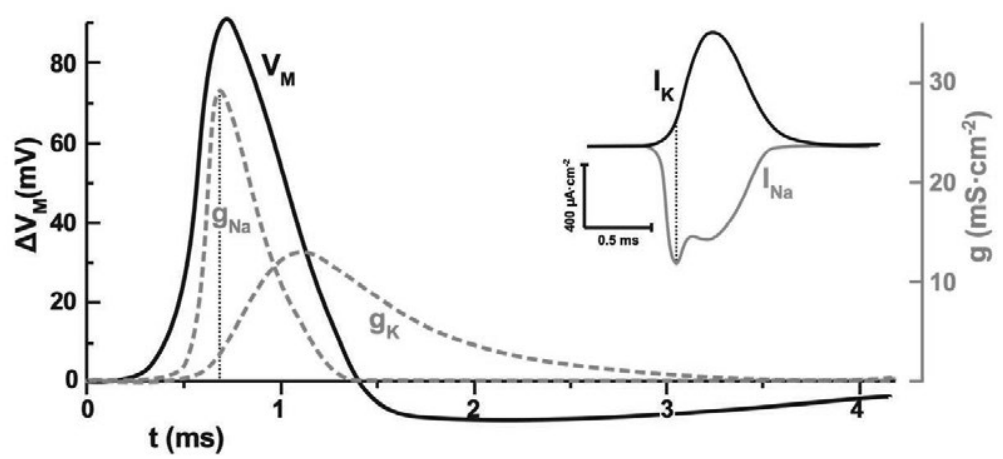

Figure 3.1: A time-course of the action potential and the related changes in the sodium and potassium conductance (current in the inset) for a giant axon of Loligo calculated by the Hodgkin \& Huxley numerical model. Figure adapted from [21]. 


\subsection{Ion channels}

The speed at which ions flow through the membrane during an action potential is too high to be achieved by an active transport system or by carrier proteins. The change in permeability is caused by opening of transmembrane pores called ion channels which allow passive diffusion of selected ions down their electrochemical gradient. The opening of the ion channels is caused by several kinds of stimuli, which is sometimes used for their classification. Ion channels can respond to a change in the membrane potential (voltage-gated), neurotransmitter and chemical binding (ligand-gated), or a sensory input as in light-gated channels, mechanosensitive channels etc. Some ion channels can respond to multiple stimuli, for example the transient receptor potential vanilloid 1 channel (TRPV1) is activated by heat, capsaicin binding, membrane potential shift, extracellular $\mathrm{pH}$ change, and others. ${ }^{22}$ The spread of the electrical signal in excitable cells discussed above is caused by the action of voltage-gated sodium and potassium channels. However, the initial trigger, the $10-15 \mathrm{mV}$ depolarization, is caused by opening of ligandgated, mechanosensitive or other ion channels, generally called receptors. The triggering of an action potential in neural cells can be caused by a direct sensory input or by information transduction from another neuron at a synapse. In some cases, the receptors are located directly in the membrane of a sensory neuron, while in other cases specialized receptor cells are present that pass on the signal to the sensory neuron via synapses. It is evident, that artificial communication with excitable cells can be done at two stages. One can either activate the receptor channels by a local delivery of neurotransmitters and other activating molecules; or one can act on the voltage-gated ion channels by depolarizing the membrane as is done in electrical stimulation.

\subsubsection{Voltage-gated ion channels}

In the animal kingdom, there are three major classes of voltage-gated ion channels classified by the ion they are permeable to, the Nav, $\mathrm{K}_{\mathrm{V}}$ and $\mathrm{CaV}$ channels. ${ }^{15}$ The channel pore is formed by tetrameric structures of four identical subunits $\left(\mathrm{K}_{\mathrm{V}}\right)$ or four homologous domains of a single subunit $(\mathrm{Na} v, \mathrm{Ca})$, each composed of six transmembrane $\alpha$-helix segments. Voltage-gated ion channels have two crucial characteristics, they contain a voltage sensing domain acting as the gate opening mechanism and a selectivity filter, which allows the passage of a specific ion. ${ }^{15}$ The voltage 
sensor is composed of the S4 transmembrane $\alpha$-helices containing positive amino acid residues called gating charges. During membrane depolarization, the four individual S4 segments move outward causing conformational changes to open the channel. The ion specificity is given by the amino acid composition of the narrowest part of the channel pore. The selectivity filter provides specific interactions only with the permeant ion. The voltage-conductance characteristics of the channels can be conveniently studied by the voltage clamp technique (Section 6.3.5). Because excitable cells express a number of voltage gated channels, the study of their individual contributions is complicated, but possible by implementation of neurotoxins, other channel blockers (drugs and ions), or ion substitution. Alternatively, the ion channels can be expressed in a heterologous system with a low level of endogenous channels. The most popular systems are the human embryonic kidney cells and Xenopus laevis oocytes. Potassium channels are the most diverse family of ion channels exhibiting a number of different functions. The Shaker $\mathrm{K}_{\mathrm{V}}$ channel from Drosophila melanogaster, was identified due to its mutation causing flies to shake their legs upon exposure to ether. ${ }^{23}$ Shaker was the first $\mathrm{K}_{\mathrm{V}}$ channel cloned and is one of the best understood ion channels to date. ${ }^{24}$ Because of the deep understanding and a number of useful mutations available, ${ }^{25,26}$ it is a great model for voltage-gated ion channels.

\subsection{The in vitro model - Xenopus laevis oocyte}

Xenopus laevis oocyte is a popular expression system for the study of ion channels due to its high translation efficiency and low endogenous ion channel levels. Messenger RNA transcribed from the coding DNA corresponding to the ion channel of interest is injected in the oocyte for translation. The advantage of the system is its ability to translate a wide range of eukaryotic mRNA. ${ }^{27}$ Because of the large cell size, approximately $1 \mathrm{~mm}$ in diameter, the oocytes are very easy to handle both during the mRNA injection and the subsequent electrophysiological recording by two-electrode voltage clamp. Since each cell has to be handled individually, the process of mRNA injection can be time consuming, but allows for high yield of expressing cells and easy protein co-expression in comparison to mammalian cell transfections. Another major advantage of the oocyte system is the absence of need for cell culture on top of the studied electronic device. The oocytes are not 
attaching cells, after the injections they are kept in solution and are individually placed in the target position within the measurement setup just before the experiment. Furthermore, the cells are very robust thus allowing for long continuous measurements $(\sim 1 \mathrm{~h})$.

\subsection{Peripheral nervous system}

The nervous system is a complex machinery in charge of collection and transmission of information throughout the body. The two main components are the central nervous system (CNS) and the peripheral nervous system (PNS). The CNS comprises the brain and the spinal cord, while the PNS is composed of nerves and ganglia connecting the rest of the body with the $\mathrm{CNS}^{28} \mathrm{~A}$ nerve consists of nerve fibers (axons) carrying sensory (afferent), control (efferent), and autonomic signals to and from the CNS. The autonomic nervous system sends signals to cardiac muscle, smooth muscle, and glands. The autonomic system is subdivided to sympathetic "fight-or-flight" and parasympathetic "restand-digest" systems.

Some nerve fibers are myelinated, their segments are covered by myelin sheaths made of enwrapped Schwann cells and separated by nodes of Ranvier, which are the only access points to the axon membrane. The nodes are also the only spaces with ion channels present. Myelination serves a similar purpose as cable insulation; it shields the axon from other electrical signals in the vicinity. It also reduces the membrane capacitance while increasing the membrane resistance and thus speeds up the signal conduction velocity. Each nerve fiber is enclosed by a connective tissue called endoneurium. Fascicles are collections of tightly packed fibers enclosed by high resistance perineurium connective tissue, which acts as the blood-nerve-barrier. ${ }^{29}$ The fascicles collect nerve fibers based on their destination, not the functionality, thus afferent and efferent fibers of the same target are grouped together. The nerve, consisting of one or more fascicles, is enclosed by epineurium giving the nerve a tensile strength.

Skeletal muscle fibers are controlled by somatic motor neurons, the somas of which are located in the spinal cord, while the axons are protruding out through the ventral routes. ${ }^{30}$ One motor neuron typically innervates a number of different muscle fibers; the system of the motor neuron and its muscle fibers is called a motor unit. The neuromuscular junction is a specialized synapse between a motor neuron and a muscle 
cell. It is mediated by acetylcholine released from the neuron synaptic vesicles, which binds the acetylcholine-gated channels in the motor end plate of the muscle fiber. The channel is permeable to both $\mathrm{Na}^{+}$and $\mathrm{K}^{+}$, but $\mathrm{Na}^{+}$is the predominant ion. The sensory neurons bring information from different kinds of receptors (as discussed above) through the dorsal roots of the spinal cord into the CNS. The electrical activity of nervous or muscle systems is registered as compound action potentials (CAPs), which are the result of simultaneous activity of a number of fibers or cells. The amplitude of CAPs depends on how many units have been recruited.

\subsection{The in vivo model - rat sciatic nerve}

The sciatic nerve is the largest nerve in the body. It is responsible for stimulation of the leg muscles and for carrying the sensory signals from the leg to the CNS. The rat sciatic nerve is one of the most common models for peripheral nerve studies, such as nerve injury, regeneration and recovery. ${ }^{31,32}$ In the field of bioelectronics, it is a frequently-studied model for peripheral nerve interfaces. The nerve size is moderate (1$1.5 \mathrm{~mm} \varnothing)$, it offers a straightforward validation and rats can be easily trained for behavioral tests. The rat sciatic nerve is formed mainly by fibers from the L4-L5 spinal segments with a small, variable contribution from the L6 and L3 segments. ${ }^{33}$ It separates first into two and then into four fascicles, i.e. tibial, sural, peroneal, and cutaneous. The cutaneous nerve is the smallest branch, which separates in the mid-thigh. The tibial, sural and peroneal branches split in a trifurcation above the knee. The sciatic nerve comprises approximately 27000 axons of which $6 \%$ are myelinated motor axons, 23\% myelinated afferent axons, 48\% unmyelinated afferent axons, and 23\% unmyelinated sympathetic axons. ${ }^{34}$ Most of the motor fibers are located in the tibial (1000) and peroneal (600) nerves. The sural branch apparently contains about 80 motor axons, while the cutaneous nerve seems to be exclusively afferent. ${ }^{34}$ The tibial nerve is the largest branch and is in charge of innervating the posterior of the leg.

Nerve damage can be exhibited as a loss of motor function, impaired sensation, or neuropathy. A number of techniques can be used for evaluation of the nerve health. ${ }^{35}$ Functional tests examine the ability to perform motor and sensory actions. Walking track analysis is a typical method for quantification of motor recovery in peripheral nerves. It is based on footprint analysis of the hind legs. ${ }^{36}$ The sensory function and 
pain response can be tested by the application of von Frey hair test, which involves pressing filaments of increasing stiffness against the foot and noting the animal's reaction. In essence, functional tests are qualitative rather than quantitative, since most responses are quite complex. A more quantitative approach to examining the nerve health and functionality are electrophysiological methods. Typical parameters assessed are the conduction velocity, the compound action potential amplitude and area, and the latency after an artificial stimulation, if applicable. The most accurate (and most invasive) method for assessment of nerve health is histology, i.e. the study of the microanatomy of the specimen. The tissue is typically sectioned, stained and observed under light or electron microscope. Immunoresponse and very fine damage such as demyelination, axonal loss, and axonopathy can be detected. However, the technique is very laborious, time consuming and can only be performed on fixed tissue (after the experiment). 


\section{Excitable tissue stimulation}

Artificial stimulation of excitable cells is possible by depolarization of the cell membrane, which can be triggered either chemically or electrically. Chemical stimulation can be based on local delivery of signaling molecules or on rapid change in the ionic composition at the cell membrane. Electrical stimulation depolarizes the cell by application of depolarizing current from an electrode. Chemical stimulation is by virtue more selective than electrical due to the substance specificity to the target. However, chemical delivery device response time is not fast enough for some applications, as a few ms delay time remains the hard limit for most technologies. ${ }^{37,38}$ Furthermore, chemical delivery systems require drug reservoirs, which have to be periodically refilled, thus presenting a hurdle for chronic applications. On the contrary, electrical stimulation is generally easier to implement, multiplex and chronically maintain.

\subsection{Chemical stimulation}

There are many techniques for application of drugs into the body. The most common administration is systemic in the form of oral tablets or injections into the blood stream. More specific and long-lasting therapy can be based on implanted drug releasing systems. Such delivery is typically based on slowly dissolving polymeric structures with entrapped drugs. The release is passive and happens at a fixed rate pre-determined by the biodegradability of the structure. Passive systems do not provide flexibility in the delivery dynamics and thus there is significant research effort invested into developing active drug delivery systems.

Precision medicine is a new medical paradigm aiming for tailored patient care accounting for individual differences in the treatment response. A part of the effort is having controlled drug delivery devices allowing for local, on-demand, and accurate dosing of substances to increase the patient outcome and reduce the side effects. Electrical drug delivery systems based on integrated circuits provide excellent control over the substance release and enable individually operated, multidrug, multisite release points. The integrated circuit usually contains a powering unit, a control unit and a drug release unit. For closed-loop self-regulating systems, sensing and data communication components would also be 
present. The most used electronic drug dosing therapy in the world is the insulin micropump for diabetic patients to assure more controlled medication. ${ }^{39}$ There are a large number of mechanisms to provide electrical control over drug release, a few of which are discussed below.

\subsubsection{Micropumps}

Electronic micropumps are devices causing spatial displacement of the drug from a reservoir to the target medium. Therefore micropumps can be used to deliver virtually any soluble agent. The pumping mechanism is based on fluid displacement by a pressure-controlled diaphragm. Most micropump drivers work on piezoelectric, thermopneumatic, electrostatic, or electrochemical effects. ${ }^{40}$ Piezoelectric materials change their volume depending on the applied potential, thus deflecting the diaphragm. Electrostatic drivers contain two parallel plate electrodes, which move depending on the applied potential between them. By connecting one of the electrodes to the diaphragm, the latter can be actuated. The thermopneumatic and electrochemical drivers contain a liquid-filled chamber above the diaphragm. The chamber expands as the working liquid in the thermopneumatic device is resistively heated or when the liquid in the electrochemical driver is electrolyzed, producing gaseous products. The liquid displacement causes pressure build-up at the outlet of the micropump, which can be limiting for certain applications.

\subsubsection{Conducting polymer electrostatic actuators}

Conducting polymers can be used as minimalistic electronically controlled drug release devices due to their redox chemistry. By application of electric potential, conducting polymers can be switched from a neutral to a charged state. Upon charging, counterions have to be incorporated into the polymer to maintain charge neutrality. If the counterion is a drug, upon electrical switching back to the neutral state, the drug is released. Most biologically-stable conducting polymers are p-type and thus switch between neutral and oxidized state. ${ }^{41}$ This configuration allows for uptake and release of negatively charged drugs. Modifications of the mechanism by incorporating immobilized counterions or neutral molecules linked to a charged moiety makes release of cationic and neutral drugs possible. ${ }^{42}$ The main disadvantage of conducting polymer based electrochemical drug delivery systems is their low drug loading and passive diffusion. 


\subsubsection{Electrophoretic delivery}

Electrophoresis describes the movement of charged substances in an electric field. An example of such a drug delivery system is the organic electronic ion pump (OEIP). OEIPs are composed of a source reservoir and an ion exchange membrane, which separates the source from the target (Figure 4.1). By applying an electric field between the drug reservoir and the target, cationic or anionic substances/drugs selectively migrate through the ion exchange membrane to the target electrolyte, cell culture or tissue of interest. ${ }^{8,43}$ The membrane is typically a polyelectrolyte, i.e. a polymer with fixed specific charge. The polarity of the membrane determines which ions will pass through. Polycations, or anion exchange membranes, will selectively pass anions. Conversely, polyanions, or cation exchange membranes, transport only cations. Selectivity can be further assured by loading aqueous solution of the drug to the source electrolyte without any buffer component. In this way, the drug is the only available ion for transport. The transport of a drug is limited by the membrane conductivity, the interactions between the membrane and the pumped ion and the drug's reactivity. The main advantage of the OEIP technology is the so-called "dry delivery", meaning that the device transports almost exclusively the ions, but not the solvent. This characteristic is important especially in applications where exerting pressure on the target tissue is unwanted. ${ }^{44,45}$

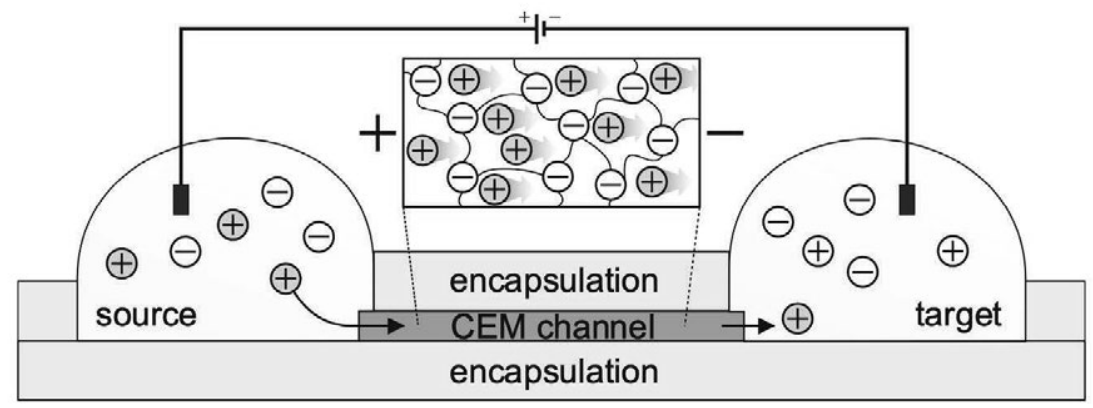

Figure 4.1: Schematic of an organic electronic ion pump. The mechanism is demonstrated on a device for transporting ("pumping") positively charged entities. The channel is made of a polyanion acting as a cation exchange membrane (CEM). The electrodes in contact with the source and target electrolyte must provide sufficient capacity to assure continuous operation. 


\subsection{Electrical stimulation}

A number of medical conditions are clinically treated by application of stimulation electrodes. The most common applications are cardiac pacemakers, cochlear implants for treating deafness, spinal cord stimulators to reduce chronic pain, deep brain stimulators for motor disorders such as Parkinson's disease, artificial retinas for vision, and peripheral nerve stimulation for somatic and autonomous nervous system disorders. In neuromodulation therapy, we are interested in stimulating excitable tissue with extracellular electrodes in a non-disruptive way. This is in contrast to the electrophysiological techniques used for the study of transmembrane currents involving invasive intracellular or patch electrodes applied in basic science. This subchapter concerns the extracellular electrical stimulation mechanism, electrode characteristics, and alternative, wireless approaches to elicit cellular stimulation.

\subsubsection{Electrical stimulation mechanism}

When a cell is placed on a cathodically polarized planar electrode, it becomes depolarized on the side interfacing the electrode, resulting in the opening of voltage-gated sodium channels and artificial triggering of an action potential. The local depolarization is caused by the change of the local electric potential outside of the cell due to the flowing electric currents. This will lead to local charging of the cell membrane, which the intracellular cations will screen by redistributing from the distant parts of the cell, causing slight hyperpolarization in the other areas (Figure 4.2). The stimulation mechanism was elucidated in detail by Fromherz and coworkers using capacitive electrodes on single semi-spherical excitable cells, ${ }^{46}$ but is also applicable to different geometries such as neural fibers. ${ }^{47}$ Anodic stimulation can also trigger action potentials, since it leads to depolarization of the distant cell region. However, because the effect on the distant region is less pronounced, anodic configuration necessitates significantly higher stimulation currents. ${ }^{47}$ 

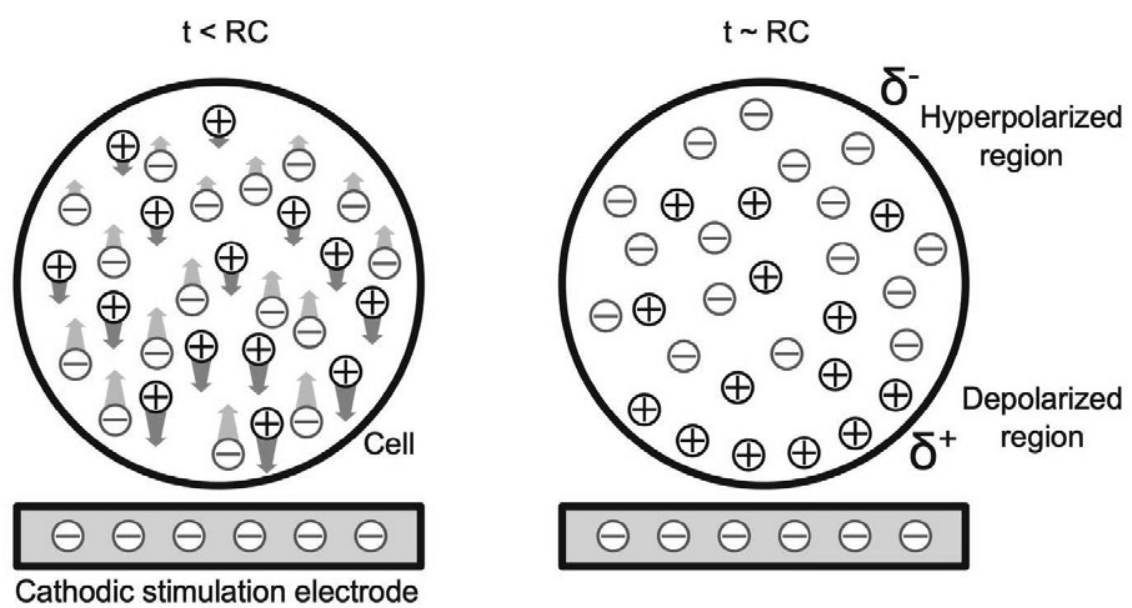

Figure 4.2: The mechanism of capacitive cell stimulation. At the onset of the stimulation pulse, the intracellular cations are attracted towards the cathode thereby depolarizing the region. During the electrode charging (RC), transient voltage is generated thus affecting the cell membrane polarity.

\subsubsection{Electrode characteristics}

Charge is carried differently in the electrode and the surrounding tissue. In electronic circuits, the main carriers are electrons, while in biological tissue, charge is carried by ions. Therefore, transduction between electronic and ionic current is necessary. Two different charge transfer mechanisms occur at the electrode-electrolyte interface. ${ }^{47}$ The first is purely capacitive and relies on the reversible reorganization of the ions in the electrolyte and the formation of a charged double-layer at the electrode interface (Figure 4.3). This mechanism is called capacitive or non-faradaic and is considered safer for chronic stimulation. The doublelayer capacitance is typically $10-20 \mu \mathrm{F} / \mathrm{cm}^{2}$ for a polished metal electrode. The second mechanism can arise if the potential of the electrode reaches the electrochemical potential of one or more species present in the electrolyte, leading to redox reactions. This mechanism is termed faradaic and includes electron transfer between the electrode and an oxidized/ reduced species in the electrolyte. Faradaic reactions are generally considered unsafe in the neurostimulation paradigm due to possible degradation of the surrounding tissue by the reaction products $(\mathrm{pH}$ alteration, reactive oxygen species production, gas formation, nutrient depletion etc.) or corrosion of the stimulating electrode. ${ }^{47}$ 
However, if the redox reactions are reversible and the electrochemical products remain at the electrode surface, the reverse reaction can take place during the second phase of charge-balanced biphasic pulse stimulation, and no net (toxic) products should be formed. As long as reversible operation is granted, faradaic electrodes are commonly used in neuromodulation because their charge injection capacities are larger than those of capacitive electrodes. It is important to note that neural damage can occur both with faradaic and capacitive stimulation when the injected charge, or the charge density is too high. ${ }^{48,49}$
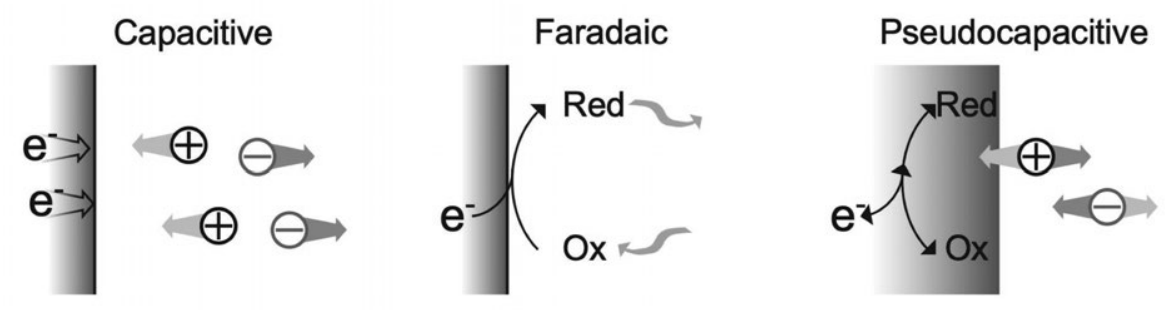

Figure 4.3: Charge transfer mechanisms between the electrode and an electrolyte solution.

For stimulating electrodes, capacitance should be as high as possible in order to be able to inject sufficient charge during stimulation. The figure of merit for recording electrodes is impedance, which should be minimized for the signal-to-noise ratio to be high. The impedance of stimulation electrodes should also be kept minimal in order to reduce the driving voltage necessary for passing the stimulating current. Typical stimulation electrodes are made of noble metals such as platinum, iridium, platinum iridium, and to some extent palladium, and gold, offering charge injection capacity of up to $300 \mu \mathrm{C} / \mathrm{cm}^{2} .{ }^{50}$ These figures are sufficient for most macroscopic electrodes. However, materials with higher charge injection capacities are necessary for microelectrode applications. Both capacitance and impedance can be optimized by increasing the area of the electrode. This can be achieved either by making the electrode larger (macroelectrode), or by structuring to increase the effective surface area. Sputtering and electrochemical deposition are methods intrinsically suitable for facile production of nanostructured surfaces. These techniques afford titanium nitride (TiN), $\mathrm{Au}$, or $\mathrm{Pt}$ microelectrodes with charge injection capacities of 1-3 $\mathrm{mC} / \mathrm{cm}^{2} .{ }^{50-53}$ Another approach to increasing the effective surface area 
includes coating of the metal electrodes with a pseudocapacitive threedimensional material, i.e. a material exhibiting reversible redox reactions that change solely the valence of the coating. ${ }^{50}$ The most commonly used 3 -dimensional pseudocapacitive material is iridium oxide involving $\mathrm{Ir}^{3+} / \mathrm{Ir}^{4+}$ pairs and providing charge injection capacities up to $5 \mathrm{mC} / \mathrm{cm}^{2} .{ }^{50}$ Conducting polymers are a promising, cheaper alternative for pseudocapacitive coatings. The full volume is accessible to the reaction and thus the electrode capacitance increases with the coating thickness. This characteristic has been referred to as the volumetric capacitance. ${ }^{54}$ Poly(3,4-ethylenedioxythiophene) blended with the polyanionic "dopant" polystyrene sulphonate (PEDOT:PSS) has become by far the most widely explored conducting polymer coating, predominantly in recording electrodes. ${ }^{55}$ PEDOT:PSS electrodes offer high charge injection capacities of $15 \mathrm{mC} / \mathrm{cm}^{2}$ allowing for extremely small stimulation microelectrodes. However, conducting polymer coatings typically suffer from delamination during operation. ${ }^{50}$ Deposition on structured surfaces improves the adhesion. ${ }^{56,57}$ Nevertheless, long-term chronic stability of the PEDOT:PSS interface has yet to be proven and safe levels of operation have to be further studied due to the relative ease of cathodic hydrogen peroxide production on PEDOT:PSS electrodes. ${ }^{49,58}$

\subsubsection{Direct wireless stimulation}

Electrical stimulation is an important technique both for basic research and for translational medicine. However, the necessity of wiring and the limit on complexity makes certain types of experiments and medical applications impossible. The ability to modulate cells in a remote way is highly enabling both for in vitro and in vivo applications and thus the interest in wireless stimulation techniques is ever growing. ${ }^{59}$ Wireless power transfer systems can be used to operate conventional electrical stimulators, a topic of a subsequent chapter. However, certain types of energy can be used to modulate cellular behavior directly or in combination with a simple transducer. The most pursued technologies are based on ultrasonic waves, switching magnetic fields, or optical power.

Ultrasound in the range of 0.1 to $3 \mathrm{MHz}$ has high penetration depth and can be focused on target tissue to modulate the nervous system without the use of exogenous transducers. Studies demonstrating selective activation of different organs have been shown. However, the mechanism 
of neural activation is not fully understood to-date. ${ }^{60,61}$ The proposed effects are thermal, mechanical due to the acoustic force, or cavitational, and depend on the ultrasound frequency and intensity. ${ }^{62}$ Specificity of focused ultrasound can be further improved by utilizing piezoelectric particle transducers that generate current upon ultrasound stimulation and can thus modulate voltage-gated ion channels. ${ }^{63}$ In a similar manner, magnetic particles in combination with alternating magnetic field can be used to stimulate excitable cells. Depending on the particle size and the magnetic field switching frequency, the particles can either modulate mechano-sensitive ion channels ( $\mu \mathrm{m}$ scale, slow switching) or thermallyactivated ion channels (nm scale, radio frequency range). ${ }^{64-66}$

\subsubsection{Light stimulation}

Light can be used as stimulus for cellular excitability in various ways. The most selective approach is optogenetics, which is based on genetic modification of the target cells making them inherently photosensitive. In other approaches, light in combination with a transducer is used to change the membrane properties indirectly through changes in the physical and/or chemical environment of the membrane. The transducer can be either naturally present (e.g. water in combination with infrared light) or can be introduced into the system to achieve better spatial or functional selectivity (light-absorbing molecules, nanoparticles, microparticles, or devices). The different mechanisms through which the stimulation can happen are photothermal, photochemical, photofaradaic, and photocapacitive, or some combination. ${ }^{59,67}$

Many systems working on various wavelengths of light have been reported, both for in vitro and in vivo applications. ${ }^{59,68-72}$ The latter is possible either by implantation of optical fibers and light emitting diodes or by transmitting light within the so-called tissue transparency window. There are two regions on the visible-IR spectrum, where the body pigments and water molecules show minimal absorption. The first tissue transparency window is in the range of $650-950 \mathrm{~nm}$, while the other is $1000-1350 \mathrm{~nm}$ (Figure 4.4). ${ }^{73}$ Thus, transducers operating within these wavelengths can be used relatively deep inside of the tissue. Light penetration changes with its wavelength, since each tissue has wavelength dependent light absorption and scattering constants. ${ }^{74}$ Each tissue also has a specific anisotropy factor, which has to be taken into account especially for light delivery through multilayer structures. ${ }^{75}$ 
Light delivery into the tissue is often discussed in terms of penetration depth, which denotes where the beam intensity drops to $1 / \mathrm{e}$ (approximately 37\%) of its incident value. ${ }^{75}$ However, it does not mean that some photons do not travel further. Therefore, speaking only in terms of penetration depth can be misleading. The necessary incident beam intensity value should be calculated based on the sufficient intensity at the depth of the implant while keeping the light safety limits in mind. The maximum permissible exposure limits for skin and ocular irradiation are given by the light source wavelength, the illumination pulse duration and the duty cycle; specific equations are listed by national standards. ${ }^{76}$ Light driven stimulators are especially suited for applications in sight restoration due to the easy light in-coupling into the eye. However, even in vision applications, using wavelengths outside of the visual spectrum is preferable, especially in patients with residual peripheral vision in order to avoid phototoxic and photophobic reactions. ${ }^{72}$

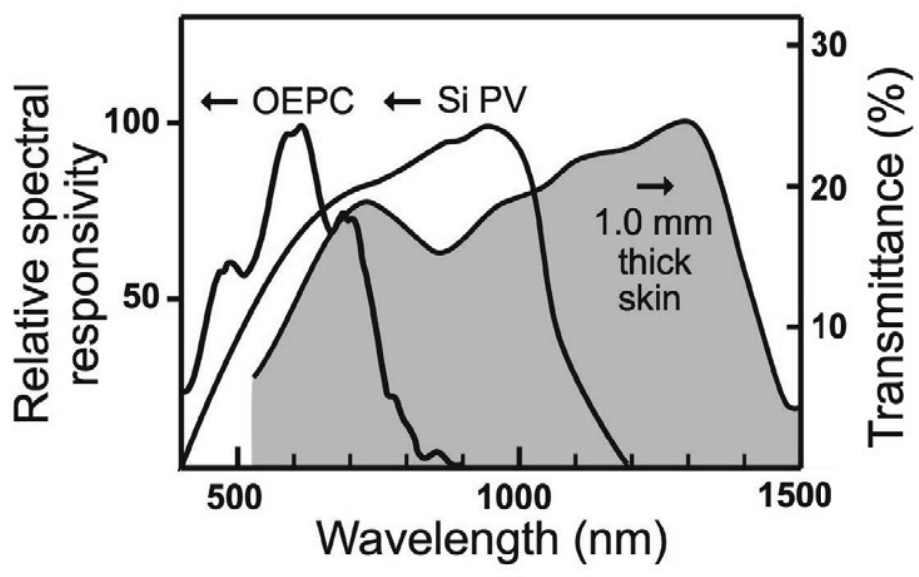

Figure 4.4: Overlap of human skin transmittance spectrum with spectral responsivities of typical silicon photovoltaic and the organic electrolytic photocapacitor device studied in this thesis. Original data adapted from $[7,77]$.

\subsubsection{Optogenetics}

Optogenetics relies on genetic introduction of a light-gated ion channel in the membrane of the target excitable system. The modified cells can then be activated by light delivery. Optogenetics is an elegant method, which is extremely specific, and can be multiplexed by the use of several channels sensitive to different wavelengths. However, the necessary 
genetic manipulation of the target organism keeps the application in humans elusive. Furthermore, the availability of ion channels responding within the tissue transparency window is limited. ${ }^{78}$ Thus the necessity to deliver sufficient light to the target location requires implantation of optical fibers and other complex circuits, making optogenetics roughly as invasive as conventional electrical stimulators. ${ }^{79}$

\subsubsection{Photothermal mechanism}

Direct photothermal stimulation is possible with infrared light of $1.5 \mu \mathrm{m}$ wavelength and higher, which is absorbed by water molecules in the tissue, and causes localized heating leading to action potential triggering. Two mechanisms have been identified to cause this effect depending on the light power. If the light intensity is high, fast temperature change in the membrane vicinity increases its electrical capacitance, which causes depolarizing currents. ${ }^{80}$ With lower light intensities, the rate of temperature change is not high enough to cause changes of the membrane capacitance and a different mechanism is in place. Longer pulses are necessary to depolarize the membrane. The temperature change leads to the opening of temperature sensitive ion channels (TRPV family). ${ }^{81}$ The two mechanisms of photothermal stimulation are valid also when using transducers such as plasmonic nanoparticles, ${ }^{82}$ polymer films, ${ }^{83}$ and semiconductor micro ${ }^{84}$ and nanoparticles. ${ }^{85}$

\subsubsection{Photochemical mechanism}

Photochemical stimulation refers to reactions occuring on a single material or chemical moiety. There are two groups of photochemical reactions that can modulate cellular electrophysiology. The material can either undergo a photoredox reaction or a photoisomerization reaction. In the case of photoredox chemistry, photogenerated holes and electrons react with reducers and oxidants available in the surrounding medium. The principle of charge neutrality has to be followed and thus both sides of the redox reaction must be balanced. This concept is well characterized for organic semiconductors, where the prevalent reaction is oxygen reduction accompanied by autooxidation or other available substrate oxidation. ${ }^{86}$ Reactive oxygen species have been shown to modulate certain ion channels but further exploration into the general mechanism of action is needed. ${ }^{87,88}$ In the other case of photoisomerization chemistry, also called photoswitching, the material undergoes reversible isomerization when exposed to a certain wavelength of light. The reaction alters the structure, and thus the properties, of the molecule. This 
molecular reorganization changes the way the molecule interacts with the target ion channel and thereby modulates the channel's conductance. ${ }^{89}$

\subsubsection{Photofaradaic mechanism}

Photofaradaic stimulation applies to semiconductor devices with a builtin electric field in the form of a heterojunction or Schottky barrier. ${ }^{90}$ Upon illumination, a photon gets absorbed by the semiconductor and forms an exciton. Due to the electrochemical potential asymmetry within the device, the exciton can be separated into a free hole and a free electron, which can react with the surrounding medium. Photofaradaic devices stimulate by the faradaic current that flows from the positive to the negative terminal of the device, but the reaction products can also play a role. ${ }^{67}$ Similarly to photochemical redox reactions, both electrons and holes have to react in order to close the circuit. Therefore, if reactions are not available at both sides of the device, photofaradaic current will not occur. $^{91}$

\subsubsection{Photocapacitive mechanism}

Photocapacitive stimulation refers to the same device architecture as photofaradaic, however, the photogenerated charge accumulates at the material-electrolyte interfaces thus producing two charged double-layers of opposite polarity. The device architecture must account for spatial charge separation in order to shape the electric potential formed in the electrolyte. ${ }^{90}$ In the photocapacitive stimulation paradigm, it is the capacitive currents that cause membrane depolarization and thus opening of the voltage-gated ion channels. ${ }^{46}$ An organic semiconductor based device working in this mode, the organic electrolytic photocapacitor, is a central part of this thesis and is described in detail in the next section.

\subsubsection{Organic electrolytic photocapacitors}

The organic electrolytic photocapacitor (OEPC) is a minimalistic stimulation device working on the principle of photocapacitive coupling to the cell. The device is composed of a semitransparent conductor acting as a return electrode and a semiconductor heterojunction photopixel (Figure 4.5). ${ }^{7}$ When the device is placed in an electrolyte solution and the heterojunction is illuminated, an exciton is formed in the absorber layer, which is polarized at the heterojunction. If the built-in field is sufficient, the exciton separates into free carries, which are collected at the semiconductor-electrolyte and the conductor-electrolyte interfaces. In the original work by Rand et al., ${ }^{7}$ the architecture was a circular return 
electrode with a concentric smaller $\mathrm{p}$-n heterojunction pixel. The n-type semiconductor was exposed to the electrolyte, therefore the photogenerated electrons accumulated there, while the photogenerated holes concentrated in the exposed part of the return electrode thereby balancing the charge. The formed electrolytic double layers cause capacitive currents in the surrounding medium, which give rise to transient voltage that can alter the membrane potential of cells in the vicinity. The cells or tissue in contact with the photopixel experience cathodic stimulation, while the reverse happens on the return electrode. ${ }^{92}$ By switching the polarity of the heterojunction, the opposite effect will occur.

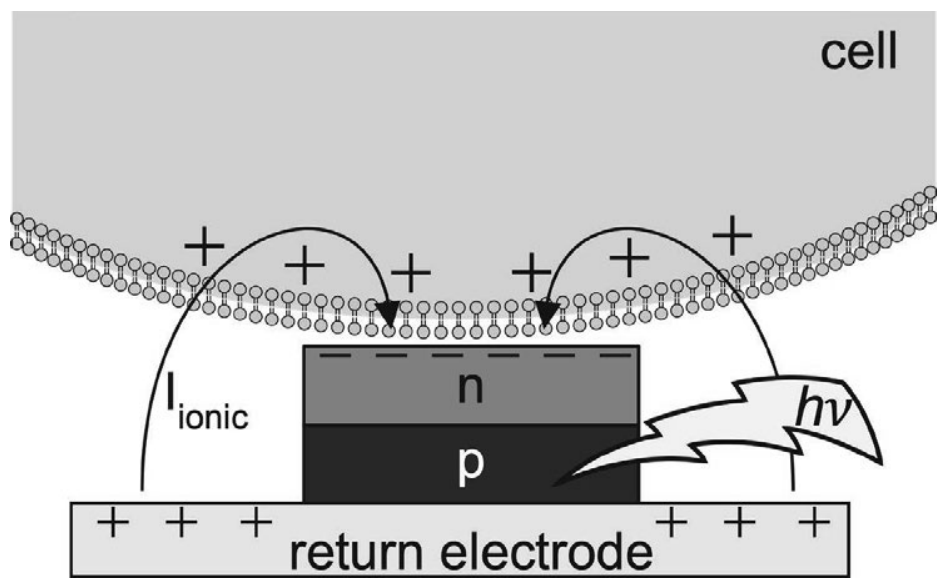

Figure 4.5: Schematic of the organic electrolytic photocapacitor (OEPC) working principle. 


\section{Peripheral nerve interfaces}

The nervous system facilitates many actions in the body ranging from sensation and movement to the correct functioning of glands, cardiovascular and immune systems. Injuries or diseases may disrupt the neural connections leading to a loss of function. The most common approach to restoring or modulating neural function is electrical stimulation and in the field of neuroprosthetics, electrodes are regularly used to stimulate and record neural activity. The most common use of peripheral nerve interfaces is to treat motor conditions. However, there has been an upscaling of non-motor applications in the autonomous nervous system. ${ }^{93,94}$ Most importantly, vagus nerve stimulation has shown efficacy in the treatment of epilepsy, depression, and anxiety and appears to be a promising target for obesity, autoimmune diseases, and a number of others. ${ }^{95}$ In motor applications, one can stimulate the muscles directly, or via motor nerves. The latter is preferred since it requires two orders of magnitude lower stimulation thresholds. ${ }^{95}$

An ideal stimulation/recording interface would possess high selectivity, low stimulation thresholds/high signal-to-noise ratio, and be minimally invasive. Unfortunately, there is a trade-off between these parameters. Typical configurations with an increasing invasiveness and selectivity are extraneural, interfascicular, and intrafascicular electrodes (Figure 5.1). The best selectivity can be achieved with intrafascicular interfaces, since the nerve fibers (axons) can be in direct contact with the electrodes. The stimulation thresholds are typically 3-6 times lower in comparison to extraneural electrodes. ${ }^{96}$ One of the early approaches was to use highdensity penetrating electrode arrays made of silicon (Utah array). ${ }^{97,98}$ Selective stimulation was achieved, but the large, rigid implant showed issues with histopathology. ${ }^{35,99}$ Less-invasive advancements include thin film longitudinal intrafascicular electrodes (LIFE) and transverse intrafascicular multichannel electrodes (TIME), which are implanted either along or across the nerve axis. ${ }^{100}$ The thin film devices offer promising biocompatibility, ${ }^{101}$ however the number of active sites is limited. One of the biggest problems with intrafascicular electrodes is the disruption of the blood-nerve barrier. An intermediate level of specificity and invasiveness is provided by interfascicular electrodes. The perineurium remains intact, thus preserving the blood-nerve barrier, but the stimulation thresholds are higher and the recording signal-to-noise 
ratios lower due to the extra layer of connective tissue. Despite some of the obvious advantages over the intrafascicular electrodes, the interfascicular approach has not gained much attention most probably due to the complicated placement and alignment.

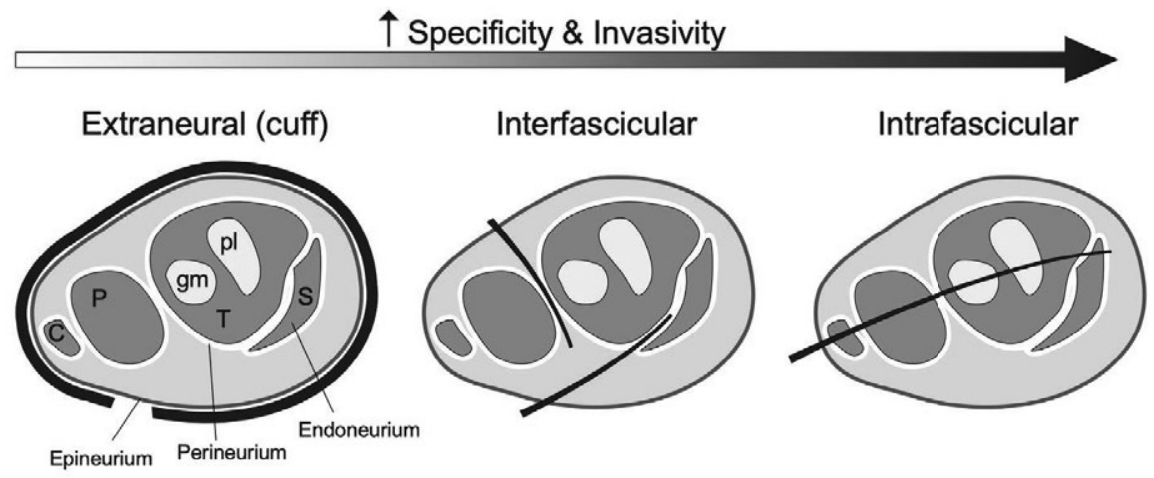

Figure 5.1: Schematic of typical peripheral neural interface electrode placements demonstrated on a rat sciatic nerve anatomy (cross section). The nerve consists of four fascicles: cutaneous $(\mathrm{C})$, peroneal $(\mathrm{P})$, tibial $(\mathrm{T})$, and sural (S). Each fascicle can innervate multiple loci as demonstrated on the tibial branch with the gastrocnemius (gm) and plantar (pl) muscles.

\subsection{Extraneural electrodes}

By far, the most common are the least invasive interfaces i.e. extraneural electrodes placed around the epineurium. Such systems have shown efficacy in patients with spinal cord injury-caused incontinence for up to 16 years ${ }^{102}$ or for footdrop correction up to 12 years. ${ }^{103}$ The most common form is an extraneural cuff with electrodes encapsulated in a polymer sheath ensuring current confinement inside of the nerve. Some specificity in stimulation can be achieved by manufacturing smaller electrodes with high charge injection capacities and by electrode arrangements to allow current steering. ${ }^{104}$ Cuff implantation is relatively easy, as the implants are minimally invasive, leaving the nerve anatomy intact and a number of flexible and stretchable materials are available for their design. However, the extraneural position necessitates higher stimulation thresholds and provides limited selectivity. Selective addressing of subfascicular sections or deeply positioned fibers remains elusive. Cuff electrodes are also not easy to implement in nerves of very small diameter, thereby limiting an approach to increase specificity by 
implanting around nerves at more distal positions. In order to minimize the charge density stimulation thresholds, the electrodes should be as close to the epineurium as possible. However, the cuff inner diameter should be 1.2-1.5 times larger than the nerve outer diameter to reduce compression stress. ${ }^{35}$

Fixing of the cuff electrodes in place is crucial for reproducible operation of the devices. Depending on the implant material and properties, several different strategies have been developed for locking the interface in position. ${ }^{35}$ Most cuff electrodes are built on silicone, which can be fixed by a silicone glue, various three-dimensional locking mechanisms, or by adopting a helical shape. Silicone devices are usually not compatible with microfabrication techniques, thus limiting high-throughput, reproducible production. On the contrary, flexible thin film cuffs can be micromachined and offer smaller mechanical footprint. Thin film interfaces are typically 5-20 $\mu \mathrm{m}$ thick polyimide or parylene devices. Due to the pseudo two-dimensional structure and chemical inertness, different fixing strategies are necessary. The device can be heat-shaped into a spiral of a predetermined diameter. ${ }^{105}$ However, temperature-sensitive devices necessitate a mechanical locking mechanism. Promising solutions are based on zip tie, or paper clip like fixtures made of thin flexible substrates. The paper clip design relies on the mechanical properties of the thin film, which has to be flexible enough to allow positioning of the nerve in the cavity, but rigid enough to clip the nerve in place, thus requiring relatively thick films $(\sim 16 \mu \mathrm{m}) .{ }^{106}$ Furthermore, the paper clip fixture has to be designed specifically for the target nerve diameter. The zip tie mechanism is based on a ribbon substrate with locking "teeth" or indentations designed to be fixed by passing them through a locking loop at the other end of the cuff. ${ }^{107,108}$ The zip tie method can accommodate different nerve diameters by including several sets of spaced "teeth" or indentations.

The safety of the implants is a crucial parameter and the implanted interfaces have to be made of stable and safe materials. Any implant will produce a foreign body immune response, which should be minimized in order to protect the tissue and the device. A layer of fibrotic encapsulation normally grows around the implanted site, as well as between the interface and the nerve. This build-up causes increased electrical resistance and leads to higher stimulation thresholds or lower signal-tonoise ratio in recording electrodes. The foreign body response can be 
minimized by using materials with similar mechanical properties as the interfaced tissue. Novel approaches include the use of ultrathin, flexible, stretchable, or soft materials. ${ }^{109,110}$ The impact of the implant on the nerve health can be studied by a number of electrophysiological and functional tests and histology as described in Section 3.6.

\subsection{Wireless powering of biomedical devices}

Apart from the electrode interface, chronic electrical implants include a power source, processing components, and connecting leads. All the parts increase the implant footprint and make the implantation procedure more invasive. The wireless power supplies are traditionally batteries, which are generally large in size and have a limited lifetime, thus requiring additional/repeated surgical interventions during the patient's life span. ${ }^{94,111}$ In some applications, the energy needs are too high to be supplied by batteries in a sustainable manner, and so percutaneous wires have to be employed. These pose a high risk for infection and cause significant patient discomfort. Therefore, there is a strong interest in development of smaller footprint, less invasive, wireless solutions, both for powering of implantable integrated circuits and for direct stimulation purposes. The choice of the powering system will depend on the energy needs of the electronic device, the target placement, the available space for the implant and other application specifics. In terms of power consumption, drug delivery devices and cardiac defibrillators are on the lower end, while muscle stimulators require the highest power for operation. Wireless powering systems for medical implants are generally divided based on the source of the power, whether it comes from an external source, or from the body itself.

\subsubsection{External power transfer systems}

Energy can be transmitted in the form of radio-frequency (RF) electromagnetic waves, ultrasound, or light. Inductive coupling is the most common strategy because of its technical maturity and high efficiency transfer, while ultrasound and optical systems are gaining more interest since they do not cause electromagnetic interference and have the potential for smaller footprint devices. ${ }^{5}$ The different modalities are described in more details below. 


\subsubsection{RF electromagnetic waves}

Inductive coupling is the most developed, common and efficient way to transfer power. By far the most wireless medical implant devices (such as cochlear implants and neurostimulators) approved for use in humans work on the induction principle. ${ }^{112}$ It is based on electromagnetic induction between two coils. A transmitter coil emits an alternating magnetic field, which induces electromotive force in the receiver coil. To achieve high power transfer efficiencies, the distance between the coils has to be smaller than the dimension of the receiver coil. Typical inductive devices are operated between $100 \mathrm{kHz}$ and $20 \mathrm{MHz}$, with the coil dimensions on the order of millimeters to a couple of centimeters. The power transfer efficiency is optimized by minimizing the coils' separation, increasing the magnetic field strength, operating the transmitter at higher frequency, and by providing proper alignment of the coils, and can reach up to $100 \% .^{5}$ Automatic tuning of power/ frequency is typically applied to minimize the effect of distance and dimensionality on the transfer efficiency. ${ }^{111}$ Applying thin film technology is possible, however thin metal layers lead to poor coil quality factor thus lowering the power transfer efficiency. ${ }^{113}$ In summary, inductively coupled systems have excellent efficiency at very short distances $(<\lambda)$, but are sensitive to misalignment and are relatively bulky (mm-cm scale, $>10 \mu \mathrm{m}$ metal thickness). Therefore, making efficient, small, thin, flexible receiving coils remains elusive. However, on the research scale, the size of the transducer has been decreasing over the years and current optimized systems are able to produce sufficient stimulation to rat sciatic nerve down to submillimeter-sized powering element. ${ }^{114,115}$

A rising alternative to inductively powered systems for applications deeper in the body is radiative coupling using propagating electromagnetic waves to transfer energy. ${ }^{5}$ The frequencies are typically higher than in the inductive coupling applications and can be in the radio or microwave range, allowing for the miniaturization of the receiving antenna. Further advantages are that far field energy transfer mechanisms facilitate coupling deeper inside of tissue and the transmission is less sensitive to the coil alignment. However, the transfer efficiency is much lower than in inductive coupling, because at higher frequencies, the attenuation by tissue is higher. That also increases the possibility of tissue damage. These hurdles have to be solved before this technology can be used as a true alternative for batteries. ${ }^{112}$ 


\subsubsection{Ultrasound}

Propagating ultrasound ( $\geq 20 \mathrm{kHz}$ ) waves are a very promising powering system due to their high penetration depth (tens of $\mathrm{mm}$ ), higher safe power flux, and short wavelength $(\sim 0.75 \mathrm{~mm})$, enabling deeply placed, small scale transducing devices. ${ }^{116}$ Furthermore, ultrasound transducers, typically piezoelectric materials, have a wider acceptance angle, thus reducing the effect of misalignment. State of the art stimulators can operate at considerable depth of $70 \mathrm{~mm}$ while keeping a minimal footprint of $1.7 \mathrm{~mm}^{3}$ and offering controlled stimulation by an integrated circuit along with bidirectional communication. ${ }^{116}$ A disadvantage of this technology is that bone tissue shows high attenuation of ultrasound due to the impedance mismatch with the surroundings, thus not making it directly applicable in certain areas, e.g. brain. ${ }^{5}$ Although ultrasound is considered safe, there are still cases of patient discomfort due to pain and perceptible acoustic noise during use, which have to be addressed before a wide spread of the technology. ${ }^{113}$

\subsubsection{Infrared (IR) light}

Photovoltaic cells can be used to power implants by optical charging. ${ }^{5}$ The external power supply can be either a high intensity light-emitting diode or a laser. Sight restoration in the form of artificial retina is an obvious application for optically powered implants, due to the ease of light in-coupling. However, transducers based on photovoltaics operated in the tissue transparency region (as discussed in Section 4.2.4) have been demonstrated in stimulators for peripheral nerves, spinal cord, or for powering of pacemakers. ${ }^{117-121}$

Typically, inorganic solar cells based on p-i-n silicon or gallium arsenide have been used to power medical implants because of their action spectrum overlap with the tissue transparency window and their high power conversion efficiencies up to $45 \%$. ${ }^{117,118,122}$ However, relatively thick material layers are necessary to absorb the light efficiently, thus the devices are quite bulky and rigid. Inorganic devices can be thinned down to about $6 \mu \mathrm{m}$-thick absorption layers on flexible substrates and retain $21.7 \%$ power conversion efficiency. ${ }^{121}$ Nevertheless, a few micrometers of active material remains a hard limit, otherwise the power conversion efficiencies drop dramatically. ${ }^{123}$ The advancement of organic photovoltaics allows for ultrathin, light-weight, conformable devices, which can be advantageous for implanted or skin electronics. ${ }^{124}$ The efficiency and stability of organics remain lower in comparison to their 
inorganic counterparts; and thus the use of photovoltaics always presents a trade-off between efficiency and invasiveness and the choice of material will always depend on the specific application.

\subsubsection{Internal power transfer systems}

Energy can also be harvested from the internal processes in the body. ${ }^{5,125}$ The ambient energy can come from temperature differences using thermoelectric devices, from the biochemical environment by employing bio-fuel cells, or from movement with the use of piezoelectric devices and electrostatic, triboelectric, or electromagnetic generators. The concept of internal power source is very attractive, since there is no need for an external device to deliver the power, however, the amount of energy available from such devices is not sufficient for driving of most implantable devices and circuits. Therefore, these technologies remain nascent. 



\section{Methodology}

\subsection{Materials}

The most important organic electronic materials used in this thesis are briefly introduced in this section.

\subsubsection{PTCDI}

Perylene diimide derivatives are red colorants with excellent stability. Due to their high electron affinity and relatively high electron mobility they are

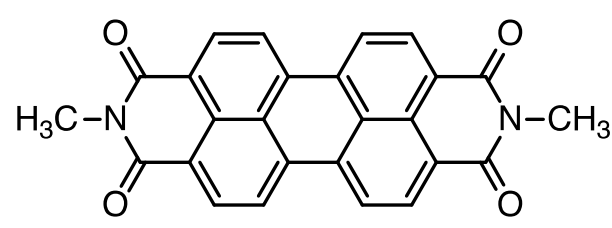
good acceptor materials for organic photovoltaics. ${ }^{3,126}$ The derivative $N, N$ '-dimethyl perylene tetracarboxylicdiimide (PTCDI) was used throughout this thesis.

\subsection{2. $\mathrm{H}_{2} \mathrm{Pc}$}

Phthalocyanines are macrocyclic aromatic organic molecules used as blue pigments. An absorption peak at $600-800 \mathrm{~nm}$ together with high absorption coefficient and semiconducting properties make phthalocyanines ideal for use as absorbers in solar cells in the biological tissue transparency window. They have been applied in donor-acceptor photovoltaic cells

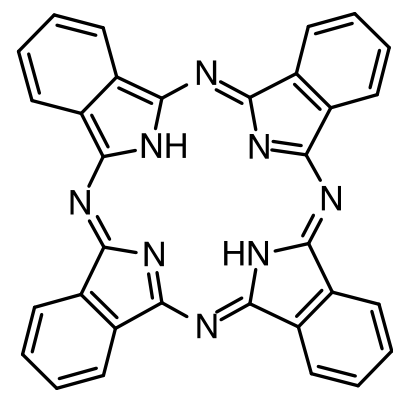
and in photodynamic therapy., ${ }^{3,127}$ In this thesis, metal free phthalocyanine $\left(\mathrm{H}_{2} \mathrm{Pc}\right)$ was used as the donor molecule in heterojunction photovoltaics and photocapacitors. $\mathrm{H}_{2} \mathrm{Pc}$ was chosen based on its stable interphase with PTCDI in biological conditions. ${ }^{7}$

\subsubsection{Epindolidione}

Epindolidione is an organic semiconducting pigment widely used as a yellow colorant. It exhibits ambipolar behavior in organic field effect transistors. ${ }^{128}$ In this thesis, it was used for<smiles>O=c1c2ccccc2[nH]c2c(=O)c3ccccc3[nH]c12</smiles> 
its fluorescence properties as an integrity check after a newly developed photolithography processing step.

\subsubsection{PEDOT:PSS}

Poly(3,4-ethylenedioxythiophene) doped/blended with polystyrene sulphonate (PEDOT:PSS) is an intrinsically conducting polymer system widely used in organic electronics for its chemical stability and high electronic and ionic conductivity. The conductivity of commercial PEDOT:PSS formulations can be increased by several orders of magnitude by additives such as dimethylsulfoxide or ethylene glycol which promote more ordered packing during drying. ${ }^{129}$ Due to its high volumetric capacitance, it is frequently used for stimulation and recording electrode coatings. ${ }^{54}$ PEDOT:PSS was used in Paper I to increase the capacitance of the indium tin oxide (ITO) back electrode and as a high injection capacity coating for the microelectrodes in Paper IV.

\subsubsection{PSS-Co-MA}

Poly(4-styrenesulfonic acid-co-maleic acid) (PSS-co-MA) crosslinked with polyethylene glycol was used as the cation exchange membrane in the OEIP fabrication. The membrane has a great ion selectivity and can be adopted in various processes. The recipe was developed by Arbring Sjöstrom et al. ${ }^{130}$ and has since been applied on a variety of surfaces and processing techniques. The PSS-co-MA is commercially available as a sodium salt, but the acid form is necessary for the crosslinking reaction. Thus, the polymer was first dialyzed in $\mathrm{HCl}$. Due to the acidic form of the membrane, it is necessary to develop the photoresist layer quickly in order to prevent the layer dissolution in the basic developer.

\subsection{Fabrication}

A number of fabrication techniques were used for the development of the devices described in this thesis. This section provides a brief theoretical background as well as some practical points for the processes employed.

\subsubsection{Physical vapor deposition}

Physical vapor deposition (PVD) is a thin film growth technique based on transferring of solid materials into vapor phase by physical means and their condensation on a substrate with no chemical change to the material 
(with the exception of reactive sputtering). The transition to the vapor phase can be done by resistive heating, electron-beam heating, or ion bombardment. The deposited layer thickness can be monitored by a quartz crystal microbalance (QCM) and controlled by a mechanical shutter placed below the substrate holder (Figure 6.1). Since the ratio of the material condensed on the samples and the monitor depends on the QCM position, it has to be determined experimentally for each sourcesubstrate-QCM geometry. The ratio is called the tooling factor:

$$
T F=\frac{\text { amount of material on the substrate }}{\text { amount of material on the QCM }}
$$

In systems with reproducible and constant deposition rates (sputtering and molecular beam epitaxy) the thickness is often controlled by the deposition time. To ensure layer uniformity, the substrate holder is usually rotating during the deposition. When using shadow masks for small-feature patterning, rotation is sometimes not applied to limit the shadowing effect. The thin film purity is provided by bringing the system to high vacuum $\left(10^{-6}\right.$ Torr $)$ before the deposition. An illustration of a PVD chamber is shown in Figure 6.1. The PVD techniques used in this thesis include thermal evaporation and sputtering. Thermal evaporation is limited to thermally stable materials capable of entering the vapor phase upon heating, while sputtering is possible for essentially all materials. 

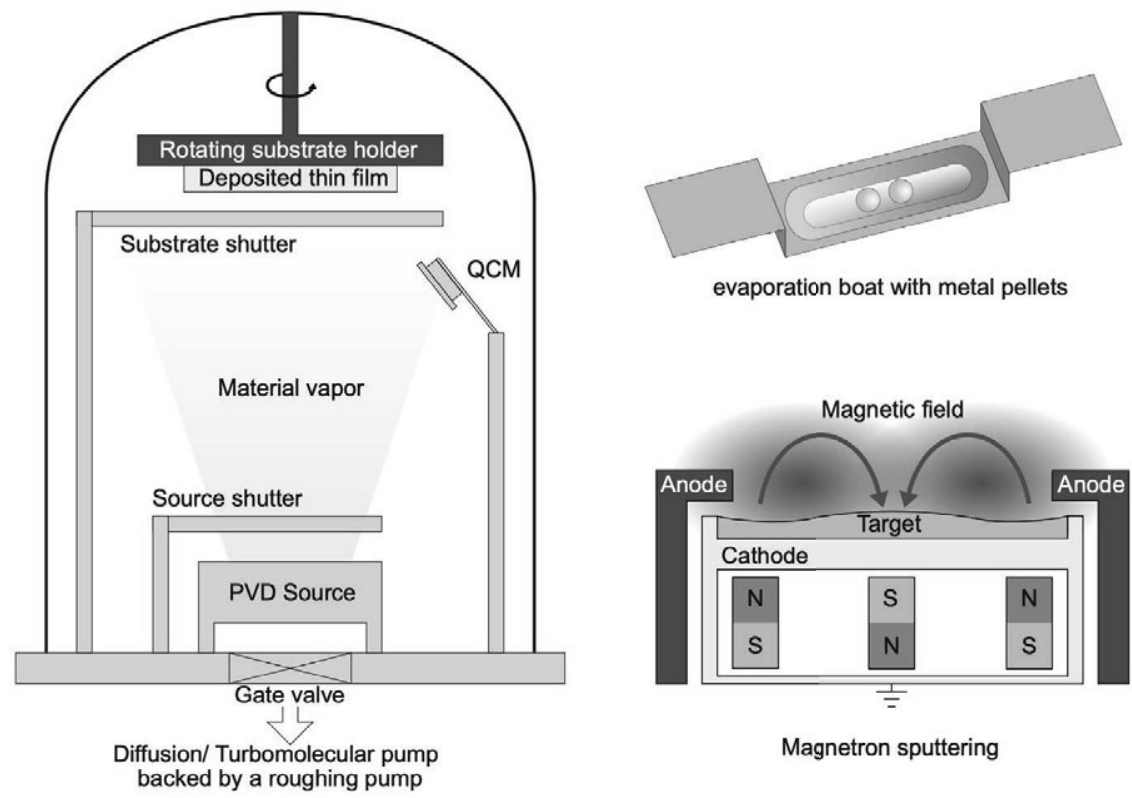

Figure 6.1: A general scheme of a PVD system, with the two types of sources: thermal evaporation boat and a magnetron sputtering gun.

\subsubsection{Thermal evaporation}

In thermal evaporation, the energy needed for the material to enter vapor phase is delivered by resistive heating. The vaporized molecules can collide with other gas molecules present in the system. To avoid such collisions, high vacuum is necessary. The molecular mean free path defines the distance a particle can travel before being deflected by another and is a function of pressure.

$$
m f p=\frac{k_{B} \cdot T}{\sqrt{2} \cdot \pi d^{2} \cdot p_{b}}
$$

Where $k_{B}$ is the Boltzmann constant $\left(1.38 \cdot 10^{-23} \mathrm{~J} / \mathrm{K}\right), \quad T$ the gas temperature, $\pi d^{2}$ the effective collision area for 2 molecules of diameter $d$, and $p_{b}$ the background pressure.

From the perspective of mean free path, most thermal evaporation systems require pressures lower than $10^{-6}$ Torr to allow the material to travel in a straight line from the source to the substrate. Due to the 
directional deposition, thermal evaporation is prone to shadowing effects on substrates with high vertical feature size. If film contamination is an issue, high vacuum $\left(10^{-7}\right.$ Torr $)$ or ultrahigh vacuum $\left(10^{-9}\right.$ Torr $)$ must be employed. Our go-to base pressure is $<2 \cdot 10^{-6}$ Torr for most applications, where the mean free path is on the order of meters and surface contamination is limited. Film purity can be further increased by employing high deposition rates to increase the partial pressure of the material of interest.

\section{Metals}

Most metals enter vapor phase by evaporation, but some do it by sublimation $(\mathrm{Cr})$ or the combination of evaporation and sublimation (Ti). ${ }^{131}$ Ultrapure metals can be purchased as pellets, wire, or powder. The heating source comes in a variety of forms, from wires, crucibles, and baskets, to thin boats made of high melting-point metals such as W, Mo, or Ta. For metal evaporation, boats are the most common choice (Figure 6.1) in a laboratory setting. By passing current through the boat, it gets resistively heated thus supplying the heat to the material. The fixture of the boat to the source should not be too tight to accommodate thermal expansion and prevent stress related breaking. Some materials can react with the underlying boat, in these cases the deposition should be done as fast as possible. Typical problematic combinations are $\mathrm{W}$ with $\mathrm{Al}$, Ti, or Pd. ${ }^{131}$ Good wetting of the heating source by the desired material is crucial for a good evaporation behavior. Aluminum is notorious for its alloying with tungsten when molten, leading to fast boat degradation and source contamination. This phenomenon can be limited by the use of $\mathrm{W}$ wire clippings along with the $\mathrm{Al}$ source, the clippings serve for the aluminum wetting and provide better controlled Al evaporation and higher deposition rates. This approach was used when making thick $\sim 2 \mu \mathrm{m}$ aluminum layers. The heated source releases radiative heating to the whole chamber causing all of the surfaces to heat up. In order to limit the temperature impact on the samples, the evaporation should be done rapidly. Depending on the material, deposition rates can be between $0.1 \AA / \mathrm{s}$ to hundreds of $\mathrm{nm} / \mathrm{s}$.

\section{Organics}

Thermal evaporation of organics is limited to thermally stable materials of moderate molecular weight $(\leq 700 \mathrm{Da})$ that can sublime without degradation. Generally, only small molecules can be processed this way, since most polymers - with the exception of polyethylene - decompose 
upon heating. ${ }^{132}$ Vacuum deposition of organics is normally done from tungsten wire heated crucibles made of alumina or carbon. Direct contact to the metal heating source can cause organic material carbonization thus leading to contamination and should be avoided. Ultra-pure materials are necessary in order to grow high-quality thin films. Purity is especially important for PVD of organics, since the commercially available powders typically contain large amounts of impurities from the synthesis. The materials used in this thesis were purified by thermal gradient sublimation. The technique is based on the difference of the heat of condensation of the pure material and the impurities. The substance is loaded into a tube which is heated using a furnace. The purified crystals condense in a specific section of the thermal gradient. ${ }^{133}$ The procedure is typically repeated three times before use in PVD. Organic evaporators are more prone to cross-contamination, therefore, the chamber should be cleaned before changing to a different material. In general, organic evaporators should be cleaned more often than metal evaporators due to the spongy high surface area build up that is very good at adsorbing outgassing molecules like water vapor. Further, the crucibles should be preheated before each deposition to remove the volatiles from the organic crystals. Typical deposition rates are between 0.1 to $10 \AA / \mathrm{s}$. The morphology of organic evaporated films can be controlled by the deposition rate and substrate temperature.

\subsubsection{Sputtering}

In sputtering, the transition to vapor phase is done by momentum transfer during ion bombardment. Argon atoms are ionized in plasma and are accelerated towards the negatively biased target (cathode) thereby ejecting atoms of the target material. As a plasma process, sputtering requires a working pressure of argon in the range of 1-100 mTorr. Therefore, the mean free path is shorter in sputter deposition. It provides the benefit of being able to conformally coat samples with complex vertical features that would normally be shadowed during a thermal deposition. Sputtering is often done in the magnetron gun configuration (Figure 6.1), which uses magnetic fields to circulate electrons above the target surface thus producing a high-density plasma. Highly conductive materials can be deposited via direct current sputtering. However, deposition of low conductivity materials and dielectrics can be done by radio frequency sputtering. In RF sputtering, the applied potential is alternating typically at $13.56 \mathrm{MHz}$. During one half cycle, the ions are accelerated toward the target causing sputtering, while during the other 
half cycle the target surface is neutralized to prevent charge buildup. As a general rule, RF sputtering proceeds at low deposition rates. The number of target atoms released per $\mathrm{Ar}^{+}$collision is defined as the sputtering yield. It can range from $0.5-4$ for metals. ${ }^{134}$ Sputtering yield is one of the variables affecting the deposition rate. Compound films can be formed by sputtering from a compound target and/or by reactive sputtering. Typically, oxides and nitrides are made by reactive sputtering in partial pressure of oxygen or nitrogen respectively. The target is actively cooled in most systems, so radiative heating inside of the system is limited. Therefore, sputtering may be better suited for temperaturesensitive substrates rather than thermal evaporation. Sputtering pressure affects the thin film properties such as the film stress and morphology. High pressure leads to tensile stress and rougher films, low pressure yields more uniform films with compressive stress. ${ }^{131}$ The other important parameters are the gas flow, target power, and the gas composition. The biggest advantage of sputtering is the ability of most materials - including dielectrics - to be sputtered. However, sputtering is generally slower and less energy efficient than thermal evaporation. Targets can be fragile and very expensive, thus making it a big investment when new material is introduced into the system. Once a target is installed, however, the materials usage is more efficient than thermal evaporation, making sputtering usually favored in industrial processes.

\subsubsection{Chemical vapor deposition}

Chemical vapor deposition (CVD) refers to a group of techniques in which a thin film is grown from vapor phase. In contrast to PVD, a chemical reaction has to occur on the substrate surface. The thin film properties can be altered by changing the temperature, gas flow and precursor composition. CVD processes can take place in a variety of chambers ranging from simple Petri dishes to sophisticated plasma/ vacuum systems. The following materials were processed via CVD in this thesis.

\subsubsection{Parylene}

Parylene or poly(para-xylylene) comprises a group of CVD-grown hydrophobic semicrystalline polymers used widely in industry for their conformal, pinhole-free nature and good barrier properties. ${ }^{135}$ The basic polymer poly(para-xylylene), commercially known as parylene $\mathrm{N}$, has many substituted derivatives. The chlorinated version parylene $\mathrm{C}$ has 
become the favorite in the fields of bioelectronics and microfabrication ${ }^{136}$ due to its excellent barrier properties, high deposition rate, chemical stability and proven biocompatibility. Parylene $\mathrm{C}$ is typically used as a substrate and encapsulation, but it can also be used for patterning, which is discussed in a further section. The parylene precursor is a stable dimer, which is pyrolyzed into two radical monomer molecules that spontaneously polymerize at room temperature (Figure 6.2). The polymer is grown via radical polymerization in vacuum which allows for high molecular weight films. A typical parylene CVD system consists of a dimer vaporizer, cracking furnace, deposition chamber, vacuum pump, and a cold trap to prevent the reactive monomer from entering the pump. The film thickness can be controlled by the dimer loading weight or by following the deposition pressure and time in systems equipped with a gate valve between the vaporizer and the cracking oven. The resulting thin films can be patterned by photolithography and reactive ion etching. Parylene $\mathrm{C}$ has a very low surface energy, therefore the adhesion between two parylene $\mathrm{C}$ layers is very limited. For that reason, adhesion promoters are typically used between the substrate and encapsulation layers. A silane with a methacryloyl group is most often used, however other substances such as propylene carbonate can also enhance adhesion. ${ }^{137}$

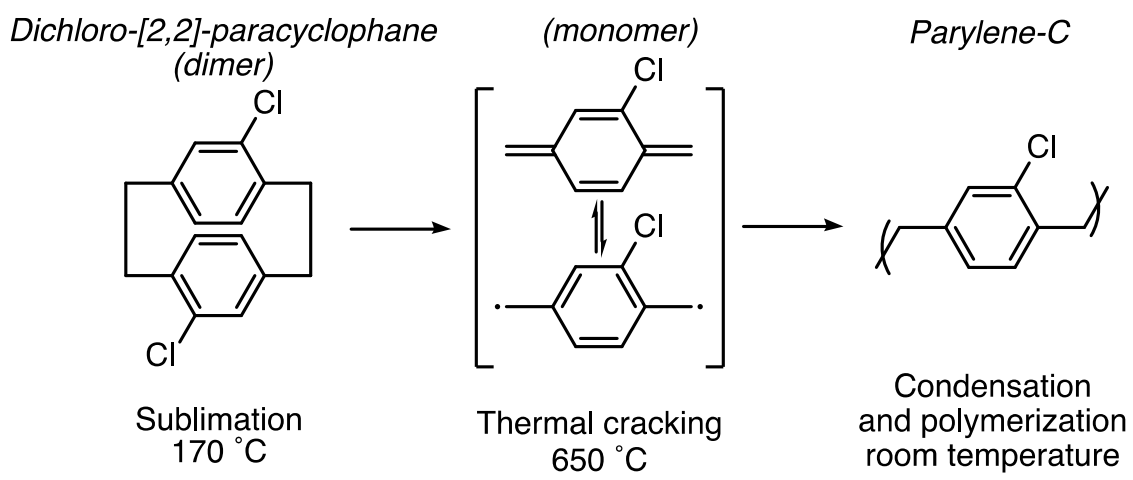

Figure 6.2: The polymerization scheme for parylene C. 


\subsubsection{2. $\operatorname{SiN} x$}

Silicon nitride is a hard, ceramic material used as a dielectric or for passivation in (bio)electronics for its chemical and thermal stability and electrical resistivity. It is also widely used as an etch mask in silicon microfabrication. Silicon nitride films are typically grown by chemical vapor deposition. Conventionally, highly crystalline $\mathrm{Si}_{3} \mathrm{~N}_{4}$ layers are grown via low pressure (LP)CVD that requires high temperatures between $1000-1700{ }^{\circ} \mathrm{C}$. However, many microfabrication processes are not compatible with such conditions. Therefore, plasma enhanced (PE)CVD to produce $\mathrm{SiN}_{\mathrm{x}}$ at lower temperatures has gained much attention. In PECVD, the high activation energy for the reaction is delivered by a plasma process instead of high temperature. This process allows $\mathrm{SiN}_{\mathrm{x}}$ growth in the range from room temperature to $350{ }^{\circ} \mathrm{C} .{ }^{138}$ However, unlike LPCVD, PECVD does not produce stoichiometric oxides and nitrides. ${ }^{134}$ Silane $\left(\mathrm{SiH}_{4}\right)$ and ammonia $\left(\mathrm{NH}_{3}\right)$ with nitrogen $\left(\mathrm{N}_{2}\right)$ are the most typical precursors, but others have been reported. ${ }^{139}$ Temperature, pressure, flow rate and flow rate ratio are the main factors influencing the deposition rate and film properties in thermal CVD, while PECVD has an additional variable in the form of RF power. A typical configuration of a PECVD deposition system is illustrated in Figure 6.3.

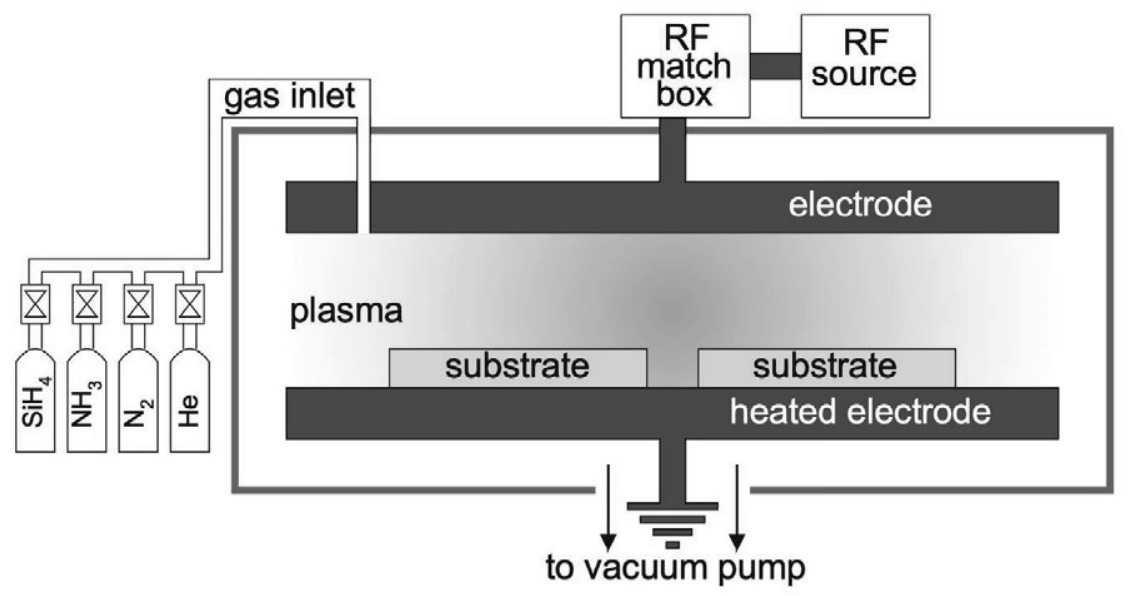

Figure 6.3: A capacitively coupled parallel plate PECVD system. The impedance matching of the source and load to maximize the transferred power is achieved by a RF matching network (RF match box). The substrates are normally heated to $350{ }^{\circ} \mathrm{C}$ during the deposition. 


\subsubsection{Silanization}

Organosilanes are agents used for functionalization of a variety of surfaces with available hydroxyl groups. ${ }^{140}$ The molecules are of the general structure $\mathrm{R}-\mathrm{Si}-\mathrm{X}_{3}$ with $\mathrm{R}$ being a functional group and $\mathrm{X}$ a leaving group. The surface hydroxyl groups carry out a nucleophilic attack on the silane molecule thus displacing one of the leaving groups. The remaining leaving groups crosslink with neighbouring hydroxyl groups or silane molecules thereby producing a self-assembled monolayer. The surface properties are then dictated by the silane functional group. An illustration of a silanization reaction along with examples are demonstrated in Figure 6.4. Octyltriethoxysilane (OTS) was used to form hydrophobic surfaces on indium tin oxide (ITO) to prevent delamination of vacuum deposited small molecules. (3-Mercaptopropyl)triethoxysilane (MPTMS) served as an adhesion promoter for gold layers. (3-Glycidyloxypropyl)trimethoxysilane (GOPS) was used for covalent bonding between the silanized surface and polymers with available hydroxyl groups, such as polystyrene sulfonate (PSS). GOPS further served as a crosslinker in polymer formulations containing PSS. ${ }^{141}$ 3-(Trimethoxysilyl)propyl methacrylate (A-174) acted as an adhesion promoter for films grown by radical polymerization, such as the parylene CVD. Trichloro( $1 H, 1 H, 2 H, 2 H$-perfluorooctyl)silane (F-OTS) was used to create omniphobic surfaces.

The reactions can be done either in solution or in vapor phase. The liquid phase process typically yields multilayers, requires optimization of the solvent and other conditions, and requires large solution volumes. ${ }^{142} \mathrm{On}$ the contrary, vapor phase deposition normally produces true monolayers, because the oligomerized silane reagent cannot enter the vapor phase. To increase the silane partial pressure, it is either heated in a closed compartment together with the substrate or by lowering the base pressure of the system. The temperature required for the CVD process decreases with the increasing reactivity of the silane leaving group: ethoxy $<$ methoxy $<$ chloro. The reaction byproducts are defined by the nature of the leaving group and should be considered during process design to be compatible with the underlying structures. A controlled nitrogen atmosphere yields monolayers of superior quality. Some silanes can form multilayers in CVD due to non-covalent interactions of the functional groups. Such samples can be sonicated after the deposition to release the physisorbed molecules. Most silanizations in this thesis were performed in stainless-steel Petri dishes with $70 \mu \mathrm{L}$ of the silane spread 
around. The Petri dish was heated to $70-90{ }^{\circ} \mathrm{C}$ using a hot plate. F-OTS reacts efficiently already at room temperature and A-174 treatment was done directly in the parylene CVD chamber just before parylene deposition.
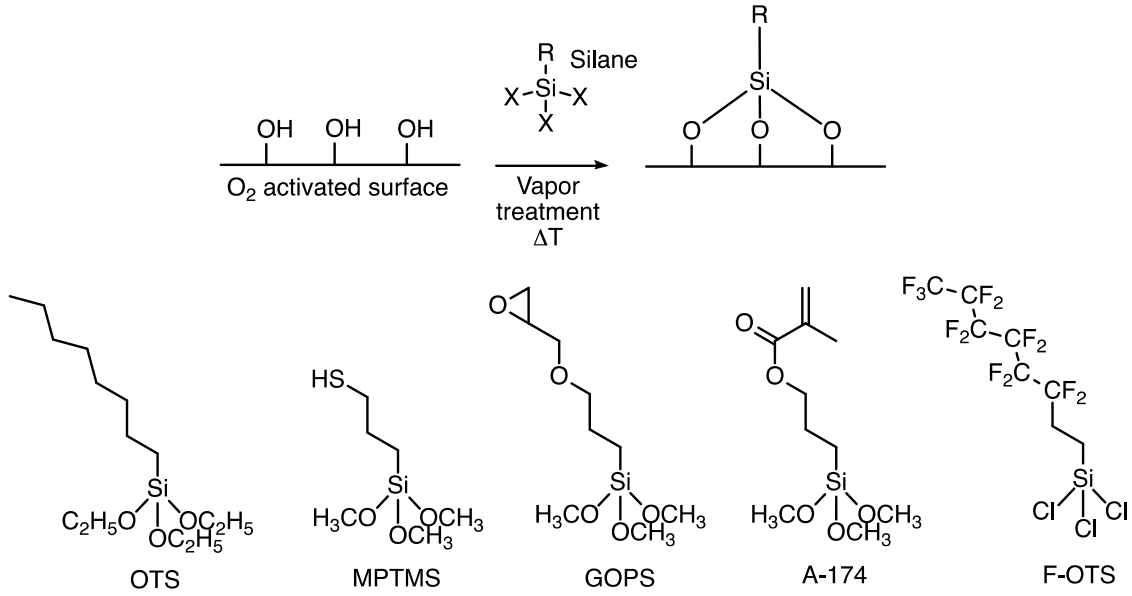

Figure 6.4: General scheme of silanization vapor treatment and the structures of silanes used in the scope of this thesis.

\subsubsection{Solution based deposition}

Solution based techniques are very common in the field of organic electronics, because a large portion of the materials are soluble in various solvents. This allows for the application of techniques like printing, dip coating, bar coating, spin coating, spray coating and a variety of others. The techniques do not require vacuum technology and the material usage can be very efficient (inkjet printing, drop casting). A problem of most solution-based deposition techniques is the necessity of a flat substrate. If the sample contains structures of a high aspect ratio, the coatings are inherently not uniform. Within this thesis, spin coating and drop casting were the only methods applied.

\subsubsection{Spin coating}

In spin coating, a substrate is mounted on a vacuum chuck, the material solution is dispensed in the middle of the substrate, which is then spread using centrifugal force. ${ }^{143}$ The spinning usually has two steps: the first, slower step serves for spreading the material homogenously over the whole substrate surface; the second is used for removing the excess 
material and drying off the solvent to form the final thin film (Figure 6.5). Closed spin coater systems with saturated solvent vapor to ensure slow solvent evaporation are often necessary for homogeneous spread of thick films. The parameters influencing the thickness of a spin coated film are the material concentration, viscosity and the spin speed. ${ }^{144}$ Due to its high reproducibility, spin casting is widely used in lithography for photoresist coating. It is also one of the most common methods for the deposition of solution based organic electronic materials. Some of the disadvantages are poor material usage efficiency and limitation to small substrates. Spin coating is best suited to round substrates as rectangles usually show corner effects.

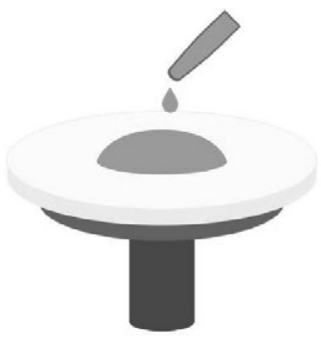

Material deposition

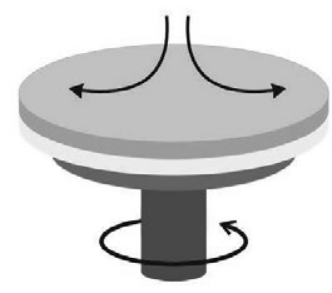

Spread step

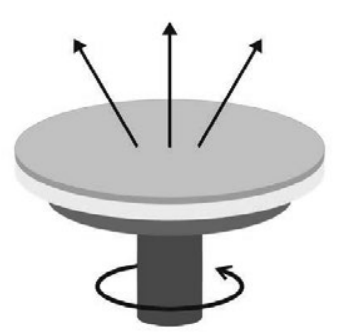

Spin \& dry

Figure 6.5: Procedure scheme for spin-coating, proceeding from left to right.

\subsubsection{Patterning techniques}

Pattern formation is a vital step during fabrication of multilayer devices. The most developed technique used in the production of semiconductor technology is photolithography due to its high resolution, scalability, reproducibility, and applicability to a wide range of inorganic materials. Conventional photolithography processing includes exposure to organic solvents, resists and developers which are generally not compatible with organic materials. Therefore, alternative methods are required in processing of organics. Generally, printing techniques are used to pattern solution-based organic materials, while stencil masking is the method of choice for PVD materials. Both approaches have significantly lower resolution than photolithography and thus significant effort is invested in developing photolithographic processes compatible with organic materials. One approach is using fluorous solvents and developers orthogonal to the organic materials' chemistry. ${ }^{145,146}$ Alternatively, various kinds of protective layers can be used during a subtractive or 
additive patterning step. ${ }^{14-149}$ In the following section, the patterning techniques used within this thesis are discussed, noting the specifics of organics patterning.

\subsubsection{Shadow masking}

The simplest and fastest way to pattern PVD materials is by using a shadow mask. A shadow mask is a perforated flat metal or plastic foil placed in close proximity with the substrate during the deposition. The apertures form the pattern that will be translated onto the substrate. The resolution of the technique is dictated by the thickness of the shadow mask and the distance between the substrate and the mask (Figure 6.6). In typical lab-scale conditions, the minimum-feature size is approximately $50 \mu \mathrm{m}$. When using precision shadow masks one can lower the limit to $\sim 5 \mu \mathrm{m}$. ${ }^{150,151}$ The main limitation comes from the fact that the shadow mask has to be made of a single piece of material, therefore some designs are inherently impossible, i.e. concentric openings. Further, masks with high density of apertures are challenging to produce and are very fragile. Thermal expansion during evaporation can cause structure misalignment and wider shadow effect due to separation of the mask from the substrate. ${ }^{151}$ The devices presented in this thesis were patterned using a $75 \mu \mathrm{m}$ thick laser patterned stainless steel masks or custom-cut PVC masking tape.

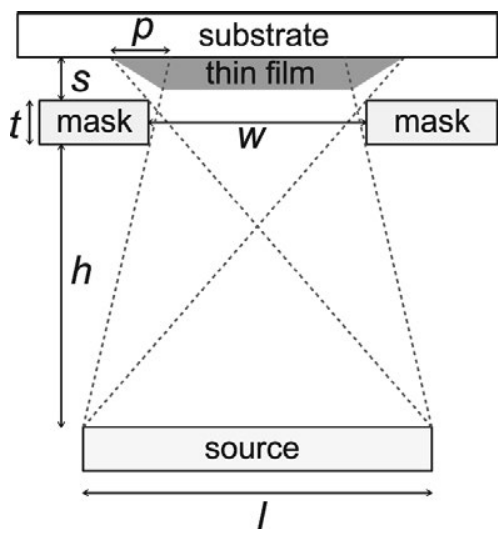

Figure 6.6: Schematics of PVD patterning using a shadow mask. The shadow effect dictates the resolution of the technique. The taper edge of the thin film depends on the source size $l$, distance of the source from the mask $h$, mask thickness $t$, distance between the mask and the substrate $s$, and the size of the aperture $w$. Figure adapted from [150]. 


\subsubsection{Photolithography}

Lithography is a widely used technique based on patterned resist films to define thin film structures. The resist patterning can be done either by exposure to light (photolithography) or to electron beam (E-beam lithography). A typical workflow in photolithography includes:

1) photoresist coating

2) mask alignment

3) UV light exposure

4) development

5) thin film etching

6) photoresist stripping

Photoresists are materials whose solubility changes by the exposure to UV light. ${ }^{152}$ Light exposure solubilizes positive tone resists, thereby transferring the photomask pattern onto the substrate. Negative tone photoresists are crosslinked by UV light and thus the pattern on the substrate is the negative image of the mask (Figure 6.7). Photoresists are typically applied by spin coating, but lamination or spray coating can sometimes be superior. The pattern is given by a photomask design. Photolithography masks can be made of patterned transparent foils either by inkjet printing or laser photoplotting $(>10 \mu \mathrm{m})$, while high resolution masks are chromium patterns on glass $(>3 \mu \mathrm{m})$ or quartz $(<3 \mu \mathrm{m})$ patterned by laser or E-beam lithography. ${ }^{134}$ Plastic masks are cheaper and faster to fabricate but suffer from lower mechanical stability, less precise alignment and can cause lower quality of negative photoresist layers due to inhomogeneities of the mask film. Mask alignment and light exposure are done in a mask aligner tool, which allows precise positioning of the substrate in relation to the mask and provides a largearea, homogeneous, calibrated light source. The alternative to mask aligners are maskless projection or direct writer systems ${ }^{153}$, which have lower throughput, but are advantageous for prototyping. After exposure, the soluble part of the photoresist is removed in a developer solution producing the final pattern. Positive resists are mostly developed in basic aqueous conditions, while most negative resists require organic solvents as developers. In the next step the underlying thin film is etched away. Finally, the photoresist has to be removed either by dissolving in acetone, a stripping agent, or by oxygen plasma etching. An alternative use of photolithography is the lift-off procedure, widely used for patterning of metals. Here, the photoresist is patterned first, then the thin film is 
deposited and the photoresist along with the excess material is "liftedoff" in acetone or stripping solution producing a thin film negative image of the photoresist pattern.

Photolithography is a very powerful technique with better resolution and minimum feature size than shadow masking. It is very reproducible, easy to align multilayer structures, and it allows any kind of arbitrary design. However, the materials processed by lithography have to be compatible with the workflow including relatively high temperatures and organic solvents. An alternative photolithography method to pattern organic electronic materials is described in Paper II. It is based on double exposure of a positive photoresist. The first exposure and development provide the pattern, while the second exposure turns the residual resist soluble in basic potassium hydroxide developer. Therefore the technique could be described as an aqueous lift-off. ${ }^{154}$

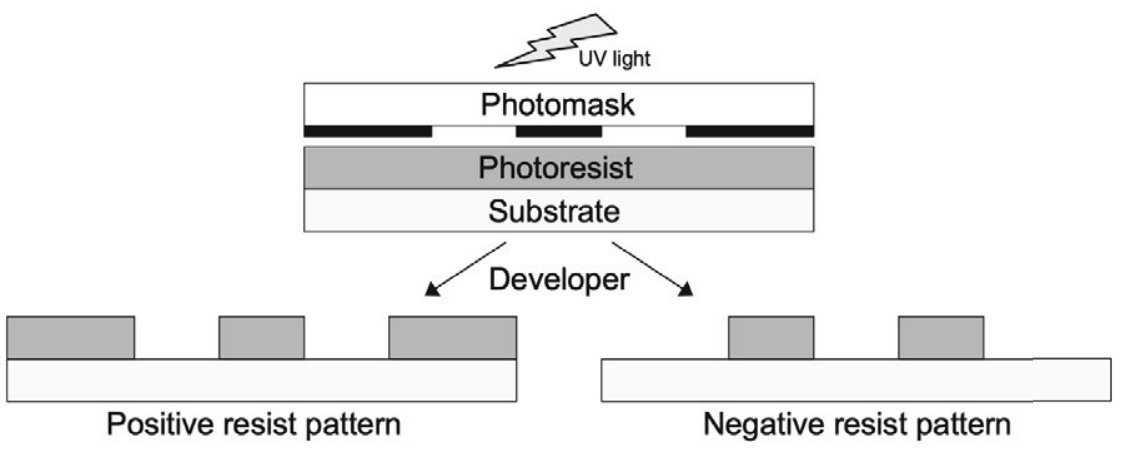

Figure 6.7: Photolithography scheme.

\subsubsection{Parylene peel-off}

Conceptually, the parylene peel-off technique is a form of ultra-precise shadow masking or dry lift-off. The substrate is coated with an antiadhesion layer (typically a dilute solution of soap) and a thin film of parylene $\mathrm{C}$ via CVD. The openings are patterned by lithography and reactive ion etching, thereby producing a conformal ultrathin polymer shadow mask in direct contact with the substrate. Afterwards, the layer of interest is applied and the parylene layer is peeled off yielding the desired pattern (Figure 6.8). The technique was first developed to pattern solution-based biological species on top of various surfaces. ${ }^{155}$ The dry lift-off nature of the method was essential, since it retains the resolution and fidelity of conventional photolithography, but omits the contact with 
organic solvents harmful to the sensitive biomolecules or cells. The method was later adopted in patterning of solution-based electronic and other functional materials on more complex substrates. ${ }^{149,156,157}$ The main disadvantage of the technique is the necessity of an etch stop for the RIE patterning step. Therefore, this technique cannot be easily used for patterning on top of sensitive substrates. Further, the method is not well suited for patterning by wet chemical etching, because the contact with water causes partial delamination of the edges leading to an undercut. A parylene peel-off sequence was developed for patterning of the thermally evaporated organic thin film on top of a sensitive $10 \mathrm{~nm} \mathrm{Au}$ layer on parylene C in Paper III.
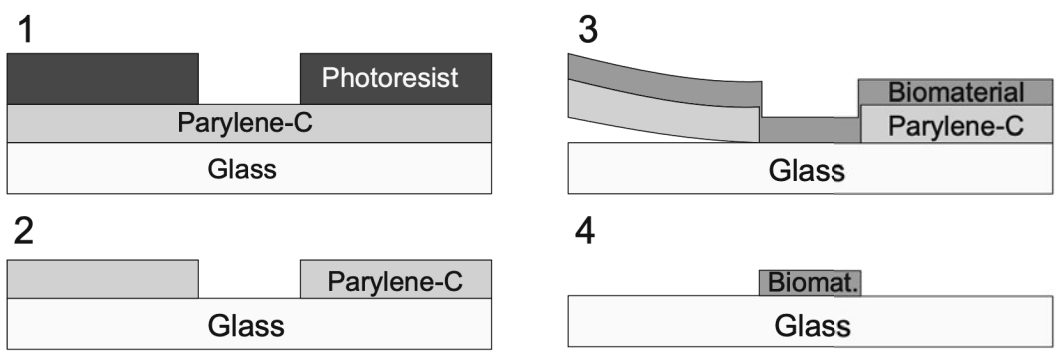

Figure 6.8: Workflow of parylene peel-off patterning. 1) parylene $\mathrm{C}$ deposition and photoresist patterning, 2) RIE and photoresist stripping, 3) deposition of biomaterial and parylene $\mathrm{C}$ peel-off, 4) patterned biomaterial.

\subsubsection{Etching}

Lithography is only one part of the patterning process; the material thin film has to be etched away in the exposed regions in order to transfer the pattern. Etching is based on a chemical reaction between the thin film and the etchant reactive species. There are two types of etching: wet etching involves reaction in liquid etchant producing soluble products, while dry (or plasma) etching is based on gaseous etchant producing volatile products. $^{134}$

\subsubsection{Wet etching}

Wet etching is typically done in an etchant bath, which can be homogeneously heated. The container has to be made of an inert material that does not react with the etching solution. Glass or polypropylene beakers are suitable in most cases, quartz and Teflon are used otherwise. Most wet etching is based on redox or acid-base reactions. Wet etching 
is in most cases isotropic and leads to an undercut on the order of the film thickness. The compositions of the etchants used within this thesis are given in Table 6.1.

Table 6.1: Wet etchant recipes

\begin{tabular}{lll}
\hline Material & Etching solution & Fraction type \\
\hline $\mathrm{Au}$ & $\mathrm{KI}: \mathrm{I}_{2}: \mathrm{H}_{2} \mathrm{O}(4: 1: 400)$ & $\mathrm{w} / \mathrm{w} / \mathrm{w}$ \\
$\mathrm{Pd}$ & $\mathrm{KI}: \mathrm{I}_{2}: \mathrm{H}_{2} \mathrm{O}(10: 1: 40)$ & $\mathrm{w} / \mathrm{w} / \mathrm{w}$ \\
$\mathrm{ITO}$ & $\mathrm{HCl}$ conc. & \\
$\mathrm{Al}$ & $\mathrm{H}_{3} \mathrm{PO}_{4}: \mathrm{HNO}_{3}: \mathrm{H}_{2} \mathrm{O}(80: 4: 16)$ & $\mathrm{v} / \mathrm{v} / \mathrm{v}$ \\
$\mathrm{Ti}$ & $\mathrm{NH}_{3}: \mathrm{H}_{2} \mathrm{O}: \mathrm{H}_{2} \mathrm{O}_{2}(2: 1: 1)$ & $\mathrm{v} / \mathrm{v}$ \\
& $\mathrm{HF}: \mathrm{H}_{2} \mathrm{O}_{2}: \mathrm{H}_{2} \mathrm{O}(1: 1: 10)$ & $\mathrm{v} / \mathrm{v} / \mathrm{v}$ \\
\hline
\end{tabular}

\subsubsection{Dry etching}

Dry etching is done in a vacuum chamber equipped with a plasma generator. Reactive ion etching (RIE), a form of dry etching, uses a system similar to PE-CVD with etching gasses creating the plasma of excited and ionized species. In RIE, chemical reactions and ion bombardment work synergistically to remove the thin film. In RIE, the RF field is applied between the substrate bottom electrode and the ground top electrode giving the correct polarity of the electric field to accelerate the positive reactive ions towards the substrate. Due to the parallel plate design, the applied electric field is perpendicular to the substrate thus producing highly anisotropic vertical etch. However, the anisotropy can be tuned by the process pressure, substrate temperature and the RF power. In this thesis, parylene $\mathrm{C}$ and OEIP ion channel patterning was done with $\mathrm{O}_{2}$ or $\mathrm{O}_{2} / \mathrm{CF}_{4}$ RIE. 


\subsubsection{Fabrication schemes for Papers III, IV, and V}

The devices developed in Paper III, IV, and V are examples of a complex multi-step fabrication including most of the methods mentioned above. The workflow is presented in Figure 6.9.

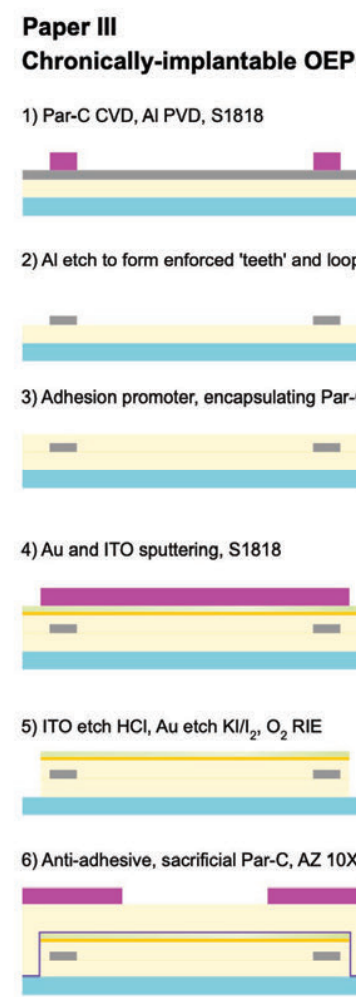

7) $\mathrm{O}_{2} \mathrm{RIE}$, ITO etch $\mathrm{HCl}$
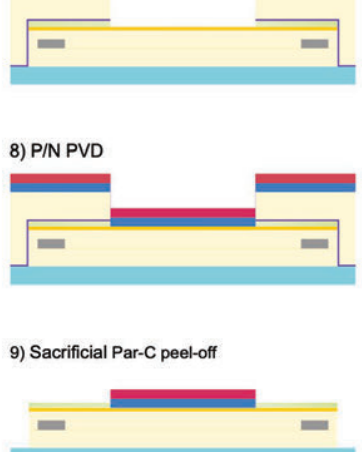

\section{Paper IV} OPV driven microstimulator

1) Par-C CVD, Pd/Au deposition, $S 1818$

$$
\text { ( }
$$

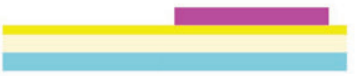

2) $A Z$ nLOF 2020, thin Au deposition

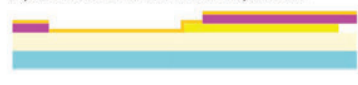

3) Lift-off, thin Au pattern

4) P/N PVD through a shadow mask

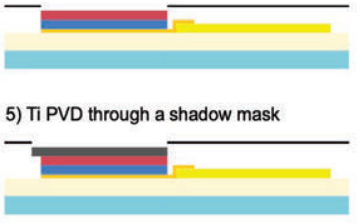

6) Adhesion promoter, encapsulating Par-C

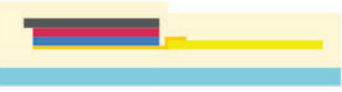

7) $A Z 10 X T, O_{2} / \mathrm{CF}_{4}$ RIE outline patterning

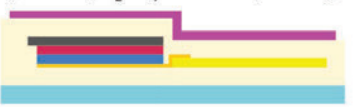

8) Anti-adhesive, sacrificial Par-C, AZ 10XT

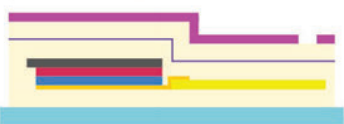

9) $\mathrm{O}_{2} / \mathrm{CF}_{4} \mathrm{RIE}$, PEDOT:PSS spin coating

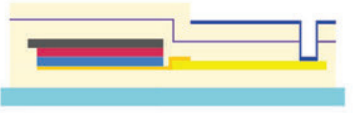

10) Sacrificial Par-C peel-off

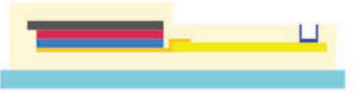

\section{Paper V \\ OPV driven OEIP}

1) Par-C CVD, PSS-co-MA, PMMA, $S 1818$

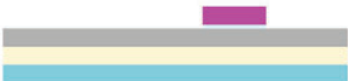

2) $\mathrm{O}_{2} / \mathrm{CF}_{4}$ RIE to define the ion channel

ral

3) Thin Au deposition, $\$ 1805$

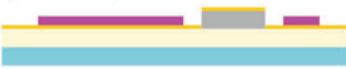

4) Au etch $K I / I_{2}$

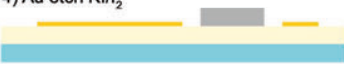

5) P/N PVD through a shadow mask
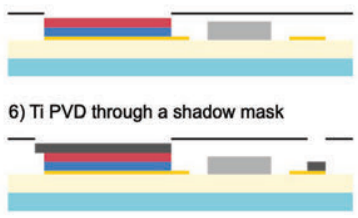

7) Adhesion promoter, encapsulating Par-C

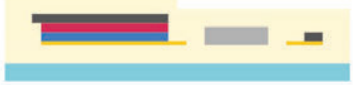

8) S1818, Al PVD to form a hard mask

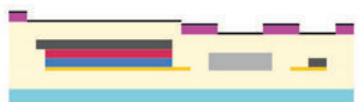

9) Al lift-off

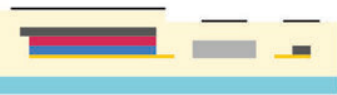

10) $\mathrm{O}_{2}$ RIE, Al etch

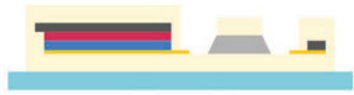

11) $\mathrm{Ag} / \mathrm{AgCl}$ paste

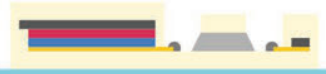

Figure 6.9: The fabrication workflow for Papers III, IV, and V. 


\subsection{Characterization}

The devices developed within the thesis were characterized with a number of optical, electronic, and biological techniques. Brightfield light microscopy was used continuously throughout the different fabrication steps to discover obvious failures or misalignments.

\subsubsection{Electrical characterization}

Since all of the bioelectronic devices are based on electronic or optoelectronic control, studying the electrical properties was the most basic form of characterization. The performance of photovoltaics is measured by its $\mathrm{I}(V)$ characteristics. The organic electronic ion pumps are ionic resistors; therefore, their behavior can be studied by measuring their steady state currents as a function of the applied voltage. The channel selectivity is probed by changing the transported ion and its concentration in the source electrolyte or by changing the co-ion in the target electrolyte.

\subsubsection{Chemical delivery characterization}

The efficiency of the specific ion delivery using the OEIP can be calculated by measuring the current passed through the circuit and the resulting number of ions delivered in the target. The quantification is not straightforward in the case of metal ions. On the contrary, many molecules can be detected via biological assays. The two most used techniques are enzymatic assays and enzyme-linked immunosorbent assays (ELISA). ELISA can be developed for virtually any organic analyte, because it is based on antibody recognition. Enzymatic assays are limited to analytes undergoing enzymatic reactions with a redox intermediate that can be visualized by an optical method or through electrochemical detection. Most optical enzymatic assays are based on one of the reactions producing hydrogen peroxide byproduct, which can be quantified by reaction with horseradish peroxidase (HRP) and a signal forming substrate. The readout can be absorbance, fluorescence, or luminescence. The molecule quantified within this thesis was acetylcholine. Acetylcholine reacts with acetylcholine esterase to form choline, which reacts further with choline oxidase, producing $\mathrm{H}_{2} \mathrm{O}_{2}$ as a side product. The peroxide concentration was then determined by reaction with HRP and 3,3',5,5'-tetramethylbenzidine (TMB) producing a blue product quantifiable at $653 \mathrm{~nm} .{ }^{158}$ The colorimetric analysis was 
performed using a plate reader. Since TMB is not stable in the buffer conditions of acetylcholine esterase and choline oxidase, the reaction was done in two steps with a buffer exchange before the HRP/TMB step.

\subsubsection{Electrophotoresponse}

The electrophotoresponse (EPR) measurement was used to characterize the organic electrolytic photocapacitor devices. ${ }^{7}$ The technique allows the determination of the maximum photovoltage and photocurrent the device can deliver. The method is used for batch-to-batch quality control, for characterizing new material combinations and device concepts, as well as for studying the performance stability. The measurement is done using an oscilloscope and bottom illumination with a red $630 \mathrm{~nm}$ light-emitting diode. The back electrode is contacted directly with a probe, while the illuminated part of the organic pixel is contacted electrolytically with a syringe electrode containing an $\mathrm{Ag} / \mathrm{AgCl}$ wire and $100 \mathrm{mM} \mathrm{KCl}$ (Figure 6.10). The photovoltage is measured directly by the oscilloscope, while the current is obtained by passing the signal through a low impedance current amplifier. The measurement setup is placed in a dark faraday cage.
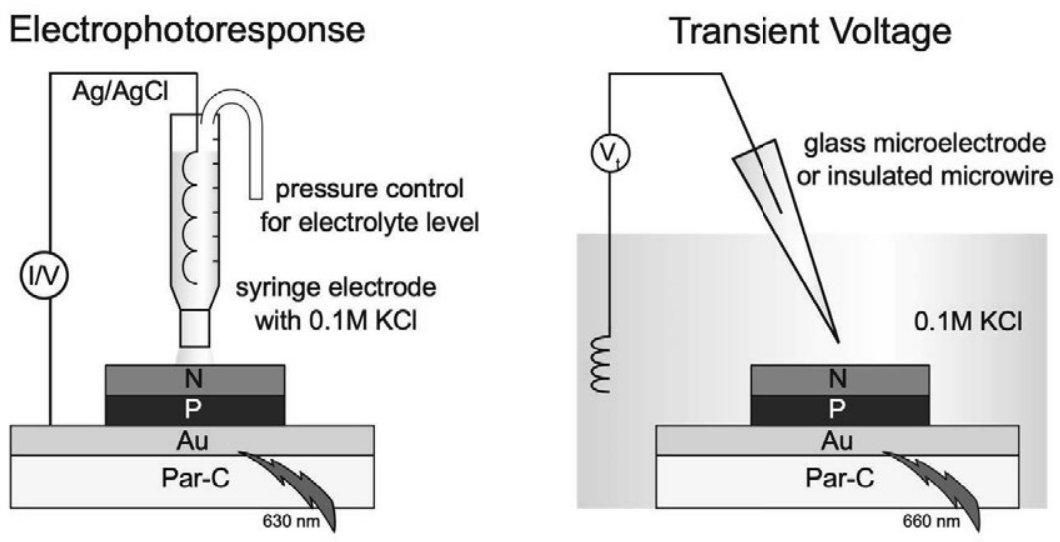

Figure 6.10: The electrical configuration for an electrophotoresponse and transient voltage measurement. 


\subsubsection{Transient voltage}

Even though EPR is a very good technique to select for promising device concepts, it does not reflect how the OEPC device behaves in the final application, where it is electrically floating. In order to study the real-life behavior, the voltage perturbation - i.e. transient voltage above the illuminated photopixel - is measured. This can be achieved by placing a glass microelectrode above the surface of the pixel and a reference electrode far away from the device as reported previously ${ }^{7}$ or by using an insulated microwire in place of the glass microelectrode (Figure 6.10). In order to be able to compare samples, the microelectrode distance from the sample should be kept constant. In case of Paper I, the voltagefollower electrode inserted in a Xenopus laevis oocyte was used for the measurement, and since most oocytes are similar in size, the cells themselves served as the height control. In Paper IV, the transient voltage produced by the photovoltaic microstimulator was measured by placing an insulated $\mathrm{Ag}$ wire above the microelectrodes.

\subsubsection{Electrophysiology recordings}

Electrophysiology is a branch of physiology concerned with the study of ionic currents in cells and tissue. It spans from single proteins (ion channels) to full organs. The techniques to measure the electrical behavior are either intracellular (single cell studies) or extracellular (single cells up to organ scale). Intracellular recordings offer large signal amplitude and high signal-to-noise ratio and are ideal for ion channel or individual cell studies. Typically, the intracellular techniques (e.g. voltage clamp) are invasive and are not applicable for long term studies. Extracellular techniques (e.g. multielectrode arrays, MEAs) are ideal for large throughput, complex, or long-term recordings. Some of the most popular platforms to record, and/or stimulate with extracellular electrodes are the integrated open-source amplifier circuits produced by Intan Technologies.

\subsubsection{Voltage clamp}

Voltage clamp is the most employed technique in the study of ion channels. In the electrophysiology dictionary, to "clamp" means to control. In voltage clamp, the membrane potential is set to a certain value and the current necessary for keeping the potential constant is measured. The recorded current is the same in magnitude and opposite in sign to the ionic current passing through the membrane. Therefore, by measuring the 
membrane current at different potentials, the ion channel voltage characteristic can be determined (Figure 6.11). The conductance curve can be calculated according to Ohm's law by the equation:

$$
G(V)=\frac{I}{\left(V-V_{\text {rev }}\right)}
$$

Where $G$ is the channel conductance, $I$ the steady state ionic current, $V$ the absolute membrane potential, and $V_{\text {rev }}$ the reversal potential $(-80 \mathrm{mV}$ for Shaker $K_{V}$ channels).

The two most-used configurations are two-electrode voltage clamp and patch clamp. The two-electrode voltage clamp setup consists of two glass intracellular microelectrodes. The voltage-measuring electrode records the voltage via a high-impedance follower circuit. The measured potential is compared to the command voltage, and in case of a difference, compensating current is delivered through the current-supplying electrode by a negative feedback amplifier (Figure 6.11). ${ }^{159}$ In patch clamp, a single microelectrode in contact with the cell membrane is used to measure the voltage and supply the current. This technique is only applicable in cases when small currents are passing through the membrane (small cells or a part of a membrane), since large currents would alter the set voltage significantly. In this thesis, the two-electrode voltage clamp was applied to study light-induced shift in the voltage characteristic of the Shaker voltage-gated potassium channel using Xenopus laevis oocytes as the in vitro model. The resulting conductance curves were fitted with the Boltzmann equation raised to the $4^{\text {th }}$ power and the voltage shift was calculated at $10 \%$ of the control maximum conductance. ${ }^{160}$

$$
G(V)=\frac{A}{\left(1+e^{\frac{V_{50}-V}{S}}\right)^{4}}
$$

Where $A$ is the amplitude, $V_{50}$ the midpoint, and $S$ the slope factor. 

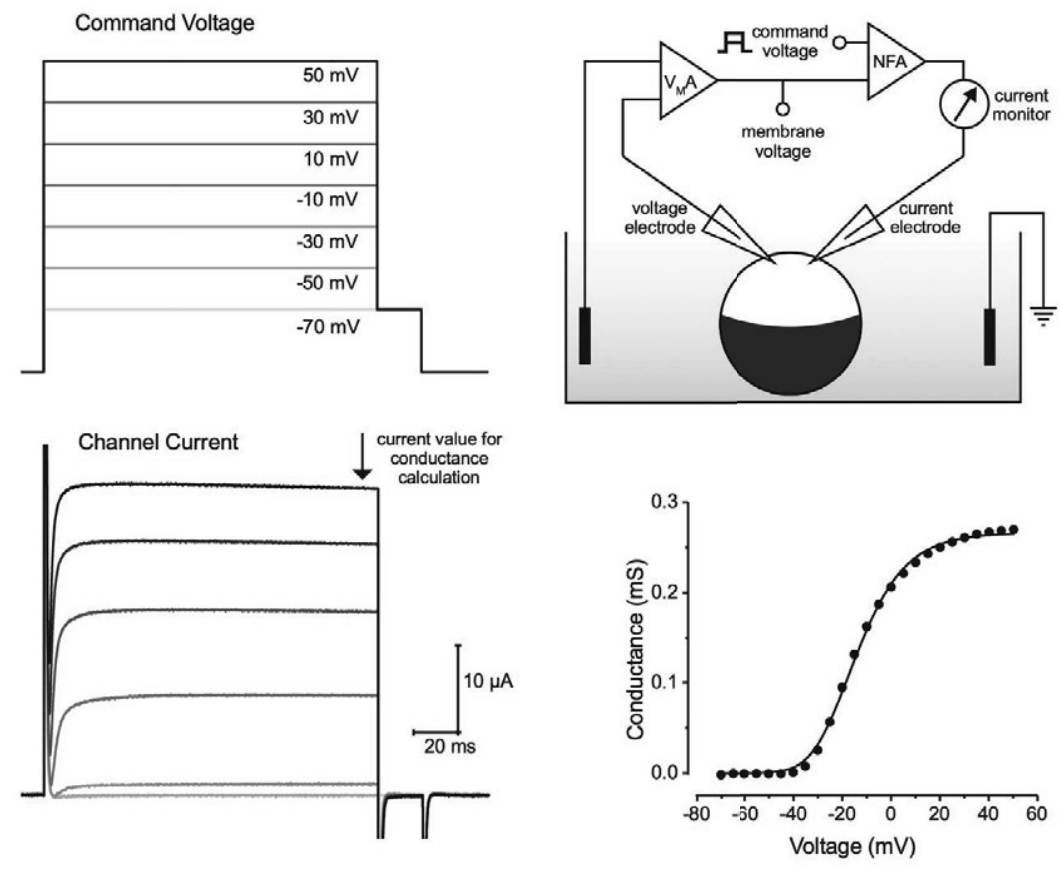

Figure 6.11: The two-electrode voltage clamp scheme along with a typical experiment demonstrated on the Shaker $\mathrm{K}_{\mathrm{V}}$ channel. $\mathrm{V}_{\mathrm{M}} \mathrm{A}$ : membrane voltage amplifier, NFA: negative feedback amplifier.

\subsubsection{Biopotential measurements using the Intan platform}

The Intan Technologies integrated circuits for extracellular recording consist of two main parts. The recording headstage amplifies, filters, and digitizes the signals from the connected electrodes. The recording controller or USB interface board links the headstages and other analog or digital signals with the computer. An Intan RHD USB interface board was used for validation of the sciatic nerve stimulation in rats. Tungsten microwires inserted in the muscles or high area gel electrodes placed on the hind leg surface were connected through a custom-built amplifier board to the analog input on the Intan interface board and were used to record the compound muscle action potentials (CMAPs) in the muscles innervated by the sciatic nerve. The data processing was done using Python or MATLAB. 


\subsubsection{Scanning electron microscopy}

Scanning electron microscopy (SEM) is an imaging technique based on specimen scanning by an electron beam. It offers resolution down to $1 \mathrm{~nm}$ in ideal conditions. One of the greatest strengths of SEM is the large depth of focus allowing for specimens of complex vertical structures to be imaged in focus. High vacuum is necessary for high resolution imaging. Low vacuum, "environmental" SEM techniques are an alternative for sensitive samples. The electron source can be based on thermionic or high field emission. High tension guns are more robust, but require ultrahigh vacuum for operation. The energy of the electron beam is typically in the range of $0.5-40 \mathrm{keV}$. The electron beam interacts with the observed species giving rise to a number of signals carrying information about the physical and chemical properties of the specimen. The interaction volume depends on the acceleration voltage, specimen atomic number, and the electron beam incidence angle. The signals come from different parts of the interaction volume based on the physical phenomenon causing them, including secondary electrons, backscattered electrons, Auger electrons, X-rays, cathodoluminescence, etc. (Figure 6.12). The escape depth of the signal dictates its resolution. ${ }^{161}$ The lowest energy Auger electrons have the shortest escape depth thus providing the best resolution. However, because the number of emitted Auger electrons is very low, the imaging takes longer time. That is why secondary electron (SE) detectors are the most common in SEM. They provide very good resolution due to the limited escape depth and strong signal because of the SE abundance. The in-lens detector collects secondary electrons ejected in a direction normal to the substrate plane showing minimal scattering, thus providing high-resolution images of the surface of the sample. Additional contrast is given by different yield of secondary electrons from different elements. On the other hand, an SE2 detector collects secondary electrons from deeper planes of the interaction volume with higher level of scattering, making it better for resolving 3-D topography. Therefore, the SE2 detector has lower resolution, but is less affected by sample charging, a problem in specimens not in perfect electrical contact with the stage. Thin samples of semiconductors or insulators can be imaged at low acceleration voltages when deposited on a conductive substrate, while thicker samples have to be coated with a thin film of metal. Typical acceleration voltage for imaging thin films of organic semiconductors is $\sim 3 \mathrm{kV}$. SEM was used to study the 
morphology, possible recrystallization, and microdelamination of the OEPC samples.

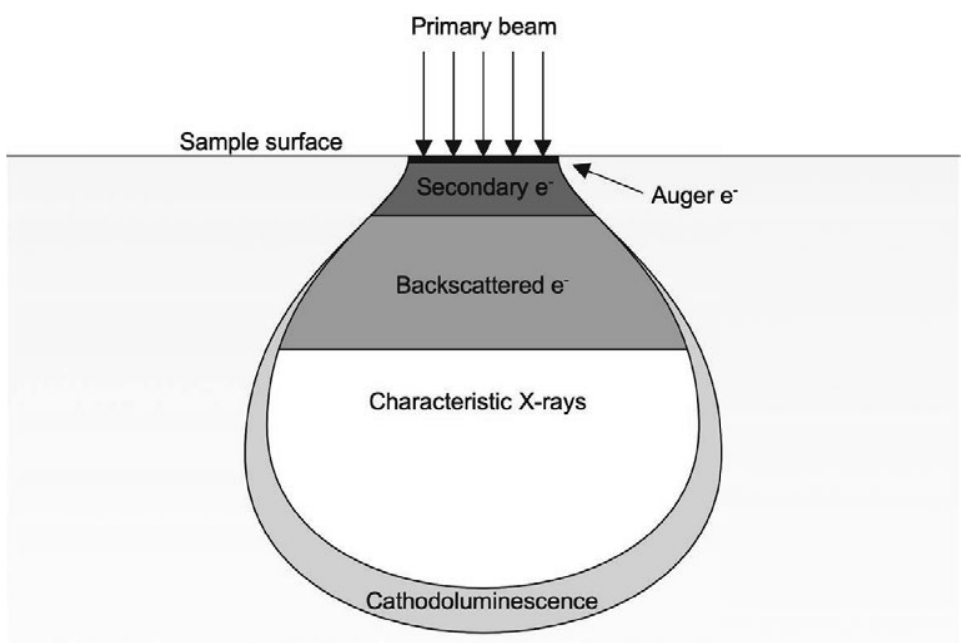

Figure 6.12: The interaction volume of primary electron beam with the specimen with highlighted regions of the signal origin.

\subsubsection{Fluorescence microscopy}

Fluorescence microscopy is a type of optical microscopy that uses fluorescence or phosphorescence for image formation. The sample fluorophores are excited with a specific wavelength chosen by an optical excitation filter. The resulting fluorescence light of lower energy is separated from the reflected excitation light by a dichroic mirror and an emission filter before reaching the detector. This means that each fluorophore requires a specific set of filters. The excitation wavelength is given by the energy difference between the fluorophore highest occupied molecular orbital and the lowest unoccupied molecular orbital, or by the bandgap in case of semiconductors. Semiconductor fluorescence intensity is very sensitive to surface states and defects; therefore, it can be used to study their integrity. Fluorescence microscopy was used to assess thin films of epindolidione patterned with the new lithography technique introduced in Paper II in comparison to continuous films. 


\subsubsection{Light sources}

A variety of red light sources were used throughout the thesis. The electrophotoresponse and OPV characterization was done with a $3 \mathrm{~W}$ electrical power LED $(630 \mathrm{~nm})$. All OEIP characterization and long-term illumination studies were done with an optical diffuser to ensure homogeneous light intensity over the cells connected in series. A highdensity LED array strobe light (626 nm) was used for photodriven ion delivery experiments (continuous, diffuser, $2.48 \mathrm{~mW} / \mathrm{cm}^{2}$ ) and for long term OEPC stability studies (pulsed, $8 \mathrm{~mW} / \mathrm{cm}^{2}, 20 \mathrm{~ms}$ ON, $550 \mathrm{~ms}$ OFF). A Thorlabs $(660 \mathrm{~nm})$ high power LED was used in the in vitro and in vivo experiments. The chronic in vivo experiments in Paper III were performed using a $638 \mathrm{~nm}$ laser diode of various power equipped with beam shaping optics. A calibrated Thorlabs Si p-i-n diode (Thorlabs SM1PD1B) was used to measure the light source intensities. Thorlabs LED Driver for High-Power LEDs (DC2200) was used in most of the pulsed light applications. 


\section{Conclusion}

The devices and concepts presented in this thesis are a result of my lasting interest in photostimulation of excitable tissue. Towards the end of my MSc studies we developed a novel device, the organic electrolytic photocapacitor, capable of direct photoelectrical stimulation of explanted retinas and cultured neural cells. ${ }^{7}$ In the original invention we introduced a very simple concept based on organic bilayer photovoltaics that allowed direct interfacing to the tissue without the need for encapsulation. Such an approach is typically not possible in case of inorganic devices. The obvious next step was to study the stimulation mechanism on a simpler, well-defined model, single non-excitable cells with overexpressed ion channels. We designed these experiments to conclusively establish that photocapacitive coupling was the stimulation mechanism. In Paper I, we used Xenopus laevis oocytes, which allowed us to investigate specific ion channel behavior in response to OEPC stimulation with the voltageclamp technique. By studying voltage-gated ion channel conductance characteristics, we could show that the photocapacitors were effectively depolarizing the cells by $\sim 20-30 \mathrm{mV}$ when illuminated with pulsed light. During the study we discounted the possibility of thermal effects by showing that device alternatives producing purely heat instead of photovoltage showed no effect on the channel behavior. Similarly, we discussed how changing the return electrode material can switch the working mechanism from photocapacitive to photofaradaic. Furthermore, the photocapacitive devices showed promising stability during 27 million charge/discharge cycles.

The next challenge was finding a method to pattern the semiconductors with better resolution. Organic, small-molecule semiconductors are typically processed by thermal evaporation. Due to the sensitivity of the materials, shadow masking is the predominant patterning technique. However, shadow masking limits the resolution to tens of micrometers, does not allow arbitrary designs, and is not suitable for multilayer structures of small sizes. Therefore, in Paper II we show a lithographybased technique applicable to a number of organic electronic materials. By using double UV-exposure for a positive tone photoresist, we allow for the lift-off (or resist removal) to happen in basic aqueous solution compatible with the sensitive nature of organic semiconductors. With this 
technique we were able to produce organic pixels down to $10 \mu \mathrm{m}$ with vertical edges.

The next goal was to move the technology further and demonstrate its functionality in vivo. We decided to apply the photocapacitor to peripheral nervous system due to the ease of stimulation validation. In Paper III, in collaboration with Jennifer Gelinas and Dion Khodagholy, Columbia University, we show that the OEPC devices are able to chronically stimulate motor function in rat sciatic nerve, one of the most established models of the peripheral nerve system. These findings demonstrate that the implanted devices were delivering sufficient stimulation photocurrent to a depth of $10-15 \mathrm{~mm}$ when irradiated by a $638 \mathrm{~nm}$ laser. In order to be able to perform the in vivo experiments, the technology had to be transferred to a flexible, conformable substrate. Looking into the future translation, we chose an FDA approved medical implant material parylene $C$. The new substrate material presented new hurdles. Most importantly, finding a suitable material for a semitransparent bottom/return electrode was a challenge. Thin, $10 \mathrm{~nm}$ thick gold electrode produced the best performing devices, however, the stability of the ultrathin metal on parylene $\mathrm{C}$ was poor. Furthermore, the patterning method of Paper II was not compatible with the sensitive gold electrode. Therefore, a new patterning technique based on parylene peel-off lithography was developed to allow for lithographic patterning of the organic pixels. The secondary effect of the new patterning scheme was simultaneous protection of the bottom electrode by a layer of amorphous indium tin oxide. By combining this technology with a ziptie immobilization design, we produced a device that was readily implantable, wireless, and performing up to 3 months in vivo.

The results of Paper III were very promising, but the ultimate limitation of the photocapacitor technology is the necessary photo pixel size to deliver sufficient charge to stimulate deep under the skin. The absorber pixel size utilized in the sciatic nerve study was between 1 and $3 \mathrm{~mm}$ thus providing little or no specificity. In order to achieve more efficient and specific devices, we decided to decouple the charge generation and stimulation into two moieties. The photoconversion is done by a photovoltaic cell placed subdermally to maximize effective light utilization, while the stimulation is performed by a pair of microelectrodes placed in the target location connected to the photovoltaics by a flexible lead. This concept was born during the initial 
in vivo experiments on sciatic nerve at Columbia University with an idea of producing a wireless stimulation depth brain probe for epilepsy studies. The fabrication method to produce such monolithic devices with parameters matching an application in rat hippocampus is the content of Paper IV.

During the work on organic electrolytic photocapacitor devices, it became clear that the organic semiconductor stack was very stable in physiological solutions. Therefore, an idea to utilize the materials in true photovoltaic cells for powering of other implantable technology was born. In Paper V, we decided to combine the photovoltaic driver with another thin film organic biomedical device originating from our group, the electrophoretic drug delivery system called organic electronic ion pump. The result is a monolithic drug delivery device controlled by light. The drug release is dictated by the applied light dose, which can be tuned by the light intensity or illumination time. This technology could be highly enabling in subcutaneous applications, especially where ultraprecise dosing is not necessary (e.g. chemotherapy).

\subsection{Future outlook}

The thesis is built around the applications of an organic bilayer heterojunction to stimulation and power conversion devices. It should be said that within the original photocapacitor work preceding this thesis, significant effort was put into finding the optimal semiconductor duo. The materials used were chosen based on the bilayer stability and device efficiency. From the insights learned within the course of this thesis, the stability of the heterojunction itself is excellent. The cause of device failures or instabilities is either the electrode-organic interface or the electrodes themselves. Further optimization of the interfaces is necessary to improve the device efficiency and in vivo stability.

The device efficiency could be improved by several approaches. Employing concepts such as vertical tandems (as shown in Paper V), increases the photovoltage and thus device efficiency per area. Translating the tandem technology to the photocapacitor devices would allow miniaturization of the photopixel area. We applied this approach and could see a significant enhancement of the stimulation efficiency, however, the open tandem stack proved to be instable in physiologic conditions. Therefore, further optimization of the tandem recombination 
layer would be necessary. The electrolytic capacitance of the organic heterojunction is relatively low and thus high-capacitance coatings can also increase the photocapacitor efficiency. One of the publications not included in the scope of the thesis describes this effect on the example of PEDOT:PSS coating. ${ }^{162}$ A different approach to increase the interfacial capacitance would be to cover the heterojunction with metal nanoparticles. Another major aspect related to the device efficiency in vivo is the wavelength at which it is operated. The absorber used in this thesis operates well in the $620-650 \mathrm{~nm}$ part of the tissue transparency window. However, the tissue is more transparent at longer wavelengths (for instance around $700 \mathrm{~nm}$ ), thus allowing for higher light coupling efficiency. By finding material combinations operating optimally at longer wavelengths, the devices could be either miniaturized or used even deeper inside of the tissue.

The photocapacitor stability is limited mostly by delamination of the electrode material from the substrate or the heterojunction from the electrode. Improved stability in vivo could be achieved by moving from the fully "open" device concept. For example, coating the full structure with a stable, high dielectric constant material would act as an extra protection layer while still providing high efficiency capacitive coupling to the tissue. Since delamination typically occurs from the material edges, their selective encapsulation could also lead to more stable devices. In case of the fully encapsulated photovoltaic devices, the source of instability is different. We suspect the titanium top electrode to be the cause of increasing serial resistance of the devices when exposed to physiological conditions. Therefore, optimization of the electrode materials and/ or of the encapsulation layers could provide better stability in aqueous environments.

Selectivity is another characteristic of the photocapacitive devices that should be investigated. One approach is introduced in Paper IV, where the stimulation moiety is miniaturized and detached from the charge generation component. A different approach would be to provide a structure to concentrate the charge produced by the photocapacitor device to a smaller area. This could be potentially achieved by introducing a charge collection layer on top of the photopixel and its partial encapsulation. However, this approach is complicated by the sensitive nature of the organic material and its incompatibility with further processing. 
Finally, it is worth mentioning that organic photovoltaic materials were used in this thesis to provide ultrathin, flexible devices not afforded by their rigid inorganic counterparts. However, when thickness and rigidity are not an issue, using inorganic photovoltaics provides significantly higher power conversion efficiencies. Another aspect is, that the world of organic electronics also offers polymeric materials with higher efficiencies than the small molecule semiconductors employed in this thesis, however, most of those show poor stability in aqueous environment. Furthermore, a bilayer heterojunction is necessary for the operation mechanism of the photocapacitor devices. The photogenerated charges have to be spatially separated between the photopixel and the return electrode. Bulk heterojunctions would necessitate an additional charge-selective layer on top of the photopixel to achieve the separation thus making the device more complex, which when combined with the limited stability makes them less ideal for the "open" architecture of the traditional photocapacitor. On the other hand, the photovoltaic cells utilized in Paper IV and V are fully encapsulated and thus using organic photovoltaic materials based on polymers could achieve higher efficiencies while keeping the superior material properties over inorganics. $^{124}$ 



\section{References}

1. Shirakawa, H., Louis, E. J., MacDiarmid, A. G., Chiang, C. K. \& Heeger, A. J. Synthesis of Electrically Conducting Organic Polymers : J. Chem. Soc. Chem. Commun. 578-580 (1977).

2. Tang, C. W. \& VanSlyke, S. A. Organic electroluminescent diodes. Appl. Phys. Lett. 51, 913-915 (1987).

3. Tang, W. Two-layer organic photovoltaic cell. Appl. Phys. Lett. 14650, 19851987 (1986).

4. Simon, D. T., Gabrielsson, E. O., Tybrandt, K. \& Berggren, M. Organic Bioelectronics: Bridging the Signaling Gap between Biology and Technology. Chem. Rev. 116, 13009-13041 (2016).

5. Lee, J., Jang, J. \& Song, Y. A Review on Wireless Powering Schemes for Implantable Microsystems in Neural Engineering Applications. Biomed. Eng. Lett. 6, 205-215 (2016).

6. Wangatia, L. M., Yang, S., Zabihi, F., Zhu, M. \& Ramakrishna, S. Biomedical electronics powered by solar cells. Curr. Opin. Biomed. Eng. 13, 25-31 (2019).

7. Rand, D. et al. Direct Electrical Neurostimulation with Organic Pigment Photocapacitors. Adv. Mater. 30, 1707292 (2018).

8. Isaksson, J. et al. Electronic control of $\mathrm{Ca} 2+$ signalling in neuronal cells using an organic electronic ion pump. Nat. Mater. 6, 673-679 (2007).

9. Hoppe, H. \& Sariciftci, N. S. Organic solar cells : An overview. J. Mater. Res. 19, 1924-1945 (2004).

10. Peumans, P., Yakimov, A. \& Forrest, S. R. Small molecular weight organic thin-film photodetectors and solar cells. J. Appl. Phys. 93, 3693-3723 (2003).

11. Green, M. A. et al. Solar cell efficiency tables (version 56). Prog. photovoltaics 2, 629-638 (2020).

12. Peumans, P., Uchida, S. \& Forrest, S. R. Efficient bulk heterojunction photovoltaic cells using small- molecular-weight organic thin films. Nature 425, 158-162 (2003).

13. Hiramoto, M., Suezaki, M. \& Yokoyama, M. Effect of Thin Gold Interstitiallayer on the Photovoltaic Properties of Tandem Organic Solar Cell. Chem. Lett. 19, 327-330 (1990).

14. Inganäs, O. Organic Photovoltaics over Three Decades. Adv. Mater. 30, 1800388-1800388 (2018).

15. From Molecules to Networks: An Introduction to Cellular and Molecular Neuroscience. (Elsevier, 2014).

16. Cole, B. Y. K. S. \& Curtis, H. J. Electric impedance of the squid giant axon during activity. J. Gen. Physiol. 22, 649-670 (1939).

17. Hodgkin, A. L., Huxley, A. F. \& Katz, B. Measurement of current-voltage relations in the membrane of the giant axon of Loligo. J. Physiol. 116, 424448 (1952).

18. Hodgkin, A. L. \& Huxley, A. F. Currents carried by sodium and potassium ions through the membrane of the giant axon of Loligo. J. Physiol. 116, 449472 (1952). 
19. Hodgkin, A. L. \& Huxley, A. F. The components of membrane conductance in the giant axon of Loligo. J. Physiol. 116, 473-496 (1952).

20. Hodgkin, A. L. \& Huxley, A. F. The dual effect of membrane potential on sodium conductance in the giant axon of Loligo. J. Physiol. 116, 497-506 (1952).

21. Hodgkin, A. L. \& Huxley, A. F. A quantitative description of membrane current and its application to conduction and excitation in nerve. J. Physiol. 117, 500-544 (1952).

22. Rosenbaum, T. \& Simon, S. A. TRPV1 receptors and signal transduction. in TRP ion channel function in sensory transduction and cellular signalling cascades (eds. Liedtke, W. B. \& Heller, S.) 69-84 (CRC Press, 2007).

23. Catsch, A. Eine erbliche Storung des Bewegungsmechanismus bei Drosophila melanogaster. Z. Indukt. Abstamm. Vererbungsl. 82, 64-66 (1944).

24. Papazian, D. M., Schwarz, T. L., Tempel, B. L., Jan, Y. U. H. N. \& Jan, L. Y. Cloning of Genomic and Complementary DNA from Shaker, a Putative Potassium Channel Gene from Drosophila. Science 237, 749-753 (1987).

25. Toshinori Hoshi, William N. Zagotta, R. W. A. Biophysical and Moleculr Mechanisms of Shaker Potassium Channel Inactivation. Science 250, 533-538 (1990).

26. Ottosson, N. E., Liin, S. I. \& Elinder, F. Drug-induced ion channel opening tuned by the voltage sensor charge profile. J. Gen. Physiol. 143, 173-82 (2014).

27. Gurdon, J. B., Lane, C. D., Woodland, H. R. \& Marbaix, G. Use of frog eggs and oocytes for the study of messenger RNA and its translation in living cells. Nature 233, 177-182 (1971).

28. Felten, D. L., O’Banion, M. K. \& Maida, M. S. Peripheral Nervous System. in Netter's Atlas of Neuroscience 153-231 (Elsevier, 2016).

29. Peltonen, S., Alanne, M. \& Peltonen, J. Barriers of the peripheral nerve. Tissue Barriers 1, e24956 (2013).

30. Neuroscientific Foundations of Anesthesiology. (Oxford University Press, 2011).

31. Swett, J. E., Wikholm, R. P., Blanks, R. H. I., Swett, A. L. \& Conley, L. C. Motoneurons of the Rat Sciatic Nerve. Exp. Neurol. 93, 227-252 (1986).

32. Vela, F. J. et al. Animal models used to study direct peripheral nerve repair: a systematic review. Neural Regen. Res. 15, 491-502 (2020).

33. Asato, F., Butler, M., Blomberg, H. \& Gordh, T. Variation in rat sciatic nerve anatomy : Implications for a rat model of neuropathic pain. J. Peripher. Nerv. Syst. 5, 19-21 (2000).

34. Schmalbruch, H. Fiber Composition of the Rat Sciatic Nerve. Anat. Rec. 215, 71-81 (1986).

35. Larson, C. E. \& Meng, E. A review for the peripheral nerve interface designer. J. Neurosci. Methods 332, 108523 (2020).

36. Varejão, A. S. P., Meek, M. F., Ferreira, A. J. A., Patrício, J. A. B. \& Cabrita, A. M. S. Functional evaluation of peripheral nerve regeneration in the rat: walking track analysis. J. Neurosci. Methods 108, 1-9 (2001). 
37. Azarmanesh, M. et al. Passive microinjection within high-throughput microfluidics for controlled actuation of droplets and cells. Sci. Rep. 9, 6723 (2019).

38. Sjöström, T. A. et al. Miniaturized Ionic Polarization Diodes for Neurotransmitter Release at Synaptic Speeds. Adv. Mater. Technol. 5, 1900750 (2019).

39. Vadlapatla, R., Wong, E. Y. \& Gayakwad, S. G. Electronic drug delivery systems: An overview. J. Drug Deliv. Sci. Technol. 41, 359-366 (2017).

40. Laser, D. J. \& Santiago, J. G. A review of micropumps. J. Micromechanics Microengineering 14, R35-R64 (2004).

41. Donahue, M. J., Proctor, C. \& Strakosas, X. Polymers / PEDOT Derivatives for Bioelectronics. in Redox Polymers for Energy and Nanomedicine 488-545 (2021). doi:10.1039/9781788019743-00488

42. Svirskis, D., Travas-sejdic, J., Rodgers, A. \& Garg, S. Electrochemically controlled drug delivery based on intrinsically conducting polymers. $J$. Control. Release 146, 6-15 (2010).

43. Simon, D. T. et al. Organic electronics for precise delivery of neurotransmitters to modulate mammalian sensory function. Nat. Mater. 8 , 742-746 (2009).

44. Jonsson, A. et al. Therapy using implanted organic bioelectronics. Sci. Adv. 1, e1500039 (2015).

45. Proctor, C. M. et al. Electrophoretic drug delivery for seizure control. Sci. Adv. 4, eaau1291 (2018).

46. Schoen, I. \& Fromherz, P. The mechanism of extracellular stimulation of nerve cells on an electrolyte-oxide-semiconductor capacitor. Biophys. J. 92, 1096-1111 (2007).

47. Merrill, D. R., Bikson, M. \& Jefferys, J. G. R. Electrical stimulation of excitable tissue : design of efficacious and safe protocols. J. Neurosci. Methods 141, 171-198 (2005).

48. Cogan, S. F. et al. Tissue damage thresholds during therapeutic electrical stimulation. J. Neural Eng. 13, (2016).

49. Cogan, S. F., Hara, S. \& Ludwig, K. A. The Safe Delivery of Electrical Currents and Neuromodulation. in Neuromodulation 83-94 (Elsevier Ltd, 2018). doi:10.1016/B978-0-12-805353-9.00007-3

50. Cogan, S. F. Neural Stimulation and Recording Electrodes. Annu. Rev. Biomed. Eng. 10, 275-309 (2008).

51. Kim, Y. H., Kim, G. H., Kim, A. Y. \& Han, Y. H. In vitro extracellular recording and stimulation performance of nanoporous gold-modified multielectrode arrays. J. Neural Eng. 12, 66029 (2015).

52. Fan, B., Rodriguez, A. V, Vercosa, D. G., Kemere, C. \& Robinson, J. T. Sputtered porous $\mathrm{Pt}$ for wafer-scale manufacture of low-impedance flexible microelectrodes. J. Neural Eng. 17, 036029 (2020).

53. Boehler, C., Vieira, D. M., Egert, U. \& Asplund, M. NanoPt - A Nanostructured Electrode Coating for Neural Recording and Microstimulation. ACS Appl. Mater. Interfaces 12, 14855-14865 (2020).

54. Rivnay, J. et al. High-performance transistors for bioelectronics through tuning of channel thickness. Sci. Adv. 1, e1400251 (2015). 
55. Cui, X. \& Martin, D. C. Electrochemical deposition and characterization of poly(3,4-ethylenedioxythiophene) on neural microelectrode arrays. Sensors Actuators B 89, 92-102 (2003).

56. Boehler, C., Oberueber, F., Schlabach, S., Stieglitz, T. \& Asplund, M. LongTerm Stable Adhesion for Conducting Polymers in Biomedical Applications: IrOx and Nanostructured Platinum Solve the Chronic Challenge. Appl. Mater. Interfaces 9, 189-197 (2017).

57. Pranti, A. S., Schander, A., Bödecker, A. \& Lang, W. PEDOT: PSS Coating on Gold Microelectrodes with Excellent Stability and High Charge Injection Capacity for Chronic Neural Interfaces. Sensors Actuators B 275, 382-393 (2018).

58. Mitraka, E. et al. Electrocatalytic Production of Hydrogen Peroxide with Poly(3,4-ethylenedioxythiophene) Electrodes. Adv. Sustain. Syst. 3, 1800110 (2018).

59. Jiang, Y. et al. Nongenetic optical neuromodulation with silicon-based materials. Nat. Protoc. 14, 1339-1376 (2019).

60. Cotero, V. et al. Noninvasive sub-organ ultrasound stimulation fortargeted neuromodulation. Nat. Commun. 10, 952 (2019).

61. Zachs, D. P. et al. Noninvasive ultrasound stimulation of the spleen to treat inflammatory arthritis. Nat. Commun. 10, 951 (2019).

62. Prieto, M. L., Firouzi, K., Khuri-Yakub, B. T., Madison, D. V. \& Maduke, M. Spike-frequency dependent inhibition and potentiation of neural activity by ultrasound. bioRxiv (2020). doi:https://doi.org/10.1101/2020.06.01.128710

63. Marino, A. et al. Piezoelectric Nanoparticle-Assisted Wireless Neuronal Stimulation. ACS Nano 9, 7678-7689 (2015).

64. Dobson, J. Remote control of cellular behaviour with magnetic nanoparticles. Nat. Nanotechnol. 3, 139-143 (2008).

65. Huang, H., Delikanli, S., Zeng, H., Ferkey, D. M. \& Pralle, A. Remote control of ion channels and neurons through magnetic-field heating of nanoparticles. Nat. Nanotechnol. 5, 602-606 (2010).

66. Chen, R., Romero, G., Christiansen, M. G., Mohr, A. \& Anikeeva, P. Wireless magnetothermal deep brain stimulation. Science 347, 1477-1480 (2015).

67. Đerek, V. et al. Untangling Photofaradaic and Photocapacitive Effects in Organic Optoelectronic Stimulation Devices. Front. Bioeng. Biotechnol. 8, $1-$ 8 (2020).

68. Ghezzi, D. et al. A hybrid bioorganic interface for neuronal photoactivation. Nat. Commun. 2, 166 (2011).

69. Ghezzi, D. et al. A polymer optoelectronic interface restores light sensitivity in blind rat retinas. Nat. Photonics 7, 400-406 (2013).

70. Mathieson, K. et al. Photovoltaic retinal prosthesis with high pixel density. Nat. Photonics 6, 391-397 (2012).

71. Maya-Vetencourt, J. F. et al. A fully organic retinal prosthesis restores vision in a rat model of degenerative blindness. Nat. Mater. 16, 681-689 (2017).

72. Palanker, D., Mer, Y. Le, Mohand-said, S., Muqit, M. \& Sahel, J. A. Photovoltaic Restoration of Central Vision in Atrophic Age-Related Macular Degeneration. Ophthalmology 127, 1097-1104 (2020). 
73. Smith, A. M., Mancini, M. C. \& Shuming, N. Second window for in vivo imaging n. Nat. Nanotechnol. 4, 710-711 (2009).

74. Bashkatov, A. N., Genina, E. A. \& Tuchin, V. V. Optical properties of skin, subcutaneous, and muscle tissues: a review. J. Innov. Opt. Health Sci. 4, 9-38 (2011).

75. Handbook of Low-Level Laser Therapy. (Pan Stanford Publishing Pte. Ltd., 2017).

76. American National Standard for Safe Use of Lasers. 76-77 (2007).

77. Tamura, T., Shamsuddin, A. K. M., Kawarada, A., Togawa, T. \& Oberg, P. A. Transcutaneous optical power converter for implantable devices. Proc. SPIE 2084, 99-104 (1993).

78. Hochbaum, D. R. et al. All-optical electrophysiology in mammalian neurons using engineered microbial rhodopsins. Nat. Methods 11, 825-833 (2014).

79. Gutruf, P. et al. Fully implantable optoelectronic systems for battery-free, multimodal operation in neuroscience research. Nat. Electron. 1, 652-660 (2018).

80. Shapiro, M. G., Homma, K., Villarreal, S., Richter, C.-P. \& Bezanilla, F. Infrared light excites cells by changing their electrical capacitance. Nat. Commun. 3, 736 (2012).

81. Albert, E. S. et al. TRPV4 channels mediate the infrared laser-evoked response in sensory neurons. J. Neurophysiol. 107, 3227-3234 (2012).

82. Carvalho-de-Souza, J. L. et al. Photosensitivity of neurons enabled by celltargeted gold nanoparticles. Neuron 86, 207-217 (2015).

83. Martino, N. et al. Photothermal cellular stimulation in functional bio-polymer interfaces. Sci. Rep. 5, 8911 (2015).

84. Sytnyk, M. et al. Cellular interfaces with hydrogen-bonded organic semiconductor hierarchical nanocrystals. Nat. Commun. 8, 91 (2017).

85. Jiang, Y. et al. Heterogeneous silicon mesostructures for lipid-supported bioelectric interfaces. Nat. Mater. 15, 1023-1030 (2016).

86. Gryszel, M. et al. General Observation of Photocatalytic Oxygen Reduction to Hydrogen Peroxide by Organic Semiconductor Thin Films and Colloidal Crystals. ACS Appl. Mater. Interfaces 10, 13253-13257 (2018).

87. Antognazza, M. R., Aziz, I. A. \& Lodola, F. Use of Exogenous and Endogenous Photomediators as Efficient ROS Modulation Tools: Results and Perspectives for Therapeutic Purposes. Oxid. Med. Cell. Longev. (2019).

88. Gamper, N. et al. Oxidative modification of M-type $\mathrm{K}+$ channels as a mechanism of cytoprotective neuronal silencing. EMBO J. 25, 4996-5004 (2006).

89. Velema, W. A., Szymanski, W. \& Feringa, B. L. Photopharmacology: Beyond proof of principle. J. Am. Chem. Soc. 136, 2178-2191 (2014).

90. Jiang, Y. et al. Rational design of silicon structures for optically controlled multiscale biointerfaces. Nat. Biomed. Eng. 2, 508-521 (2018).

91. Gryszel, M. \& Głowacki, E. D. Organic thin film photofaradaic pixels for ondemand electrochemistry in physiological conditions. Chem. Commun. 56, 1705-1708 (2020). 
92. Jakešová, M. et al. Optoelectronic control of single cells using organic photocapacitors. Sci. Adv. 5, eaav5265 (2019).

93. Olofsson, P. S. \& Bouton, C. Bioelectronic medicine: an unexpected path to new therapies. J. Intern. Med. 286, 237-239 (2019).

94. Cho, Y., Park, J., Lee, C. \& Lee, S. Recent progress on peripheral neural interface technology towards bioelectronic medicine. Bioelectron. Med. 6, (2020).

95. Neuroprosthetics: Theory and Practice. 8, (World Scientific Publishing, 2017).

96. Badia, J. et al. Comparative analysis of transverse intrafascicular multichannel, longitudinal intrafascicular and multipolar cuff electrodes for the selective stimulation of nerve fascicles. J. Neural Eng. 8, 036023 (2011).

97. Campbell, P. K. et al. A Silicon-Based, Three-Dimensional Neural Interface: Manufacturing Processes for an Intracortical Electrode Array. IEEE Trans. Biomed. Eng. 38, 758-768 (1991).

98. Jones, K. E., Campbell, P. K. \& Normann, R. A. A Glass / Silicon Composite Intracortical Electrode Array. Ann. Biomed. Eng. 20, 423-437 (1992).

99. Branner, A. \& Normann, R. A. A multielectrode array for intrafascicular recording and stimulation in sciatic nerve of cats. Brain Res. Bull. 51, 293-306 (2000).

100. Boretius, T. et al. A transverse intrafascicular multichannel electrode (TIME) to interface with the peripheral nerve. Biosens. Bioelectron. 26, 62-69 (2010).

101. Badia, J. et al. Biocompatibility of Chronically Implanted Transverse Intrafascicular Multichannel Electrode (TIME) in the Rat Sciatic Nerve. IEEE Trans. Biomed. Eng. 58, 2324-2332 (2011).

102. Deberge, L., Delleci, C., Joseph, P. A., Cassoudesalle, H. \& Glize, B. Longitudinal long-term evaluation of patients with a Brindley's neurostimulator and followed in Bordeaux university hospital: Return on 30 years of use. Ann. Phys. Rehabil. Med. 61, e380 (2018).

103. Waters, R. L., McNeal, D. R., Faloon, W. \& Clifford, B. Functional Electrical Stimulation of the Peroneal Nerve for Hemiplegia: Long-Term Clinical Follow-up. J. Bone Jt. Surg. 67, 792-793 (1985).

104. González-González, M. A. et al. Thin Film Multi-Electrode Softening Cuffs for Selective Neuromodulation. Sci. Rep. 8, 1-15 (2018).

105. Rodríguez, F. J. et al. Polyimide cuff electrodes for peripheral nerve stimulation. J. Neurosci. Methods 98, 105-118 (2000).

106. Lee, S. et al. Toward Bioelectronic Medicine - Neuromodulation of Small Peripheral Nerves Using Flexible Neural Clip. Adv. Sci. 4, 1700149 (2017).

107. Yu, H., Xiong, W., Zhang, H., Wang, W. \& Li, Z. A Parylene Self-Locking Cuff Electrode for Peripheral Nerve Stimulation and Recording. $J$. Microelectromechanical Syst. 23, 1025-1035 (2014).

108. Cobo, A. M. et al. Parylene-Based Cuff Electrode With Integrated Microfluidics for Peripheral Nerve Recording, Stimulation, and Drug Delivery. J. Microelectromechanical Syst. 28, 36-49 (2019).

109. Bettinger, C. J. Recent advances in materials and flexible electronics for peripheral nerve interfaces. Bioelectron. Med. 4, (2018). 
110. Pancrazio, J. J. \& Cogan, S. F. Editorial for the Special Issue on Neural Electrodes: Design and Applications. Micromachines 10, 466 (2019).

111. Agarwal, K., Jegadeesan, R., Guo, Y.-X. \& Thakor, N. V. Wireless Power Transfer Strategies for Implantable Bioelectronics: Methodological Review. IEEE Rev. Biomed. Eng. 10, 136-161 (2017).

112. Khan, S. R., Pavuluri, S. K., Cummins, G. \& Desmulliez, M. P. Y. Wireless Power Transfer Techniques for Implantable Medical Devices: A Review. Sensors 20, 3487 (2020).

113. Amar, A. Ben, Kouki, A. B. \& Cao, H. Power approaches for implantable medical devices. Sensors 15, 28889-28914 (2015).

114. Hernandez-Reynoso, A. G. et al. Miniature Electroparticle-Cuff for Wireless Peripheral Neuromodulation. J. Neural Eng. 16, 046002 (2019).

115. Khalifa, A. et al. The Microbead: A Highly Miniaturized Wirelessly Powered Implantable Neural Stimulating System. IEEE Trans. Biomed. Circuits Syst. 12, 521-531 (2018).

116. Piech, D. K. et al. A wireless millimetre-scale implantable neural stimulator with ultrasonically powered bidirectional communication. Nat. Biomed. Eng. 4, 207-222 (2020).

117. Song, Y. et al. A microscale photovoltaic neurostimulator for fiber optic delivery of functional electrical stimulation. J. Neural Eng. 4, 213-218 (2007).

118. Murakawa, K., Kobayashi, M., Nakamura, O. \& Kawata, S. A Wireless NearInfrared Energy System for Medical Implants. IEEE Eng. Med. Biol. 18, 7072 (1999).

119. Abdo, A. et al. Floating light-activated microelectrical stimulators tested in the rat spinal cord. J. Neural Eng. 8, 056012 (2011).

120. Kim, J. et al. Active photonic wireless power transfer into live tissues. PNAS 117, 16856-16863 (2020).

121. Song, K. et al. Subdermal Flexible Solar Cell Arrays for Powering Medical Electronic Implants. Adv. Healthc. Mater. 5, 1572-1580 (2016).

122. Goto, K., Nakagawa, T., Nakamura, O. \& Kawata, S. An Implantable Power Supply with an Optically Rechargeable Lithium Battery. IEEE Trans. Biomed. Eng. 48, 830-833 (2001).

123. Lu, L. et al. Biodegradable Monocrystalline Silicon Photovoltaic Microcells as Power Supplies for Transient Biomedical Implants. Adv. Energy Mater. 8, 1-8 (2018).

124. Park, S. et al. Self-powered ultra-flexible electronics via nano-gratingpatterned organic photovoltaics. Nature 561, 516-521 (2018).

125. Shi, B., Li, Z. \& Fan, Y. Implantable Energy-Harvesting Devices. Adv. Mater. 30, 1-18 (2018).

126. Hiramoto, M., Fujiwara, H. \& Yokoyama, M. Three-layered organic solar cell with a photoactive interlayer of codeposited pigments. Appl. Phys. Lett. 58, 1062-1064 (1991).

127. Photosensitisation: Molecular, Cellular and Medical Aspects. (SpringerVerlag, 1987).

128. Głowacki, E. D. et al. Epindolidiones - Versatile and Stable HydrogenBonded Pigments for Organic Field-Effect Transistors and Light-Emitting Diodes. Adv. Funct. Mater. 25, 776-787 (2014). 
129. Elschner, A., Kirchmeyer, S., Lövenich, W., Merker, U. \& Reuter, K. PEDOT: Principles and Applications of Intrinsically Conductive Polymer. (CRC Press, 2011).

130. Arbring Sjöström, T. et al. A crosslinked polyelectrolyte for improved selectivity and processability of iontronic systems. ACS Appl. Mater. Interfaces 9, 30247-30252 (2017).

131. Mattox, D. M. Handbook of Physical Vapor Deposition (PVD) Processing. (Elsevier, 2010).

132. Madorsky, S. L. Rates of Thermal Degradation of Polystyrene and Polyethylene in a Vacuum. J. Polym. Sci. IX, 133-156 (1951).

133. Forrest, S. R. Ultrathin Organic Films Grown by Organic Molecular Beam Deposition and Related Techniques. Chem. Rev. 2665, 1793-1896 (1997).

134. Franssila, S. Introduction to Microfabrication. (Wiley, 2004).

135. Fortin, J. B. \& Lu, T.-M. Chemical Vapor Polymerization, The Growth and Properties of Parylene Thin Films. (Springer US, 2004).

136. Ortigoza-diaz, J. et al. Techniques and Considerations in the Microfabrication of Parylene C Microelectromechanical Systems. Micromachines 9, 422 (2018).

137. Chang, J. H.-C., Lu, B. \& Tai, Y. C. Adhesion-enhancing surface treatments for parylene deposition. Proc. 16th Int. Solid-State Sens., Act. Microsyst. Conf. 390-393 (2011). doi:10.1109/TRANSDUCERS.2011.5969484

138. Lee, S. J. \& Kim, B. Deposition of silicon nitride film at room temperature using a SiH4 - NH3 - N2 plasma. J. Ceram. Soc. Japan 118, 1188-1191 (2010).

139. Metzler, M. \& Patel, R. Plasma Enhanced Chemical Vapor Deposition (PECVD) of Silicon Nitride (SiNx) Using Oxford Instruments System 100 PECVD. Univ. Pennsylvania Sch. (2017).

140. Glass, N. R., Tjeung, R., Chan, P. \& Yeo, L. Y. Organosilane deposition for microfluidic applications. Biomicrofluidics 5, 036501 (2011).

141. Stavrinidou, E. et al. Direct measurement of ion mobility in a conducting polymer. Adv. Mater. 25, 4488-4493 (2013).

142. Kurth, D. G. \& Bein, T. Monomolecular Layers and Thin Films. J. Phys. Chem. 96, 6707-6712 (1992).

143. Norrman, K., Ghanbari-Siahkali, A. \& Larsen, N. B. 6 Studies of spin-coated polymer films. Annu. Reports Sect. C Phys. Chem. 101, 174-201 (2005).

144. Scriven, L. E. Physics and Applications of Dip Coating and Spin Coating. MRS Symp. Proc. 121, 717-729 (1988).

145. Zakhidov, B. A. A. et al. Hydrofluoroethers as Orthogonal Solvents for the Chemical Processing of Organic Electronic Materials. Adv. Mater. 20, 34813484 (2008).

146. Zakhidov, A. A. et al. Orthogonal processing: A new strategy for organic electronics. Chem. Sci. 2, 1178-1182 (2011).

147. Kymissis, I. et al. Patterning pentacene organic thin film transistors Patterning pentacene organic thin film transistors. J. Vac. Sci. Technol. 20, 956-959 (2002). 
148. Tian, P. F. et al. Photolithographic patterning of vacuum-deposited organic light emitting devices. Appl. Phys. A Mater. Sci. Process. 71, 3197-3199 (1997).

149. DeFranco, J. A., Schmidt, B. S., Lipson, M. \& Malliaras, G. G. Photolithographic patterning of organic electronic materials. Org. Electron. 7, 22-28 (2006).

150. Shtein, M., Peumans, P., Benziger, J. B. \& Forrest, S. R. Micropatterning of small molecular weight organic semiconductor thin films using organic vapor phase deposition. J. Appl. Phys. 93, 4005-4016 (2003).

151. Decker, W., Belan, R., Heydemann, V. D., Armstrong, S. \& Fisher, T. Novel Low Pressure Sputtering Source and Improved Vacuum Deposition of Small Patterned Features Using Precision Shadow Masks. 59th Annu. Tech. Conf. Proc. 95-100 (2016). doi:10.14332/svc16.proc.0036

152. Mack, C. Fundamental Principles of Optical Lithography. (Wiley, 2007).

153. Menon, R., Patel, A., Gil, D. \& Smith, H. I. Maskless lithography. Mater. Today 8, 26-33 (2005).

154. Derek, V., Jakešová, M., Berggren, M., Simon, D. T. \& Głowacki, E. D. Micropatterning of organic electronic materials using a facile aqueous photolithographic process. AIP Adv. 8, 105116 (2018).

155. Ilic, B. \& Craighead, H. G. Topographical Patterning of Chemically Sensitive Biological Materials Using a Polymer-Based Dry Lift Off. Biomed. Microdevices 2, 317-322 (2000).

156. Cicoira, B. F. et al. Influence of Device Geometry on Sensor Characteristics of Planar Organic Electrochemical Transistors. Adv. Mater. 22, 1012-1016 (2010).

157. Jonsson, A. et al. Bioelectronic neural pixel: Chemical stimulation and electrical sensing at the same site. Proc. Natl. Acad. Sci. 113, 9440-9445 (2016).

158. Josephy, P. D., Eling, T. \& Mason, R. P. The Horseradish Peroxidasecatalyzed Oxidation of 3,5,3',5'- Tetramethylbenzidine. J. Biol. Chem. 257, 3669-3675 (1982).

159. Hille, B. Ion Channels of Excitable Membranes. (Sinauer Associates, Inc., 2001).

160. Börjesson, S. I., Parkkari, T., Hammarström, S. \& Elinder, F. Electrostatic tuning of cellular excitability. Biophys. J. 98, 396-403 (2010).

161. Vernon-Parry, K. D. Scanning Electron Microscopy: an introduction. III-VS Rev. 13, 40-44 (2000).

162. Ejneby, M. S. et al. Extracellular Photovoltage Clamp Using Conducting Polymer-Modified Organic Photocapacitors. Adv. Mater. Technol. 5, 1900860 (2020). 



\section{Included Publications}

All the included published papers are licensed under the Creative Commons Attribution license (CC BY 4.0):

http://creativecommons.org/licenses/by/4.0/ 



\section{Papers}

The papers associated with this thesis have been removed for copyright reasons. For more details about these see:

http://urn.kb.se/resolve?urn=urn:nbn:se:liu:diva-171846 


\section{FACULTY OF SCIENCE AND ENGINEERING}

Linköping Studies in Science and Technology, Dissertation No. 2105, 2021

Department of Science and Technology

Linköping University

SE-581 83 Linköping, Sweden

www.liu.se 